\title{
Virtual Optical Comparator
}

Federal Manufacturing \& Technologies

Greg Thompson

\section{KCP-613-8517}

Published October 2008

\section{Final Report}

Approved for public release; distribution is unlimited.

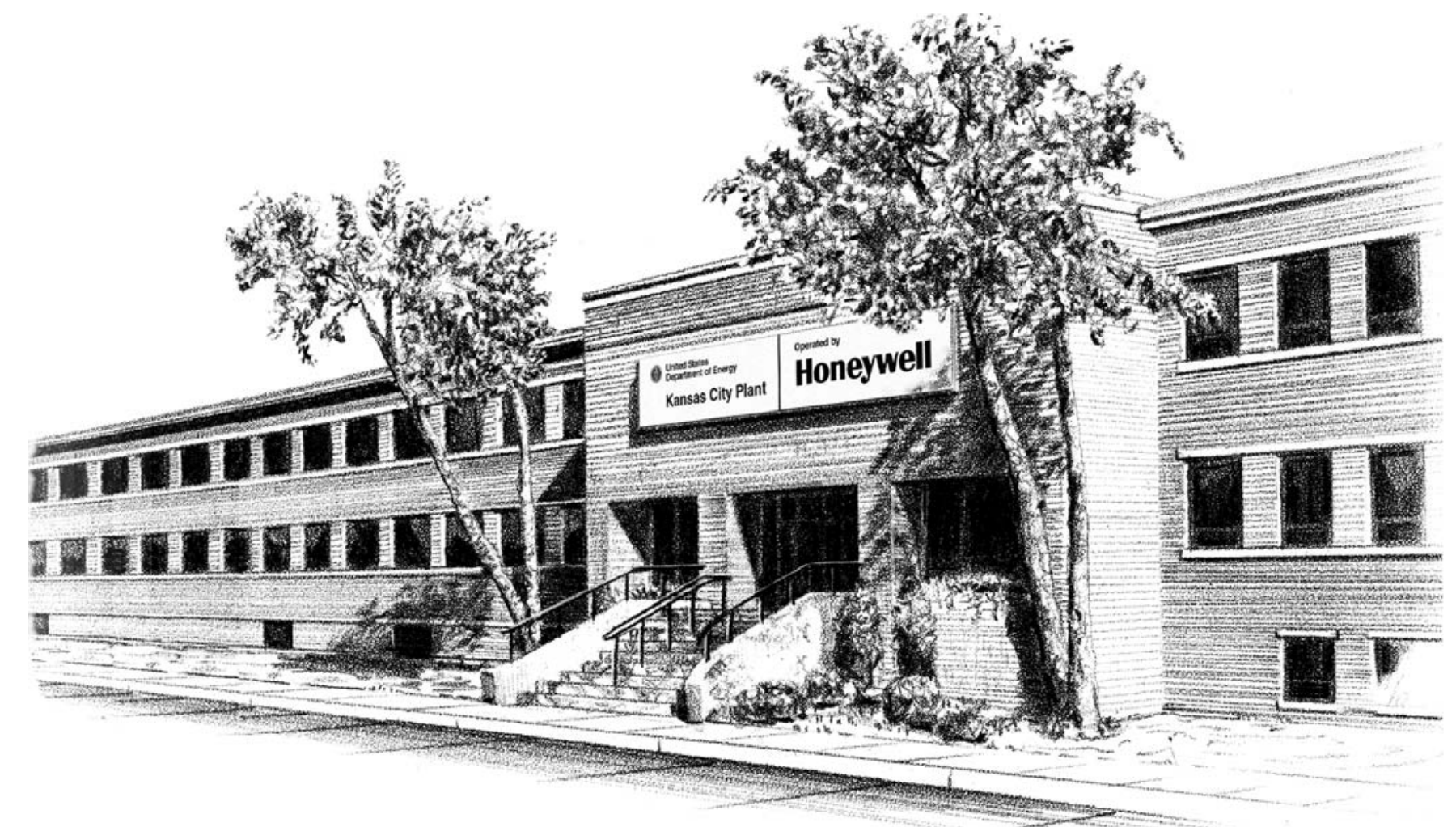

Prepared under prime contract DE-ACO4-01AL66850 for the United States Department of Energy 


\section{DISCLAIMER}

This report was prepared as an account of work sponsored by an agency of the United States Government. Neither the United States Government nor any agency thereof, nor any of their employees, makes any warranty, express or implied, or assumes any legal liability or responsibility for the accuracy, completeness, or usefulness of any information, apparatus, product, or process disclosed, or represents that its use would not infringe privately owned rights. Reference herein to any specific commercial product, process or service by trade names, trademark, manufacturer, or otherwise, does not necessarily constitute or imply its endorsement, recommendation or favoring by the United States Government or any agency thereof. The views and opinions of authors expressed herein do not necessarily state or reflect those of the United States Government or any agency thereof.

All data prepared, analyzed and presented has been developed in a specific context of work and was prepared for internal evaluation and use pursuant to that work authorized under the reference contract. Reference herein to any specific commercial product, process or service by trade name, trademark, manufacturer, or otherwise, does not necessarily constitute or imply its endorsement, recommendation or favoring by the United States Government, any agency thereof or Honeywell Federal Manufacturing \& Technologies, LLC.

Printed in the United States of America.

This report has been reproduced from the best available copy.

Available to DOE and DOE contractors from the Office of Scientific and Technical Information, P.O. Box 62, Oak Ridge, Tennessee 37831; prices available from (865) 576-8401, FTS 626-8401.

Available to the public from the National Technical Information Service, U.S. Department of Commerce, 5285 Port Royal, Rd., Springfield, Virginia 22161, (703) 487-4650.

A prime contractor with the United States Department of Energy under Contract Number DE-AC04-O1AL66850

\author{
Honeywell Federal Manufacturing \& Technologies \\ P.O. Box 419159 \\ Kansas City, Missouri, 64141-6159
}




\title{
Honeywell
}

\section{KCP-613-8517 \\ Distribution Category UC-42}

Approved for public release; distribution is unlimited.

\author{
Virtual Optical \\ Comparator \\ Greg Thompson \\ Published October 2008 \\ Final Report \\ Greg Thompson, Project Leader
}





\section{Contents}

Section Page

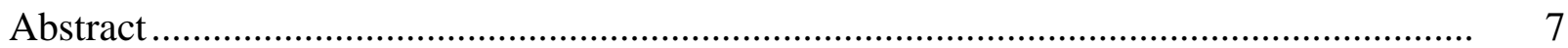

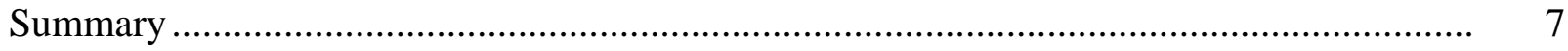

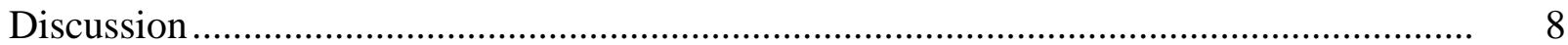

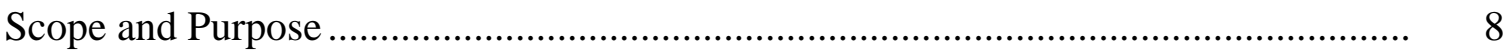

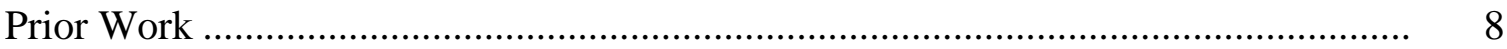

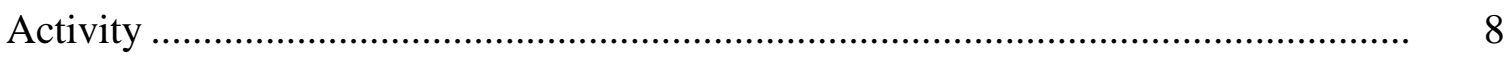

System Calibration.............................................................................. 18

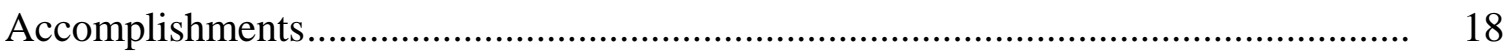

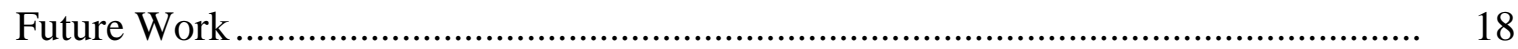

Appendices

A. Operating Manual .............................................................................. 19

B. Computer Source Code ...................................................................... 38 


\section{Illustrations}

Figure Page

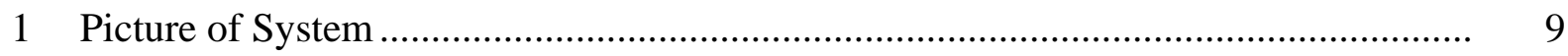

2 Traditional Optical Comparator ..................................................................... 10

3 Sample Part Drawing ................................................................................ 11

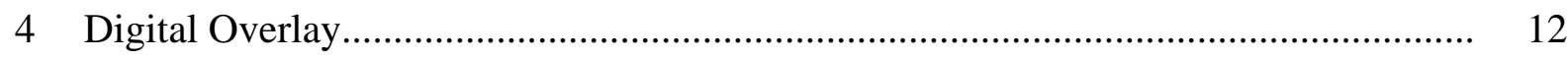

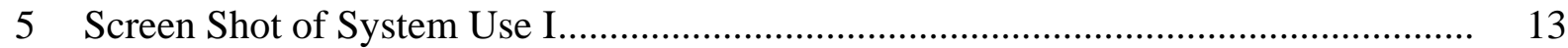

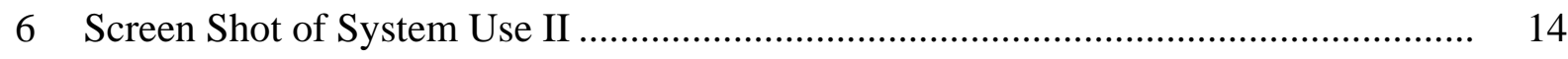

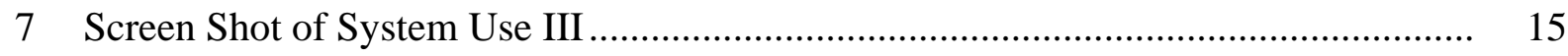

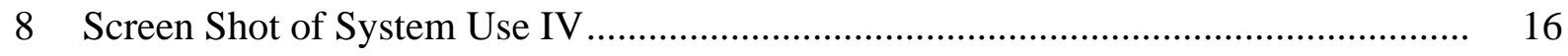

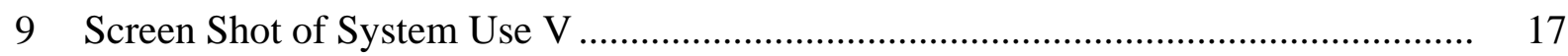




\begin{abstract}
The Virtual Optical Comparator, VOC, was conceived as a result of the limitations of conventional optical comparators and vision systems. Piece part designs for mechanisms have started to include precision features on the face of parts that must be viewed using a reflected image rather than a profile shadow. The VOC concept uses a computer generated overlay and a digital camera to measure features on a video screen. The advantage of this system is superior edge detection compared to traditional systems. No vinyl charts are procured or inspected. The part size and expensive fixtures are no longer a concern because of the range of the $X-Y$ table of the Virtual Optical Comparator. Product redesigns require only changes to the CAD image overlays; new vinyl charts are not required. The inspection process is more ergonomic by allowing the operator to view the part sitting at a desk rather than standing over a 30 inch screen. The procurement cost for the VOC will be less than a traditional comparator with a much smaller footprint with less maintenance and energy requirements.
\end{abstract}

\title{
Summary
}

The Virtual Optical Comparator was conceived during the procurement of design gages for the W76-1 mechanisms. The WR-1 Dual Stronglink Mechanism has similar features. Piece part designs included many critical features that are machined on the face of a part. Traditionally these features are checked on optical comparators using reflected light to illuminate the part surfaces. The part tolerance required the use of at least $50 \mathrm{X}$ magnification. At this magnification the light intensity of the best comparators are marginal to see reflected part features. The edge breaks can be confused with the part edge. The 50X magnification makes many part images too large for a 30 inch comparator. This required the use of expensive fixtures so the part could be shifted to check portions of the part separately. This is a slow and tedious process.

The Virtual Optical Comparator uses a CCD camera to obtain the image of the part feature. This allows part surfaces to be more easily illuminated and viewed. Parts would be mounted on a precision $\mathrm{X}-\mathrm{Y}$ stage so that the part image can be viewed in one set up. A CAD image of a traditional comparator chart would be overlaid over the part image on the monitor. It could be set and rotated to fit it to the part similar to traditional comparator charts. The part features can then be checked within the CAD image lines the same way traditional charts are used. Two advantages of this system are superior edge detection, and no vinyl charts procurement or inspection. Furthermore, the part size and expensive fixtures are no longer a concern because of the long range of the X-Y table; as the table moves the CAD image will move precisely in synch. Product redesigns require only changes to the CAD image. Additionally, the inspection process will become more ergonomically efficient by allowing the operator to view the part sitting at a desk rather than standing over a 30 inch screen. The procurement cost for the VOC will be less than a traditional comparator with a much smaller footprint with less maintenance and energy requirements. 


\section{Discussion}

\section{Scope and Purpose}

The purpose of this project is to determine the feasibility of using CAD created overlays with a CCD camera, a Virtual Optical Comparator, to measure product limits. The current use of optical comparators is not suitable for use with many current W76 Stronglink parts and development WR1 stronglink parts. The scope of this project is to develop software, design, and build a Virtual Optical Comparator. The system will be evaluated to determine its suitability for use and ability to replace the optical comparators currently in use.

\section{Prior Work}

The first instance of Honeywell FM\&T using a CCD display with a digital overlay was for contact inspection for an MC4713. The contacts needed to be adjusted within $+/-.001$ inch. The contacts for a previous similar product (MC3600) used an optical comparator and an overlay. The contact adjustment and acceptance was very difficult and tedious. The MC4713 uses a flat screen monitor and a computer generated overlay to inspect the contacts. The overlay used was fixed and the part was manipulated to check multiple contacts. The contacts are easily visible and the contacts can be seen as they are manually adjusted.

From this concept, it was conceived that if the digital overlay could be moved with the part it would be possible to inspect parts in a similar manner as an optical comparator but with the advantages of better optical resolution and greater range.

\section{Activity}

After the Virtual Optical Comparator was determined to be a suitable project an order was placed with Industrial Products Group, Inc in Minneapolis. They had already built the video equipment to inspect some electrical contacts using a digital overlay and were well suited to develop this project. The concept was to build a low cost chassis and concentrate on developing the software and hardware to create a moveable digital overlay. The pattern wheel for the WR1 DSSL stronglink was used as a test part. The TSL Pattern Wheel was a good candidate because it contains most of the difficult to measure features we expect to see in the future.

The chassis consists of a 6" X 6" x-y table and a fixed camera mount. The table uses Sony glass scale encoders accurate to +/- .0001“. The camera is a 1 megapixel Hitachi with a 1" x 1 1/8” chip. It uses a Computar TEC-55 telecentric lens. A Samsung Synchmaster 243T LCD monitor is used for a display. It is a 24” unit with 1920 x 1200 lines of resolution. 
The digital overlays are created from the PRO E model drawings of the part. The drawings are modified as required to add contour and positional limits. This file is saved in a DXF format then converted to a TIF image. The two overlays for the Pattern Wheel were created in one hour. For calibration purposes a similar overlay was created to match a calibrated glass grid. A 1 or 2 inch square is drawn around the part image. The square is used by the software to locate and orient the digital image. The appropriate part datum must be located at the center of the square. The resultant TIF images are 12MB in size.

Below is an image of the test system.

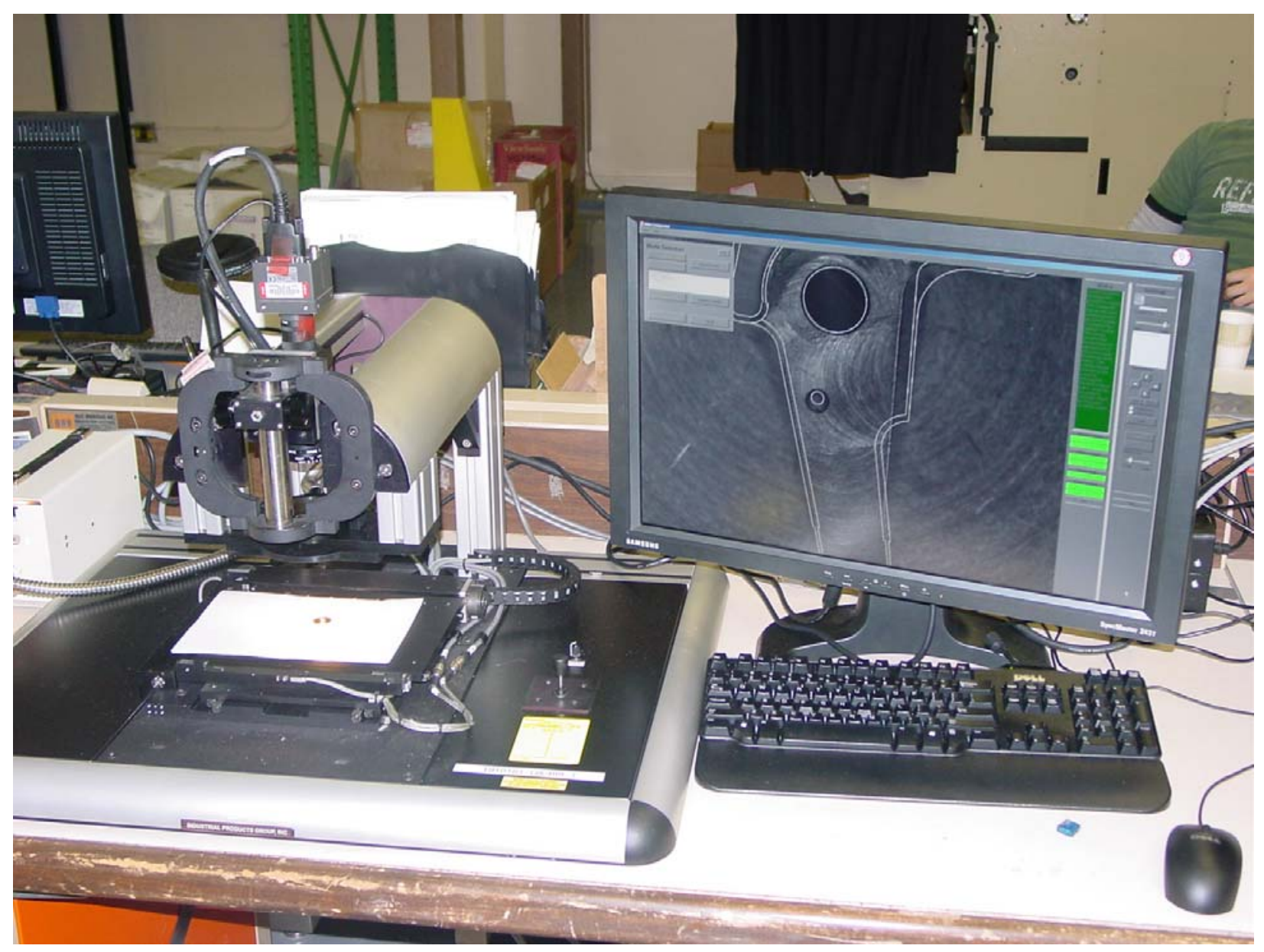

Figure 1. Picture of System 


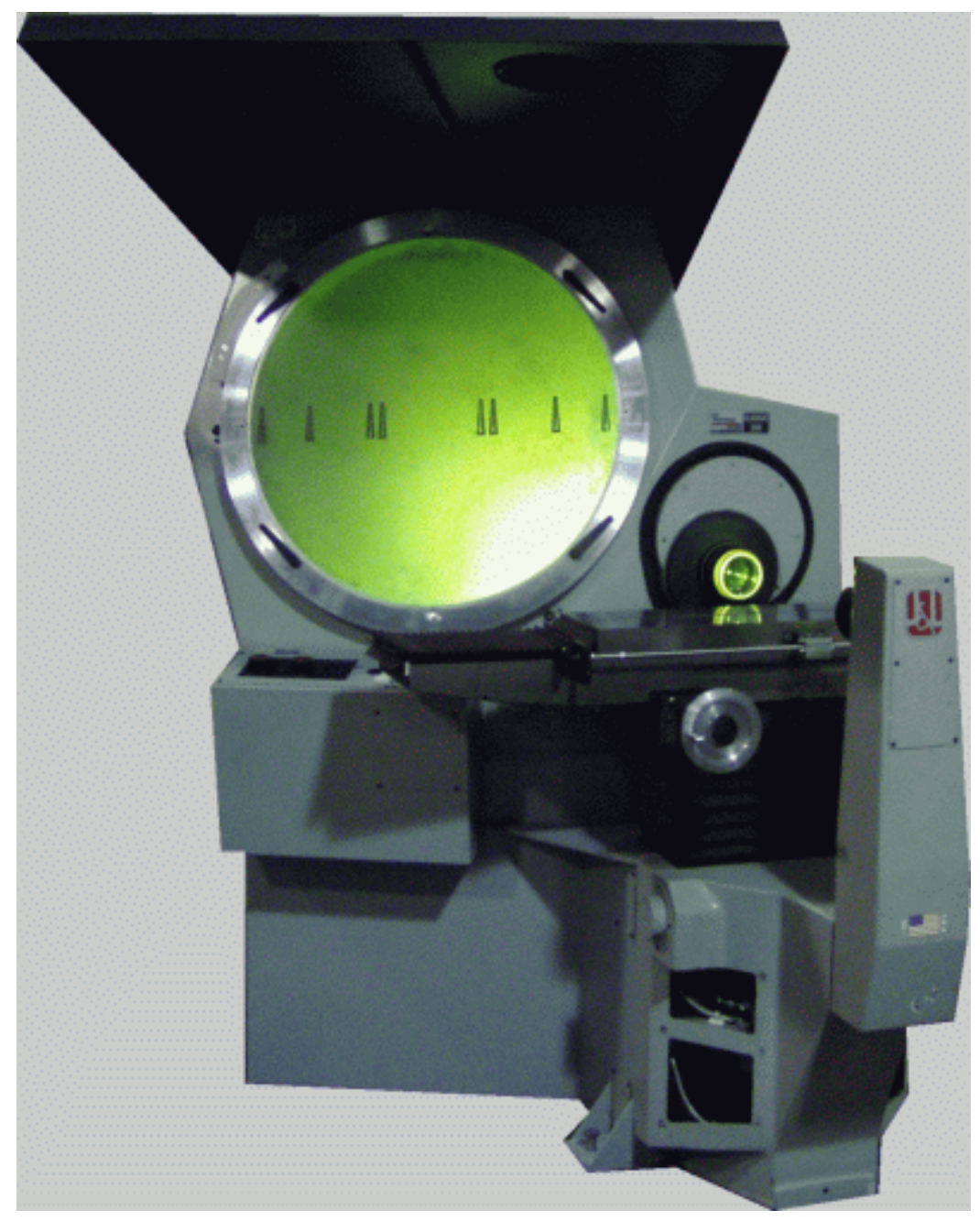

Figure 2. Traditional Optical Comparator 
Below is the sample part that was used to verify the concept. The test is inspecting the .0016 contour on page two.

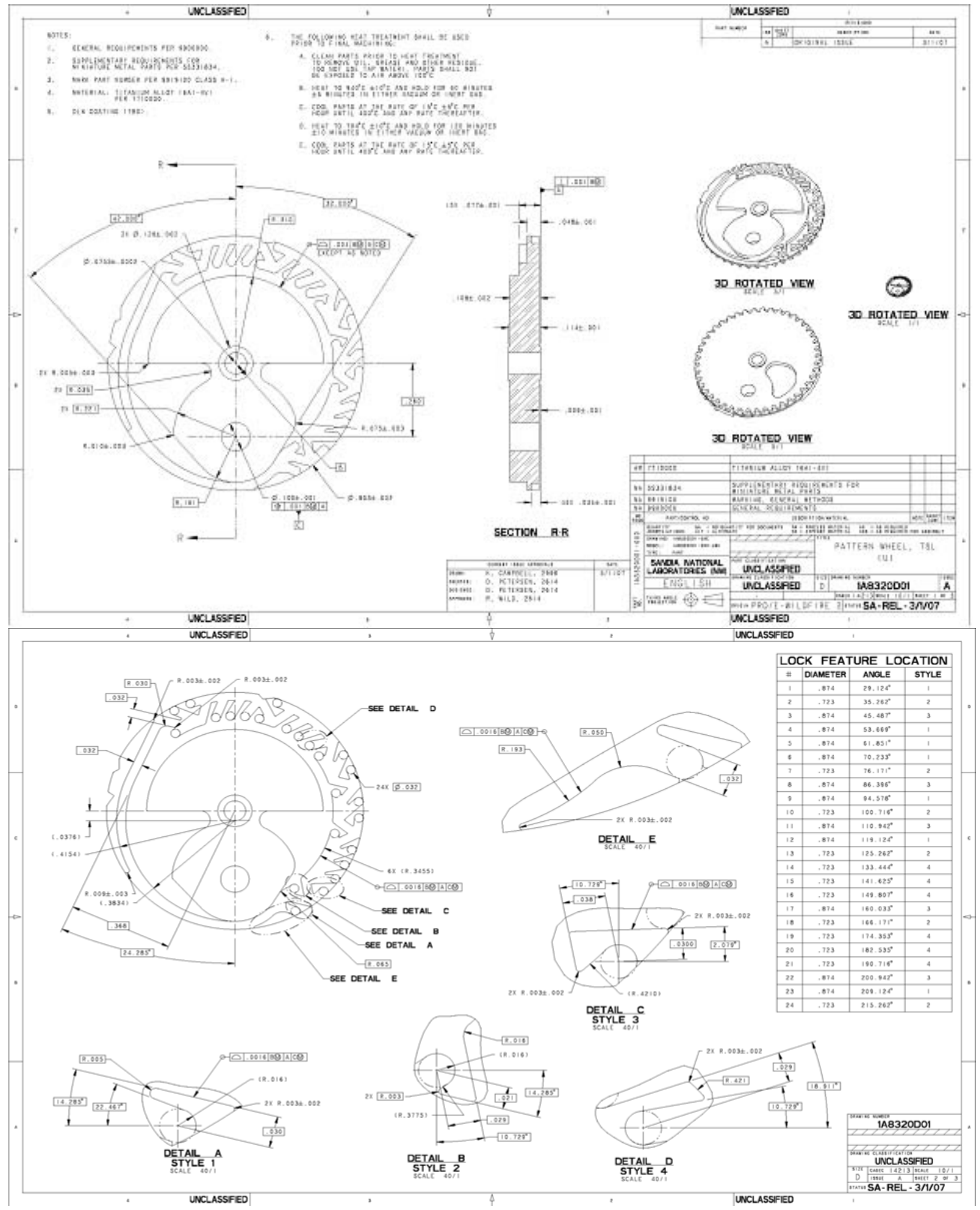

Figure 3. Sample Part Drawing 
This is the digital overlay that was created from the Pro-E model of the part. This file is loaded into the program memory. It takes approximately 30 minutes to create the overlay.

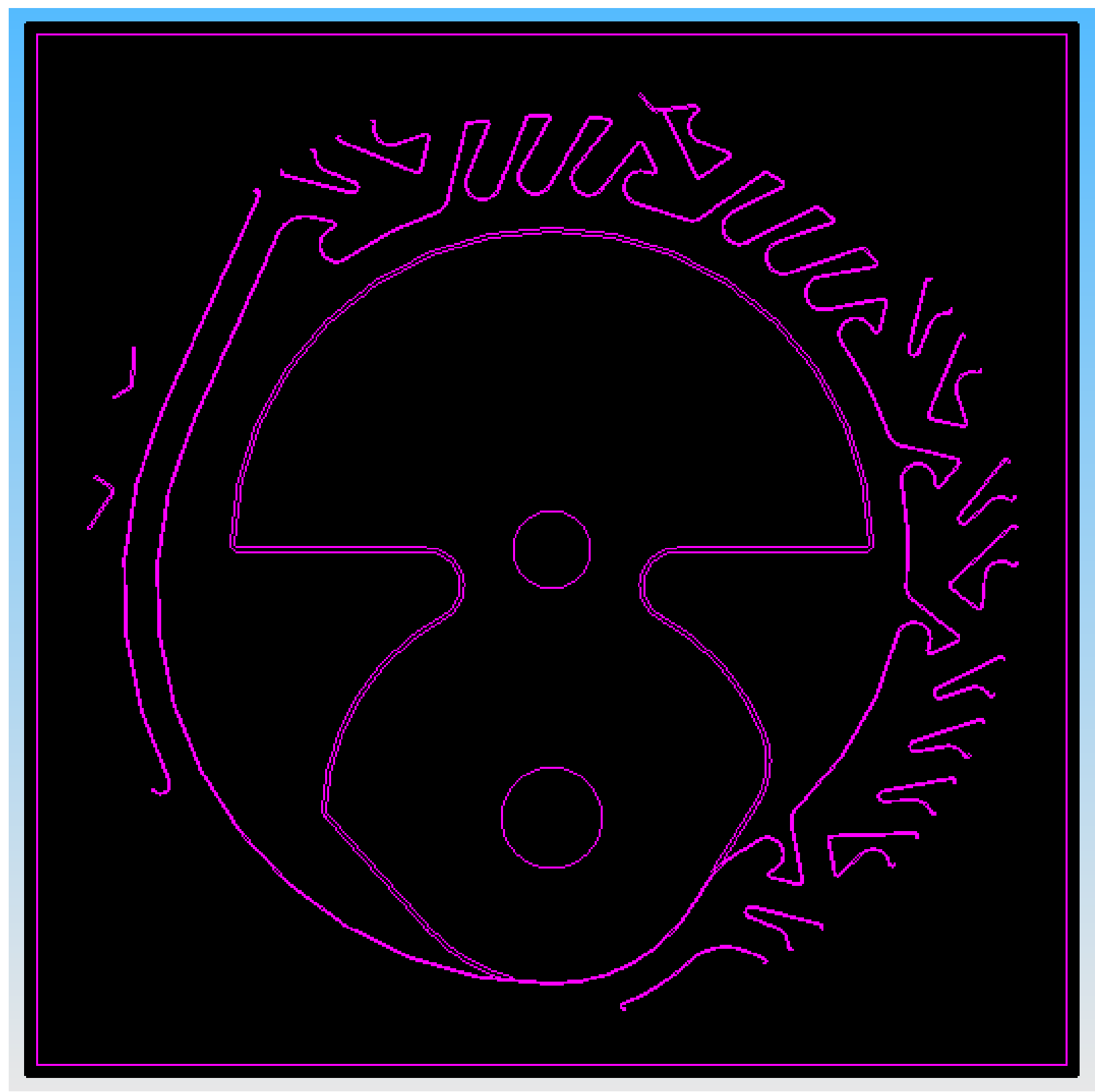

Figure 4. Digital Overlay 
First align the center circle of digital overlay with the part datum B using the X-Y adjustment tools.

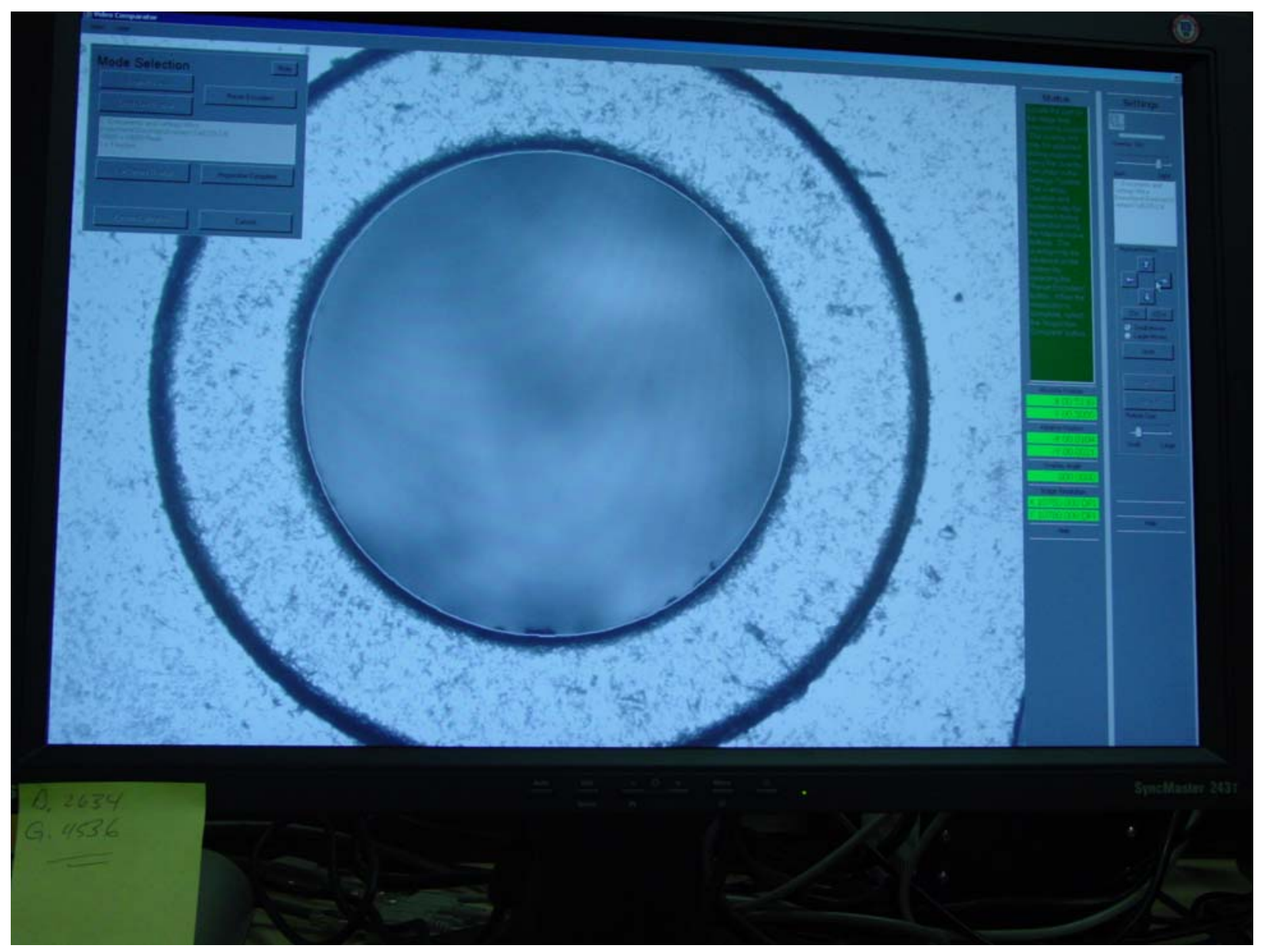

Figure 5. Screen Shot of System Use I 
Then move the image with the joystick to observe part datum C. You can see the digital overlay is not aligned.

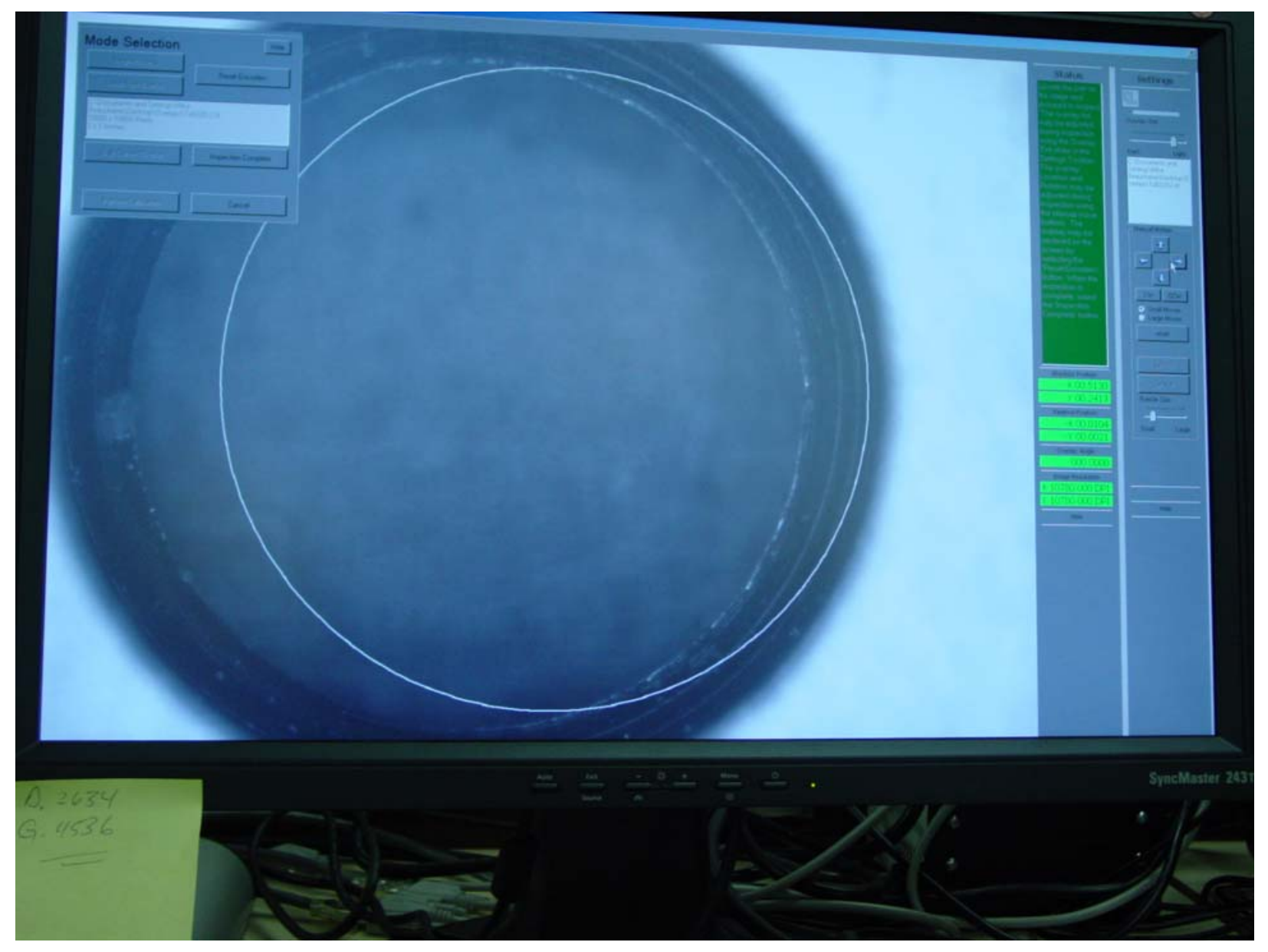

Figure 6. Screen Shot of System Use II 
Using the rotation tool you can rotate the image about the center circle and align it with part datum C. The center circle will remain balanced on part datum B.

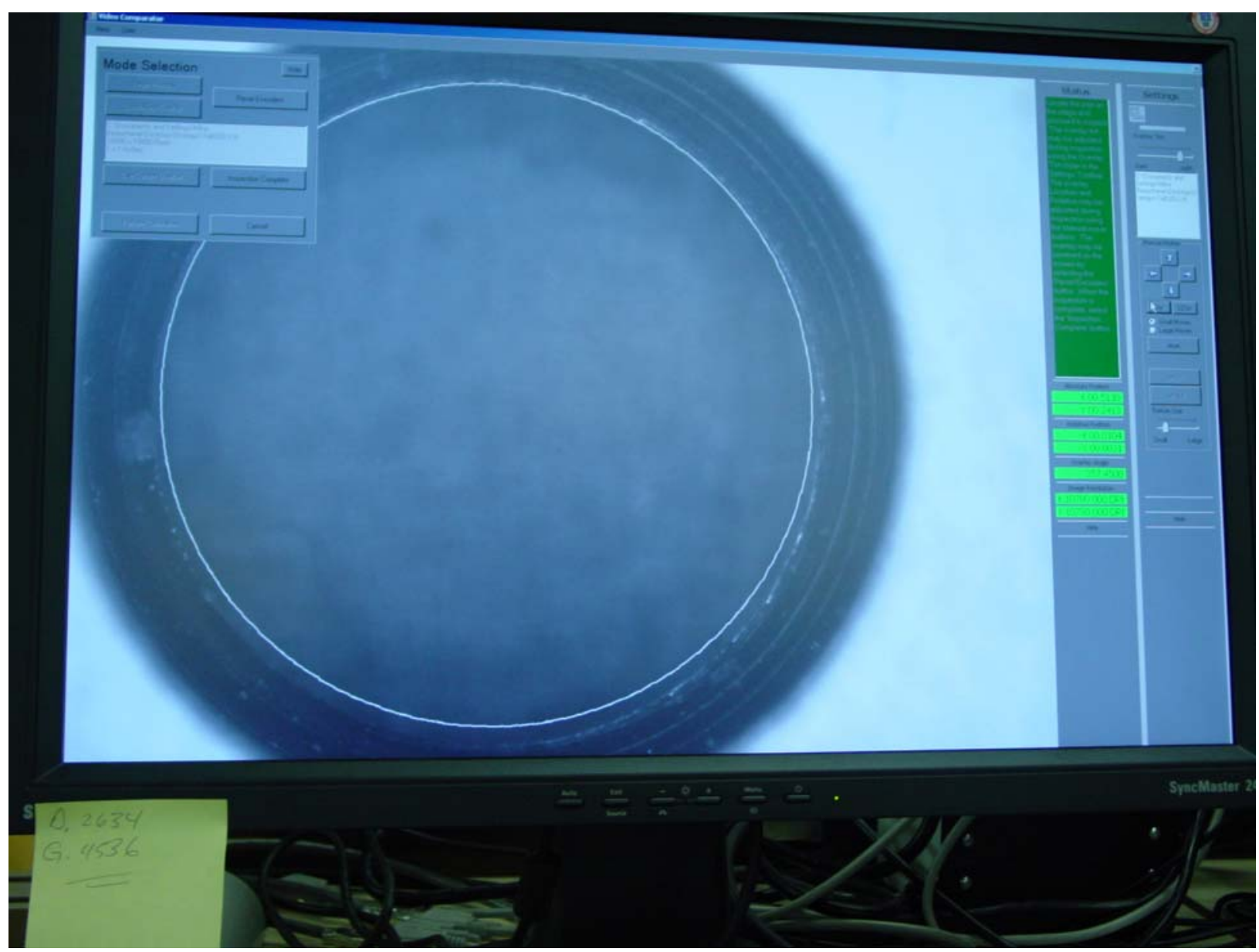

Figure 7. Screen Shot of System Use III 
The joystick is used to position the image along the part contours. The part image should remain inside the two overlay lines. The part we are using did not conform to the drawing.

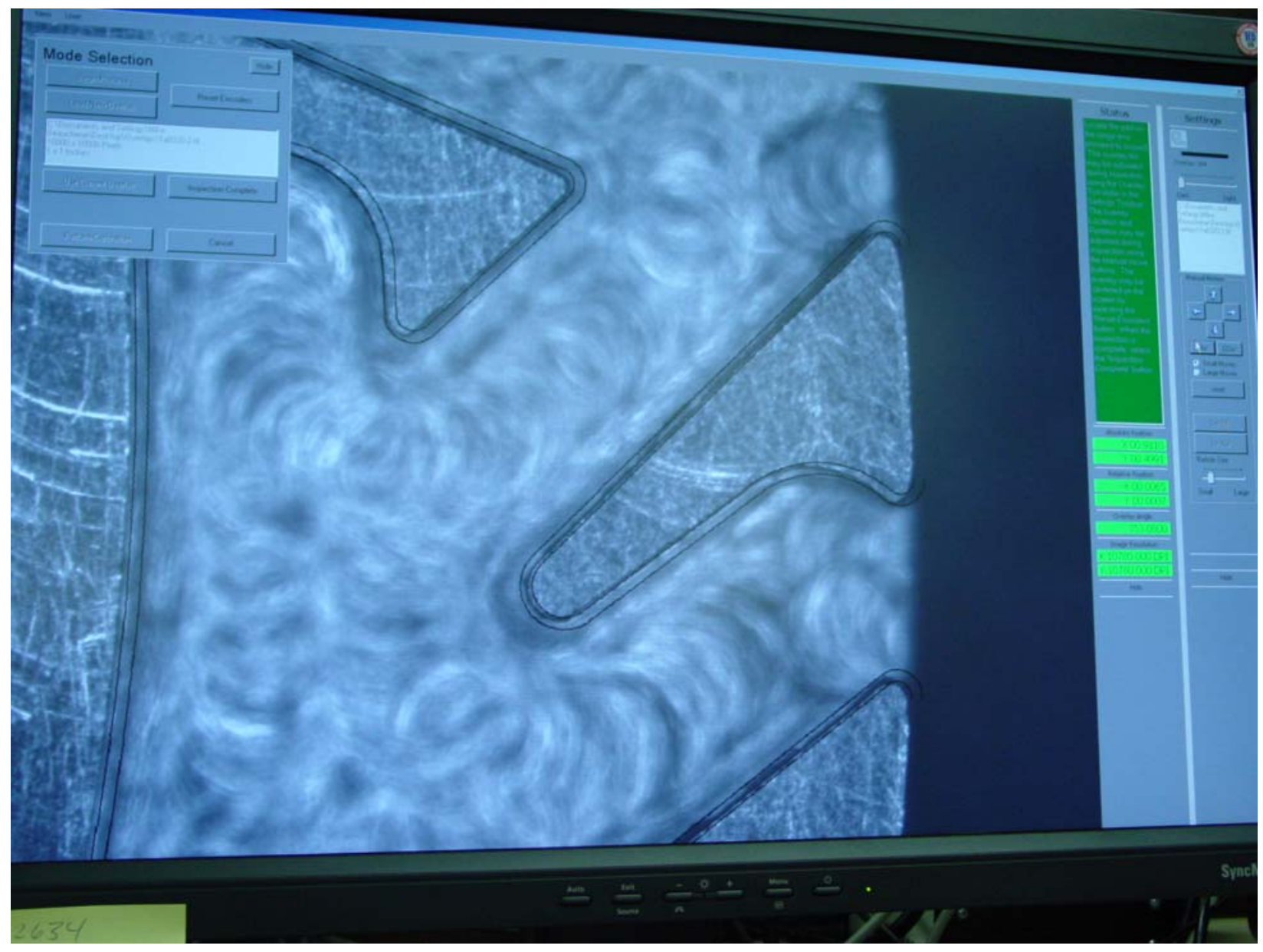

Figure 8. Screen Shot of System Use IV 
Another view of the contour.

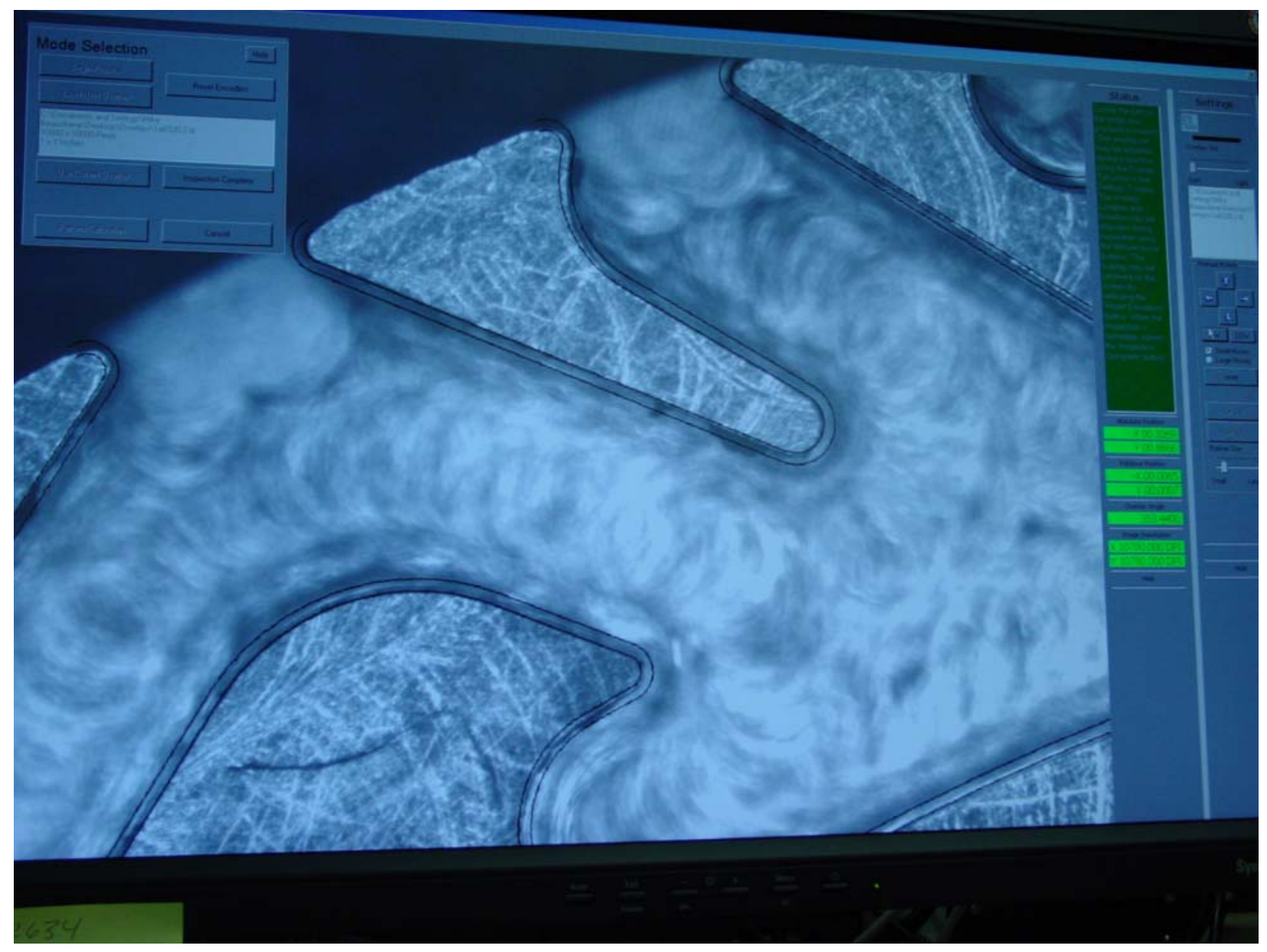

Figure 9. Screen Shot of System Use V

The inspection results seen here correlated with the CMM data that had been taken on the part. 


\section{System Calibration}

The concept of a visual, on-screen "overlay" that represents the calibration standard (or, in the practical sense, a part to be measured) makes the calibration process much more intuitive and thus, simpler to implement.

The current system could be expected to achieve a certification in the range of \pm 0.0002 to \pm 0.00025 inch (XY Planar). The current system was examined only over a range of approximately 1 square inch. Optical Comparators are currently calibrated to +/-.0003 inch at $100 \mathrm{X}$ magnification.

To aid calibration, additional software tools were requested for setting up the measurement origin and alignment (rotation of skew), such that the calibrator could set relative (part coordinate system) zero at a point of his/her choosing. Also requested was a crosshair-type mechanism that would be available on-screen. This mechanism would be used as a sort of datum to make measurements of deviations from nominal geometry (i.e. to quantify the amount that a part feature deviates from the nominal or allowable).

\section{Accomplishments}

This project validated the Virtual Optical Comparator concept. It showed that a digital image can be created through the PRO-E part model. The image can be modified to add acceptance limits for the part. The image can be stored in Matrix for documentation and SQA. The digital image can be overlayed and dimensionally reference a live video image of a part.

The live part image is superior to traditional optical comparators and will allow simpler and more robust inspection of a stronglink product. The footprint of the system allows its use on a desktop, and it can be used by an inspector seated in a chair. Vinyl overlays will not be required to be designed, fabricated, or calibrated. The flow time for overlays can be reduced from several months to several days. The costs for overlay fabrication and calibration will be eliminated.

\section{Future Work}

The activity will involve identifying and procuring an existing commercial chassis for the system, and investigating CCD cameras that will provide greater depth of field and resolution along with newer monitors. Additionally, the software will be modified for easier calibration and basic operation. This system will be formally calibrated and used on existing products. The goal is to be able to produce a system costing $\$ 40 \mathrm{~K}$ or less that can replace traditional optical comparators. 


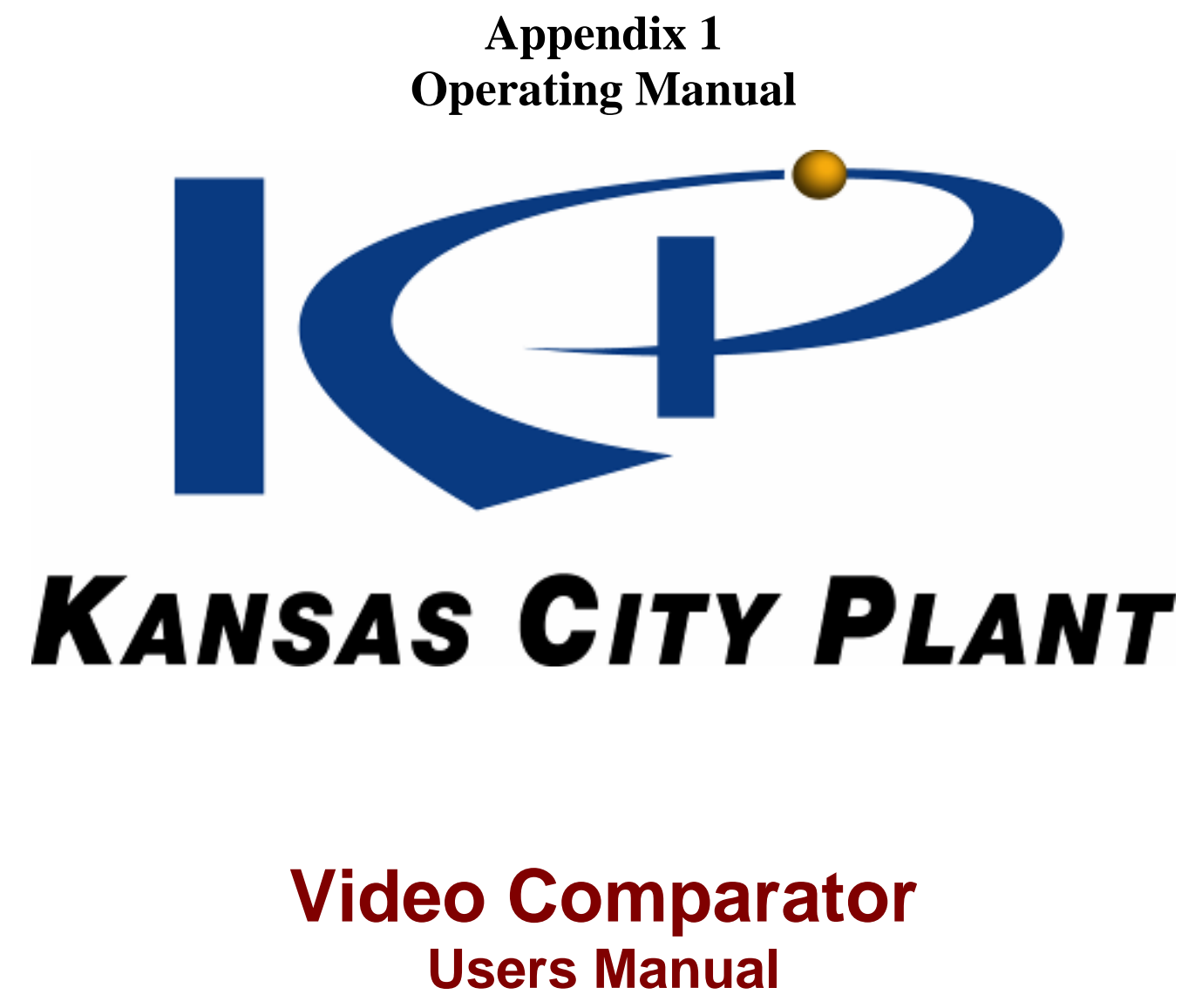




\section{Table of Contents}

1. Introduction 3

a. System Overview 3

b. Specifications 3

2. System Setup

a. Camera Calibration 4

b. Stage Calibration 10

c. Windows Settings 11

3. DXF Conversion 11

a. Concepts 11

b. DXF conversion procedure 12

4. Operation 14

a. Powering on the system 14

b. Loading Parts for Inspection 15

c. Loading image For Overlay 15

d. Aligning Part with Overlay 17

e. Inspection 18

f. Measurements 18

5. Maintenance 18

a. Cleaning 18

b. Lubrication 19 


\section{Introduction}

\section{1-A System Overview}

The Video Comparator is designed to import native CAD drawings, in DXF format, to create an overlay which then can be used to compare with a live image of the object to be inspected. The primary hardware components consist of:

- A high-resolution monochrome video camera

- An X-Y motorized stage, controlled by a joystick

- A polarizing ringlight and analyzer

- Large screen color monitor

- Computer with image capture and processing capabilities.

Utilizing custom software, the Video Comparator will display an overlay on top of a live image with a combined resolution up to $.0001 "$. The joystick can then be used to traverse the stage and compare features anywhere within the limits of the 6" $\times 6$ " travel. Once the overlay is positioned correctly over the live image, the overlay will "pan" with the stage travel. The polarizing fiber optic ringlight and analyzer provides the sharpest, most glare-free image possible on a wide range of materials and finishes.

\section{1-B Specifications}

\section{Camera:}

Hitachi KP-F200CL-S1

\begin{tabular}{|l|l|}
\hline Effective Picture Element: & $1 / 1.8 "$ CCD $1628(\mathrm{H}) \times 1236(\mathrm{~V})$ \\
\hline Frame rate & 30 frames per second \\
\hline Video output & Digital or analog for image checking \\
\hline Sync system: & Internal \\
\hline Aspect ration & $4: 3$ \\
\hline Digital output & Camera-Link \\
\hline Lens mount & C-mount \\
\hline Electronic Shutter: & $1 / 30$ to $1 / 50,000 \mathrm{sec}$ \\
\hline Power Supply: & 12 V DC \\
\hline
\end{tabular}

\section{Lens:}

Computer TEC-55 telecentric lens, 2/3" format, $55.0 \mathrm{~mm}$ focal length, F2.8, manual iris and manual focus. C-Mount

\section{Monitor:}

The Samsung SyncMaster 243T is a pivoting 24.0" analog/digital LCD display that offers a 500:1 contrast ratio, $1920 \times 1200$ resolution, pixel pitch of $0.270 \mathrm{~mm} \mathrm{H} / \mathrm{V}$, Xtrawide viewing angle, scanning frequency of $30-81 \mathrm{kHz}$ horizontal and 56-75 vertical, and dual inputs to accommodate either an analog or digital signal.

Panel Size: 24" 
Brightness (Typical): $300 \mathrm{~cd} / \mathrm{m} 2$

Contrast Ratio: 500:1

Viewing Angle $(H / V)$ : 170/170 (degrees)

Interface: Analog/Digital

Horiz. Frequency: $30-81(\mathrm{kHz})$

Max/Native Resolution: 1920×1200

Pivot Technology: Yes

Special Features: Narrow Bezel Design, Pivot Technology, Ergonomics, Height Adjustment and

Swivel, Dual CPU Input

\section{Linear Encoders:}

Manufacturer: Sony

Model: SR118 Magnetic miniature scale for small-size linear stages

Travel: $15 \mathrm{~mm}$ up to $260 \mathrm{~mm}$

Accuracy: $+/-2 \mu \mathrm{m},+/-2.5 \mu \mathrm{m},+/-3 \mu \mathrm{m}$

Resolution: $0.5 \mu \mathrm{m}$

Reference points: Optional external

Measuring system: Magnetic

Max response speed: $60 \mathrm{~m} / \mathrm{min}$

Protection design grade: To IP44

Air purge: No

Output cable (connector): Hirose M2-7P

Cable length: $300 \mathrm{~mm}$ braided cable, cables 3,5,10,15m.

Output type: Requires Sony interface for A/B phase signal

\section{Computer and Login:}

Manufacturer: Dell

Model: $\quad$ Precision 690

Login: $\quad$ Mike Beauchene, no password

\section{System Setup}

\section{2-A Camera Calibration}

Camera calibration consists of two steps, first calibrating optical magnification, second calibrating of camera bracket alignment. In order to calibrate the optical magnification, an NIST certified reference standard is recommended. The ideal reference standard should consist of a rectangular target slightly smaller than the camera field of view (FOV). Since the FOV is fixed at $\approx .070$ " (horizontal) x .053" (vertical), a reference standard with marks about .063 apart horizontally and .048 apart vertically (or about $90 \%$ of the field of view) will give maximum accuracy. For the purposes of this manual, a reference standard with a dot pattern was used to show the procedure.

Camera Calibration Procedure: 
1. Launch the Video Comparator software by either selecting the Start $\rightarrow$ All Programs $\rightarrow$ IPG $\rightarrow$ VideoComparatorProject, or by double-clicking the icon on the desktop.

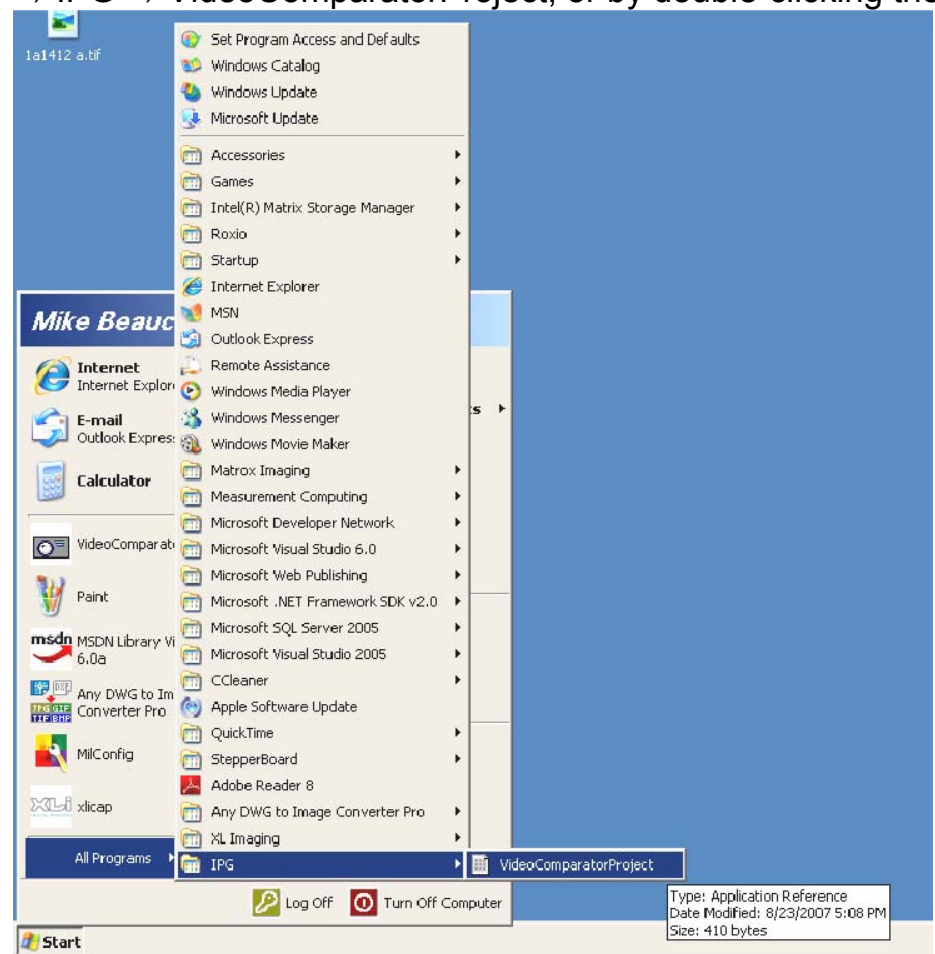

Figure 1

2. Once the program opens, select "Begin Process" in the Mode Selection dialog box.

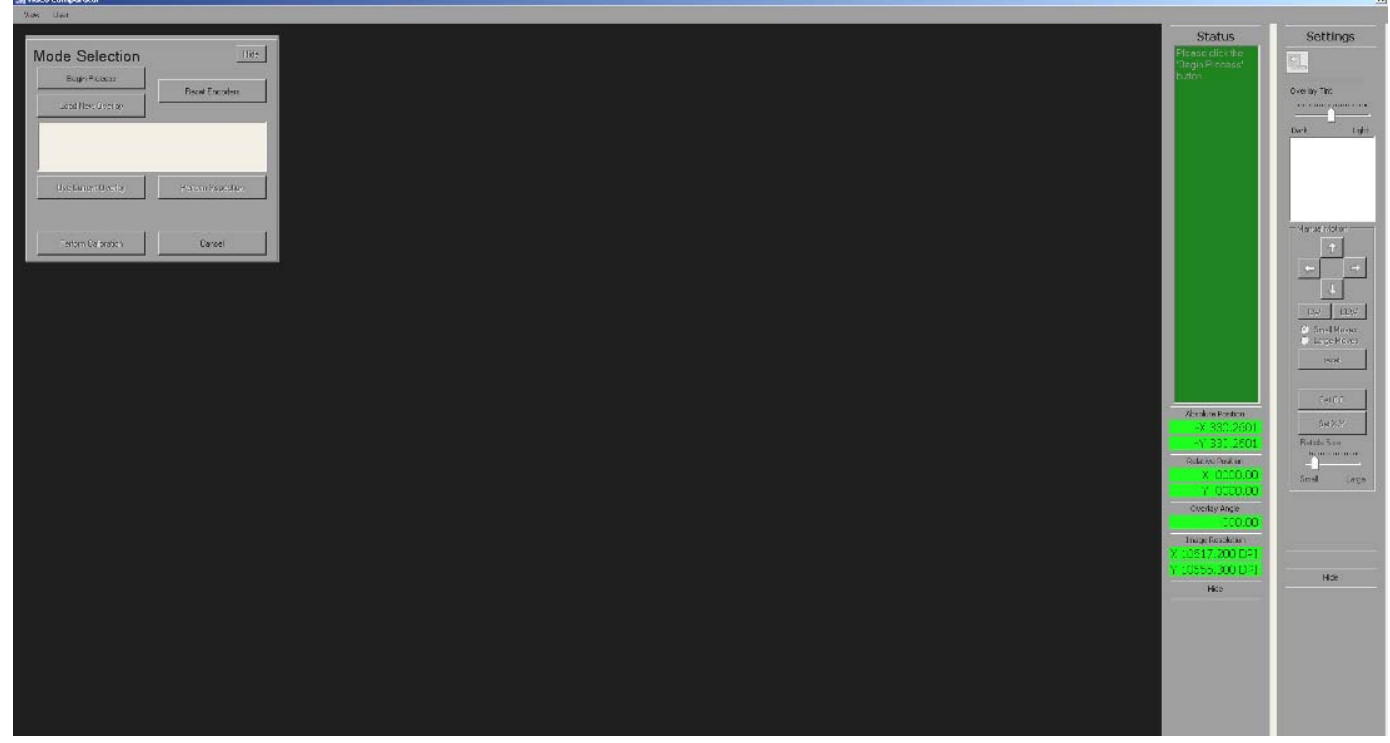

Figure 2

3. Place the reference standard on the stage surface. Adjust the illumination and focus height of the camera to give the sharpest image. Align the standard with the edges of the field of view, and 


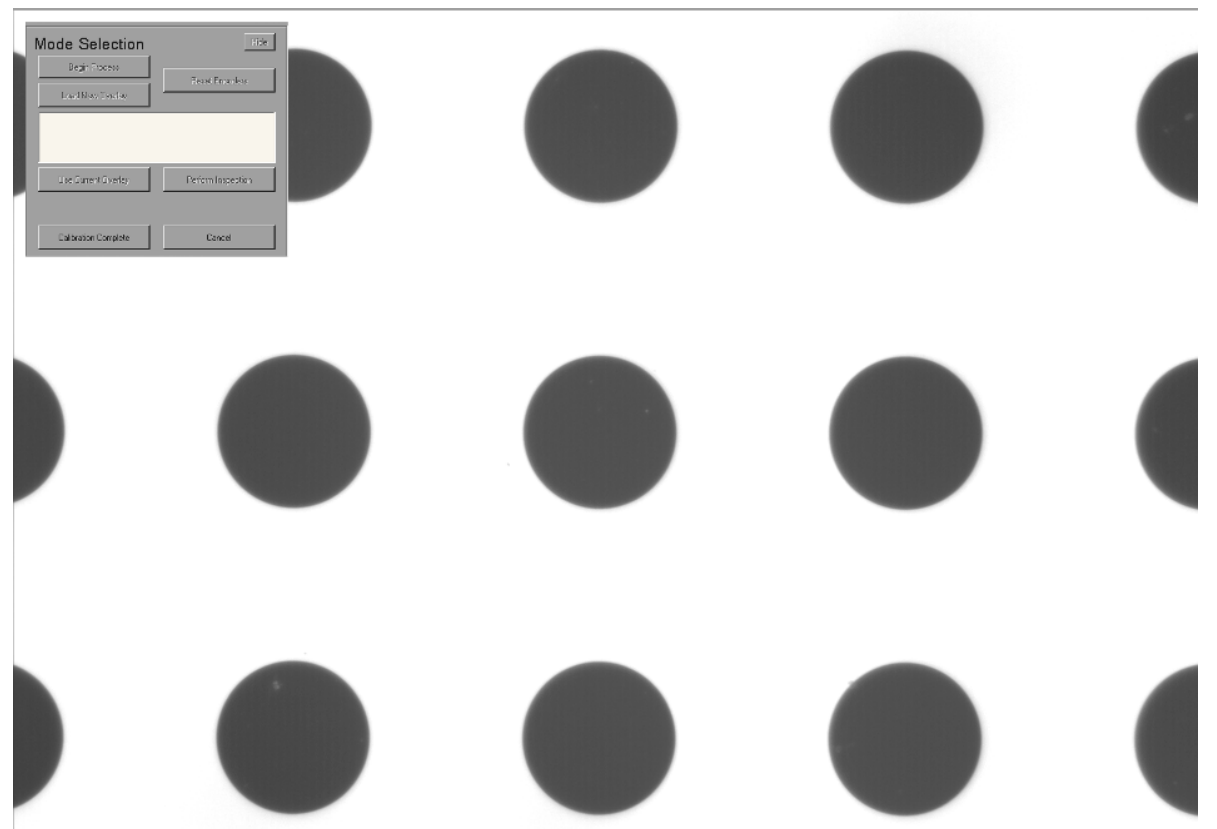

Figure 3

4. From the Menu bar, select "User" $\rightarrow$ Login, Figure 4.

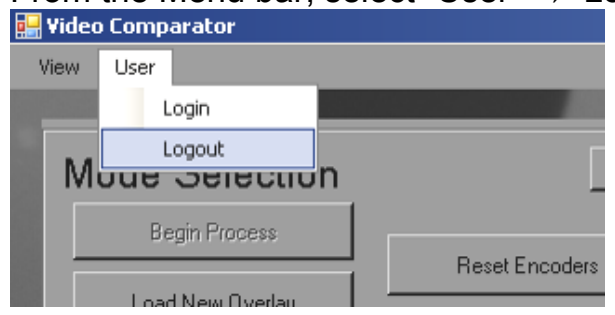

Figure 4

5. Enter the default login name: "Joe" and the default Password: "Joe". In the Setting Dialog box that opens, Figure 5, you may change the user name and password.

On the CalibrationX and the CalibrationY lines, enter the respective distances between the reference marks on the standard you are using to calibrate the optics, Figure 5.

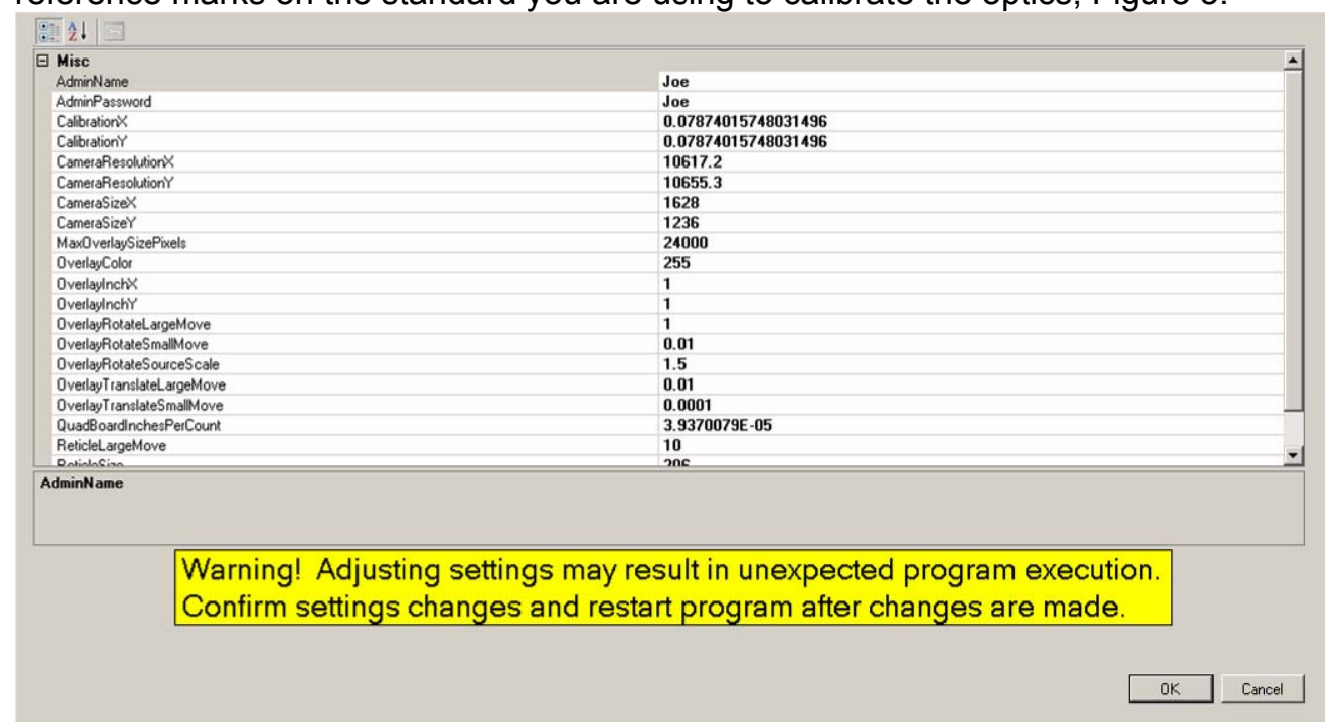

Figure 5 
Click OK to close the dialog box.

6. Press the "Perform Calibration" button in the Mode Selection dialog box, Figure 6

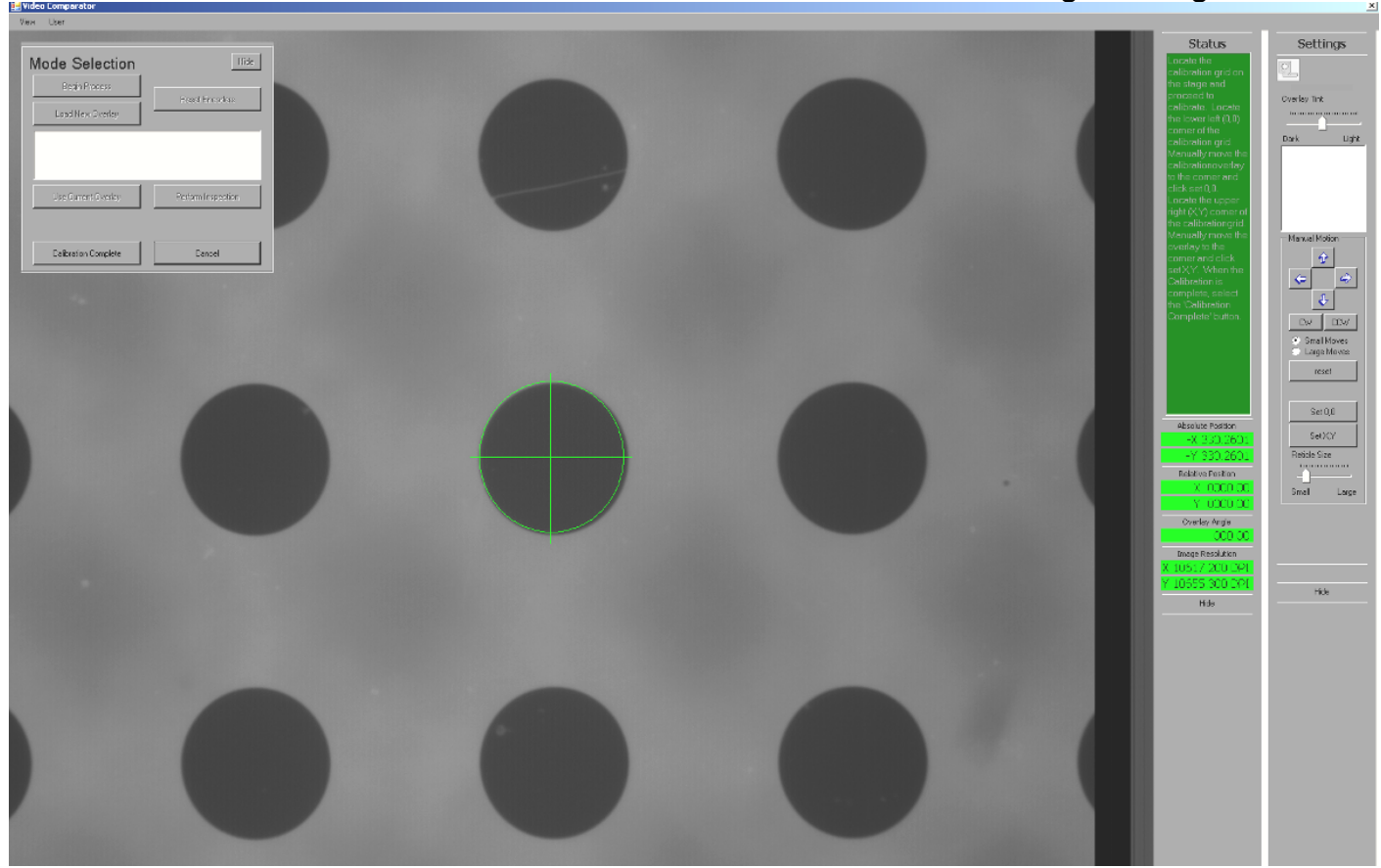

Figure 6

A flashing reticle will appear in the center of the FOV. Use the arrow buttons in the Setting pane on the far right-hand side of the screen to move the reticle to the lower left-hand target on the reference standard. The amount the reticle moves for each click of the arrow button can be adjusted from "Small Moves" to "Large Moves" in the Manual Motion area of the Settings Pane: Figure 7

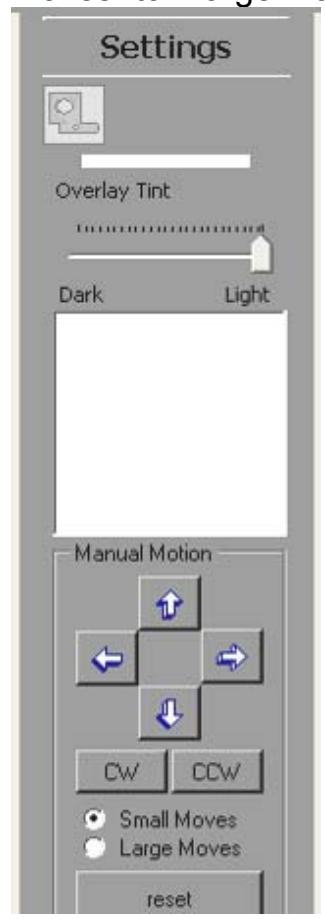

Figure 7

Adjust the size of the reticle circle by moving the adjusting slider, Figure 8. 


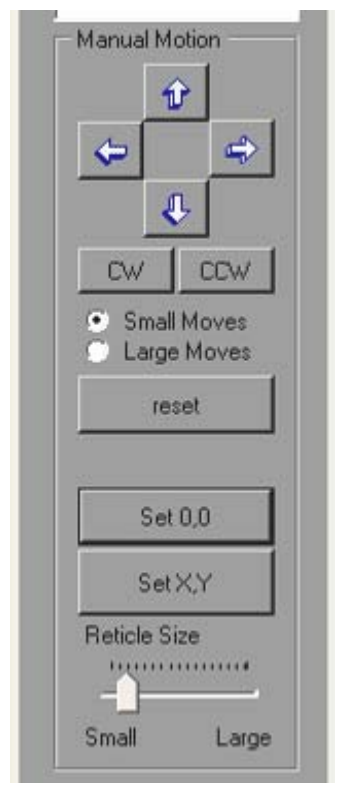

Figure 8

7. Once the reticle is positioned as close as possible over the lower left-hand target, click the "Set 00 " button.

8. Move the reticle using the arrow buttons to the upper right-hand target. Once the reticle is positioned as close as possible over the right hand target, click the "Set XY" button.

9. The camera and optics are now calibrated.

10. Now after calibrating the magnification, the camera adjustment bracket must be calibrated. (The bracket has been calibrated before shipping) There are 3 axis about which the camera must be calibrated:
a. Roll
b. Pitch
c. And Yaw.

The Roll adjustment will rotate the camera about the axis of the lens. This must be aligned with the X-Axis of the stage motion. Place a straight edge on the stage surface aligned with the XAxis. Traverse the stage in the $X$ direction and watch the image from the camera to check for edge creep. If the edge creeps as the stage travels in the $X$ direction, adjust the alignment of the straight edge until it remains stationary in $Y$ as the stage traverses in $X$. Use the straight edge to check the alignment of the camera by carefully moving the stage in the $Y$-axis until the straight edge is aligned with the edge of the Field of View. If the camera Field of View is not aligned with the straight edge, loosen the screws in Figure 9, and adjust the Roll until they are aligned. 
Tighten the screws.

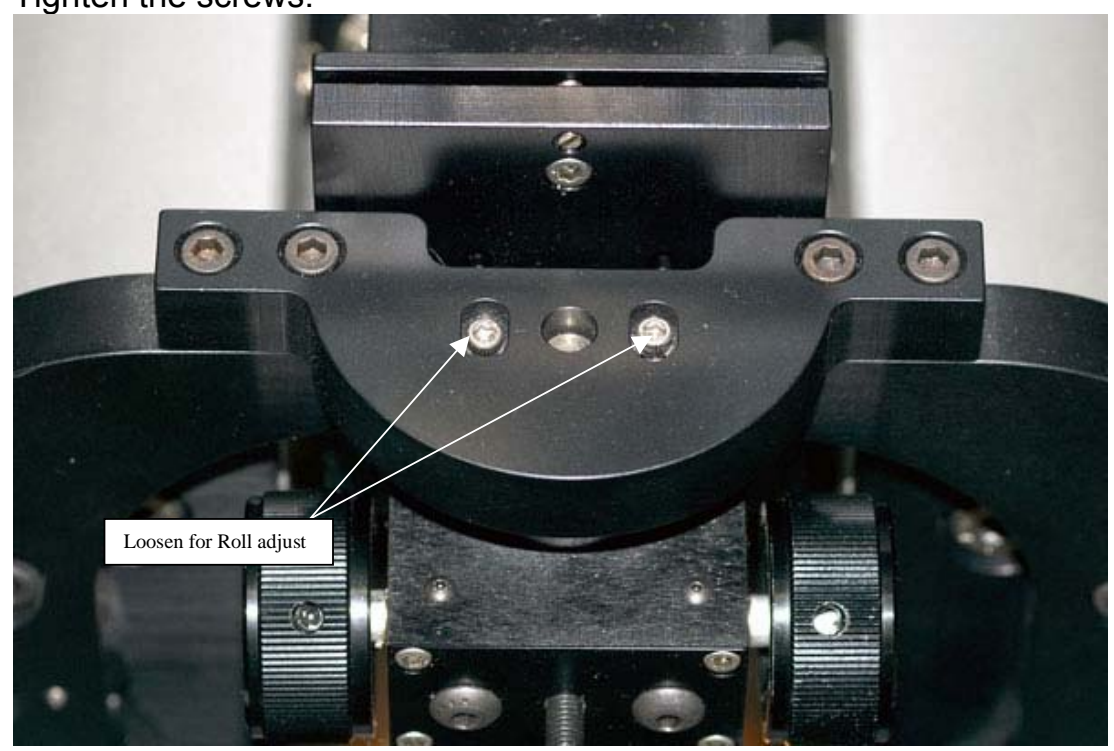

Figure 9

The Pitch and Yaw adjustment will be done by using a flat reference with marks that reach to the edges of the Field of View. Place the reference on the stage surface. Adjust the focus height of the camera for the sharpest image. Carefully inspect the left and right sides of the image for sharpness. If the left and right edges of the image are not both in focus, the Yaw needs to be adjusted. Loosen the screws for the Yaw adjustment, Figure 10, and adjust the Yaw until the image is equally focused on the left and right side of the image.

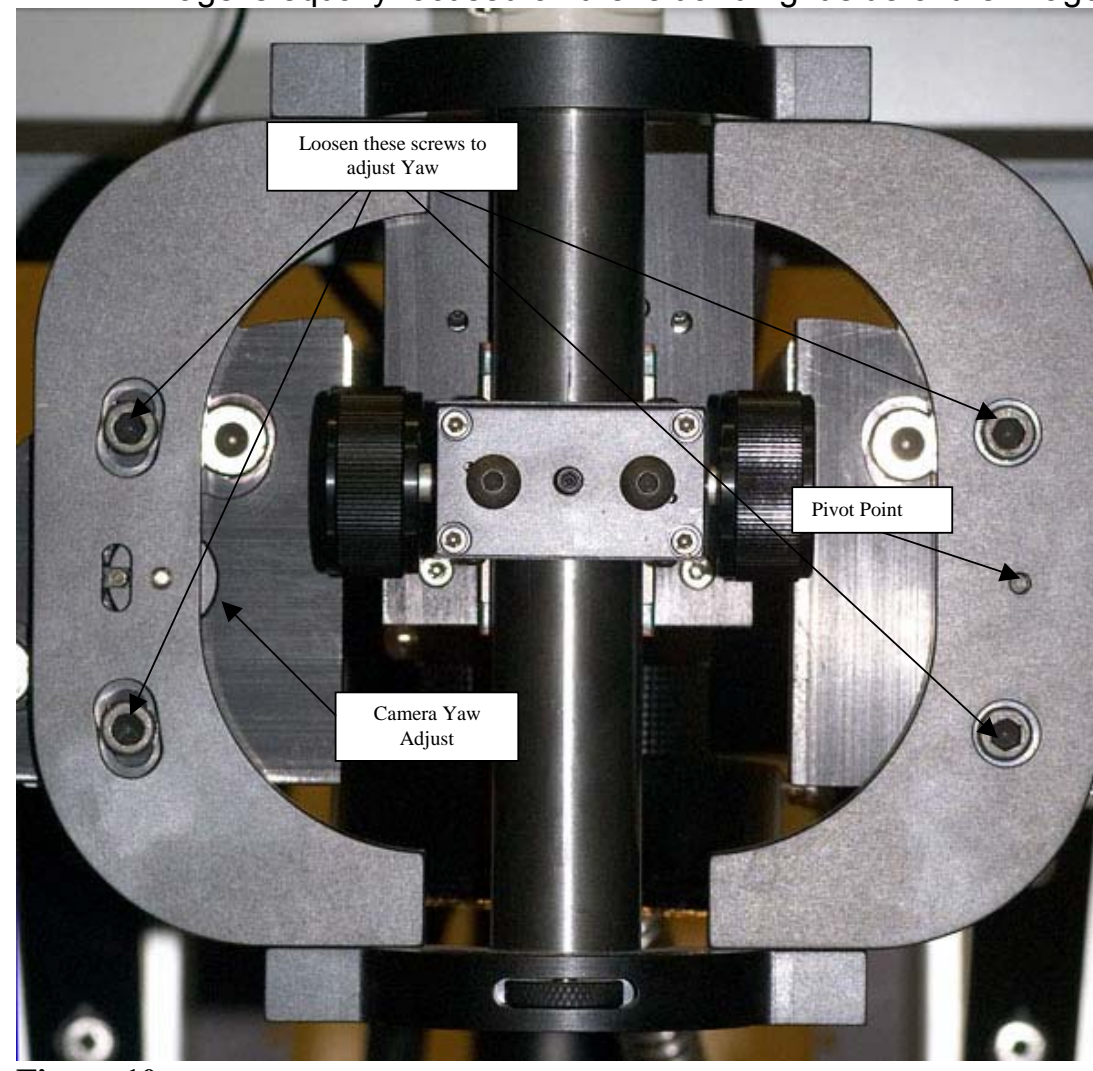

Figure 10 
Repeat the same procedure using the top and bottom of the image for the Pitch adjustment. If the top and bottom of the image are not equally in focus, loosen the 3 Pitch Locking screws in Figure 11. Carefully adjust the Pitch Adjustment screw to make changes in the Pitch of the camera. When the image is equally in focus from top to bottom, tighten the Pitch Locking screws.

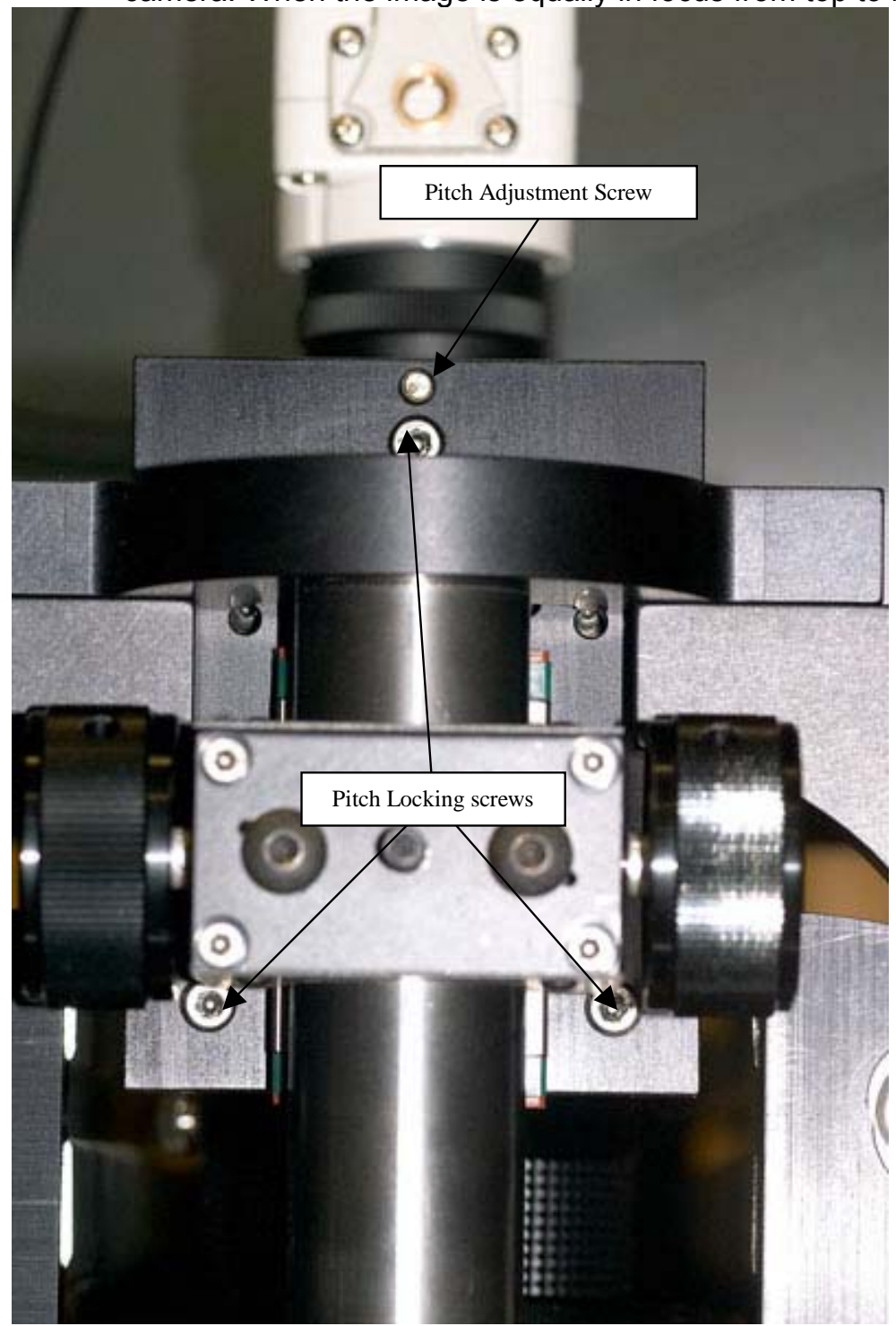

Figure 11

The Camera and Optics are now calibrated.

\section{2-B Stage Calibration}

Stage Calibration requires the use of a glass standard or precision ground square or rectangular gage block. Then loading a DXF overlay of the standard (as covered in section 4-C) then using the overlay you will be able to study the status of the stage alignment. If adjustment is needed the stage may be adjusted.

The $X$ and $Y$ axes of the stage pivot about each other from a center pin. Located out from the center you will find four $1 / 4-20$ socket head cap screws that lock the two axes in alignment. To adjust, these screws must be loosened, Figure 12. 


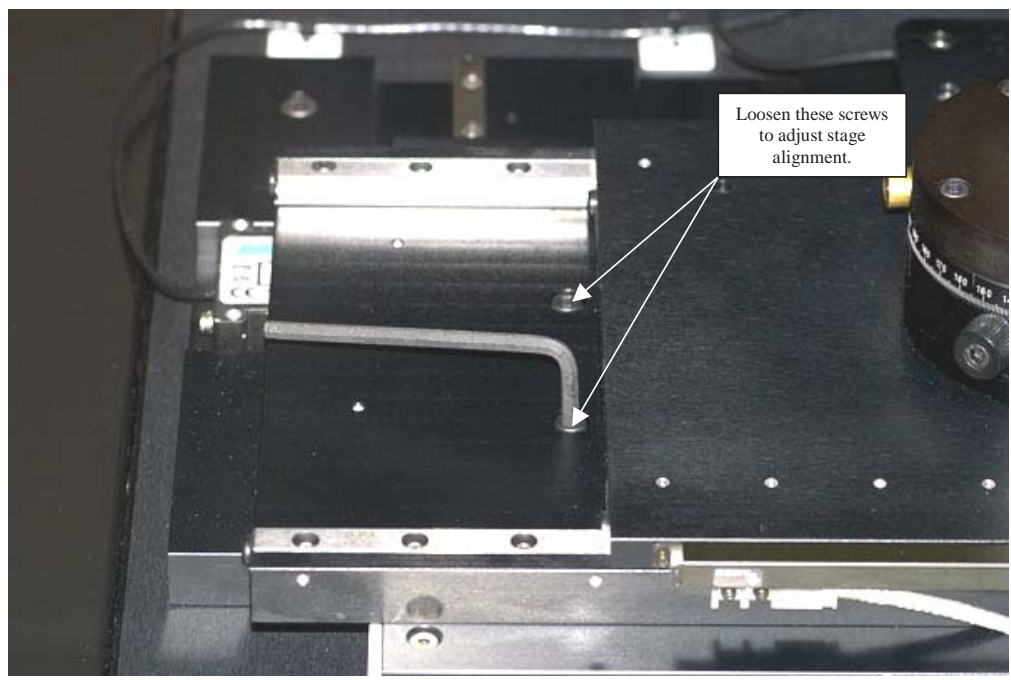

Figure 12

\section{2-C Windows ${ }^{\circledR}$ Settings}

The Windows ${ }$ settings are optimized for performance. These settings must be maintained for the consistent operation of the video comparator software. The video comparator software is very resource hungry especially when it comes to system memory. All other programs should be terminated before starting the video comparator software program. No other programs should be run at the same time as the Video comparator to avoid memory conflicts.

\section{DXF Conversion}

\section{3-A Concept}

In this section we will cover the basic concepts for DXF conversion for the Video Comparator. The requirements for all DXF files will be:

1. All DXFs will be created with a scale of $1: 1$

2. The DXF file will need to have a 1 pixel boarder.

3. The part to be inspected may have a reference origin which will be centered in $X$ and $Y$ and not exceed the boarders.

4. Line color is black, background is white 


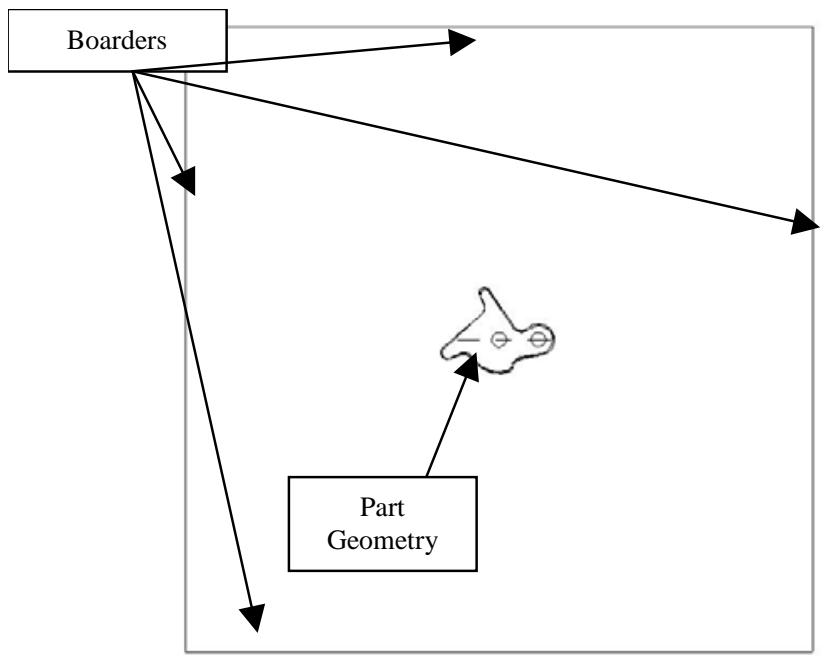

Figure 13

\section{3-B DXF conversion procedure}

Procedure for converting DXF files.

1. Launch the program "Any DWG to Image Converter Pro" from the Start menu or by doubleclicking the icon on the desktop. The interface looks like Figure 14.

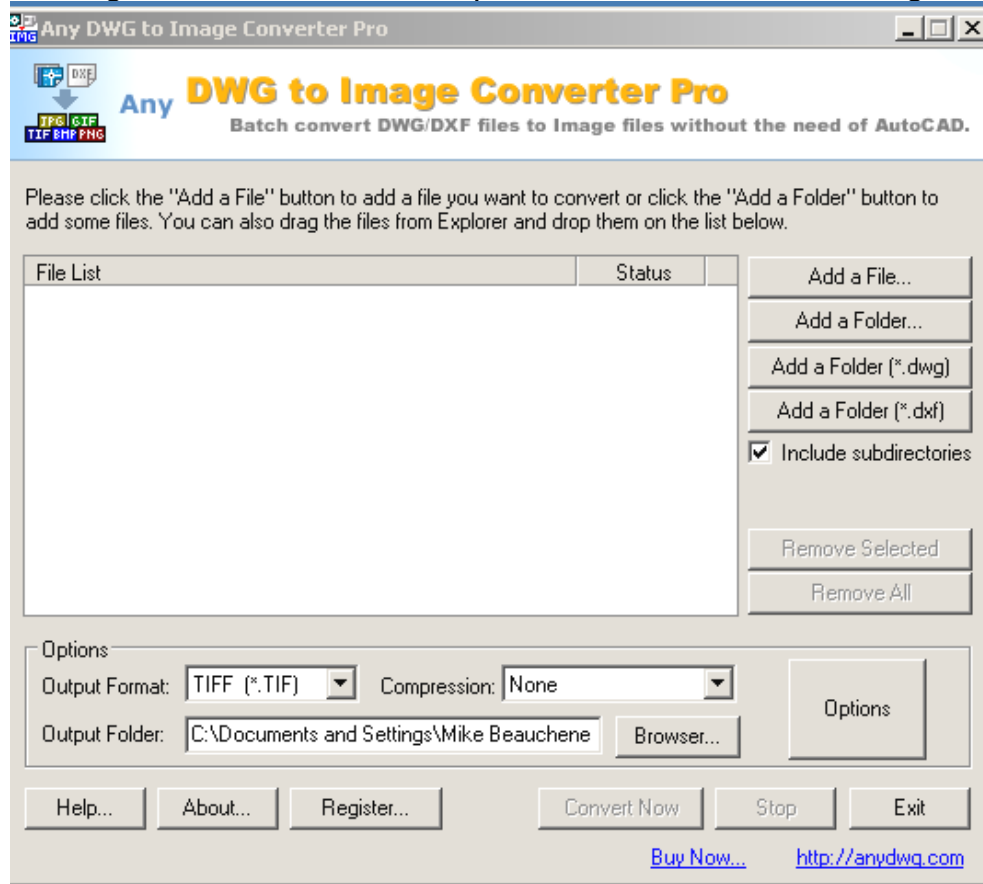

Jnregistered Version - You have 17 times left in the trial.

\section{Figure 14}

Insure that the Output Format in "Options" area is set to TIFF, the Compression is "None" and the Output Folder is where you can find it again (the default is C:IProgram Files, Industrial Products Groupl. 


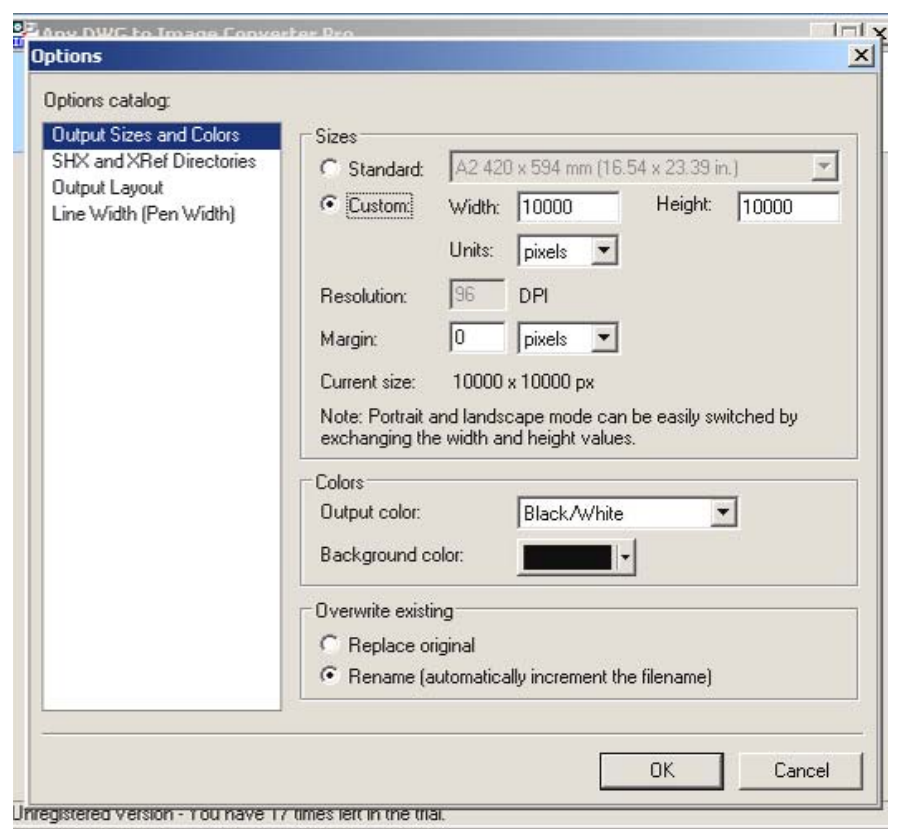

Figure 15

2. Click on the "Options" button. The Options dialog box opens, Figure 15. Under "Output Sizes and Colors" select "Custom" for Sizes. Enter the size of the final image in pixels. Remember to make the pixels the same proportion as the original DXF file size, I.E. if the DXF was a square, enter the same number of pixels for both Width and Height. In general, the higher the number of pixels, the greater the resolution of the resulting image. However, the image must be greater than $1,600 \times 1,600$ pixels and less than $24,000 \times 24,000$ pixels.

3. The "Margin" must be " 0 " pixels.

4. The "Output color" must be "Black/White"

5. The "Background color" must be Black.

6. Select "Rename (automatically increment the filename)

7. On the left-hand frame under "Options Catalog:" choose "Output Layout"

8. Under "Output layout", select "Model space Figure 16

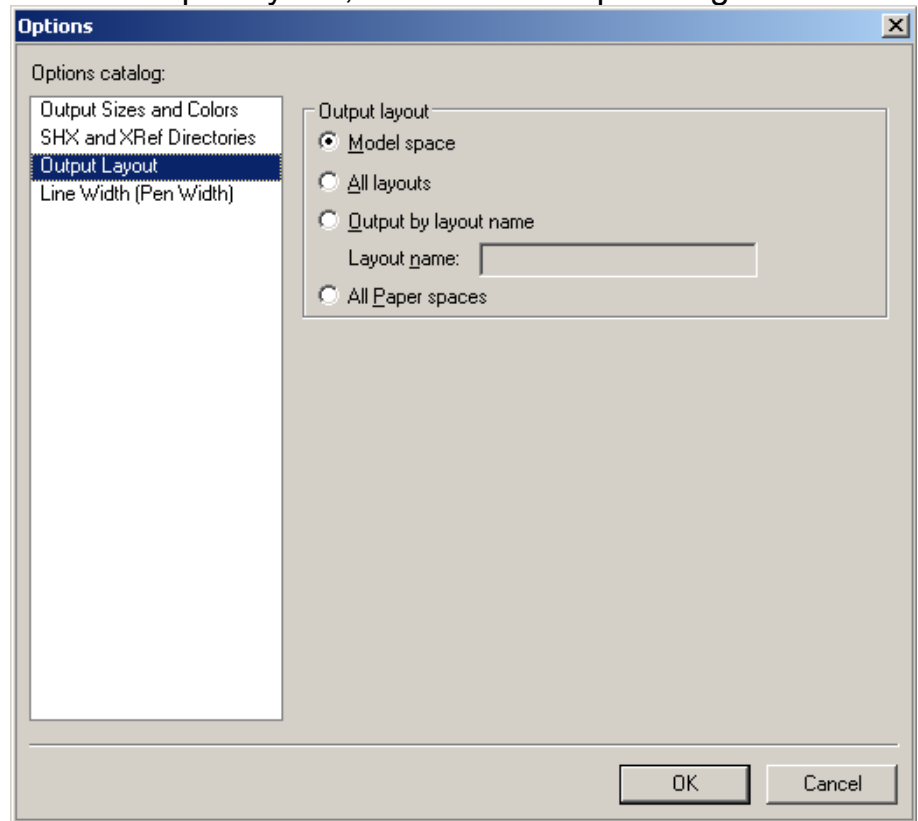

Figure 16 
9. On the left-hand frame under "Options catalog" select "Line Width (Pen Width)", Figure 17.

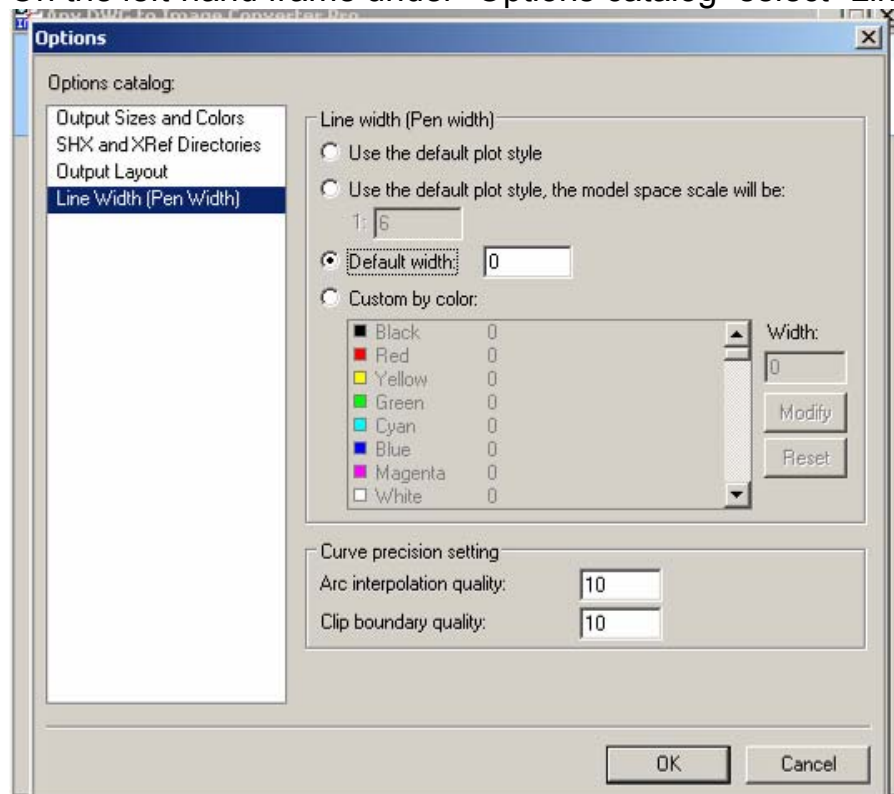

\section{Figure 17}

Under "Line width (Pen width)" select "Default width" and enter "0".

10. Under "Curve precision setting" both "Arc interpolation quality" and Clip boundary quality" may be set as high as " 50 ". The larger the number, the greater the resolution of the converted image.

11. Select "OK" to close the Options dialog box.

12. Select "Add a File" and navigate to the DXF file you wish to convert.

13. Click "Convert Now". A window will open with the new TIFF file in it.

14. You may want to convert a batch of individual DXF files at a time. The program will allow such batch processing.

15. Once you have successfully completed converting a DXF file to a TIFF file, click "Exit" to close the program.

\section{Operation}

\section{4-A Powering on the system}

Turn the power on the system by turning on the power supply for the stage and camera. Turn on the fiber optic light source. Turn on the computer. At the login screen, click the single icon. Once you have logged in, launch the Video Comparator program, Figure 18. 


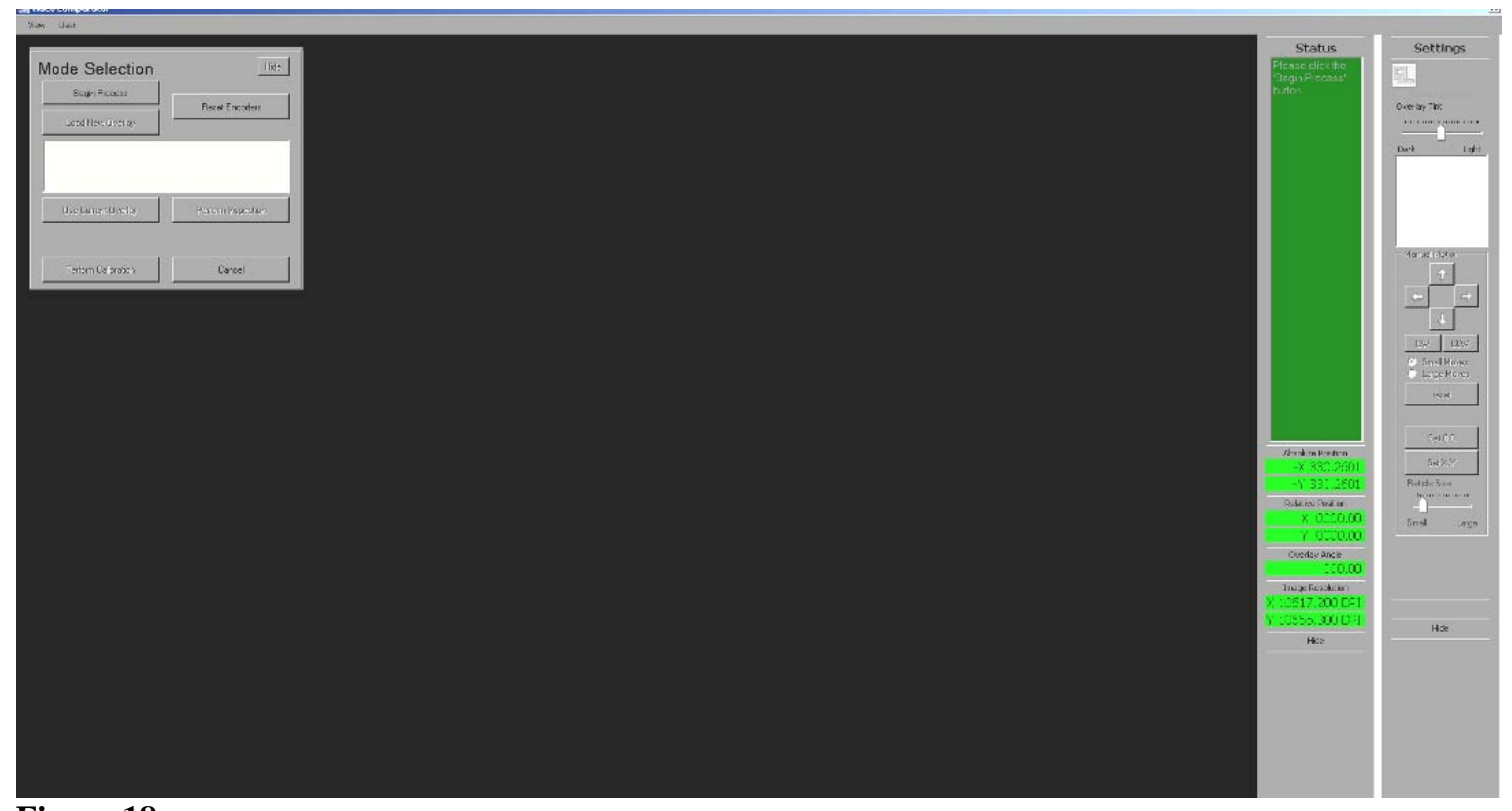

Figure 18

Click the "Begin Process" button in the Mode Selection box to turn on the live image. Watch the prompts in the Status window.

\section{4-B Loading Parts For Inspection}

Place the part to be inspected anywhere on the stage surface. If you are planning to do multiple inspections on identical parts, we recommend using a fixture to repeatedly position the parts accurately. Use the manual Joystick to move the stage so that the origin of the part is within the Field of View of the camera.

\section{4-C Loading Image For Overlay}

This section assumes that an image file has been created following the concepts presented in section 3A.

After fixturing the part to be inspected as described in section 4-B you are ready to load an image for overlay.

1. The first step is to select a size format from the drop-down menu under "Settings", Figure 19. Select "Overlay Size X" and enter the actual size in inches of the converted DXF image. Repeat for "Overlay Size Y".

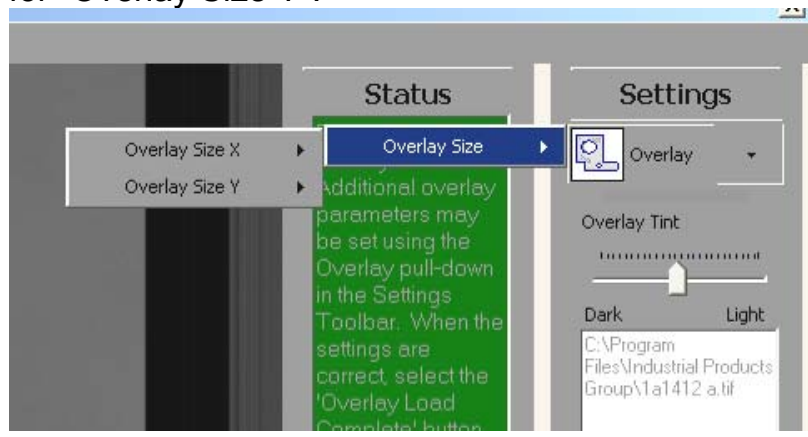

\section{Figure 19}

2. In the Mode Selection box, select "Load New Overlay". Navigate to the image file for the part being inspected. Select the tiff file and click OK. To confirm that the file is the correct size, check 


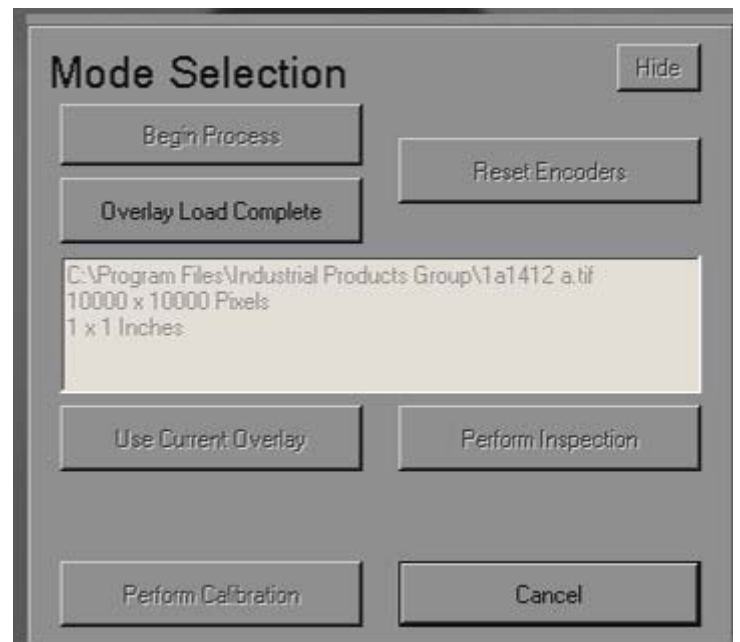

\section{Figure 20}

In the example above, the size of the image is $1 \times 1$ Inches. If this does not correspond to the actual size, go back to Step 1 above and change the "Overlay Size X" and "Overlay Size Y". If the information in this box is correct, click the "Overlay Load Complete" button. Click the "Perform Inspection" button.

3. The overlay image always opens with the lower left-hand corner of the original DXF in the center of the screen, Figure 21.

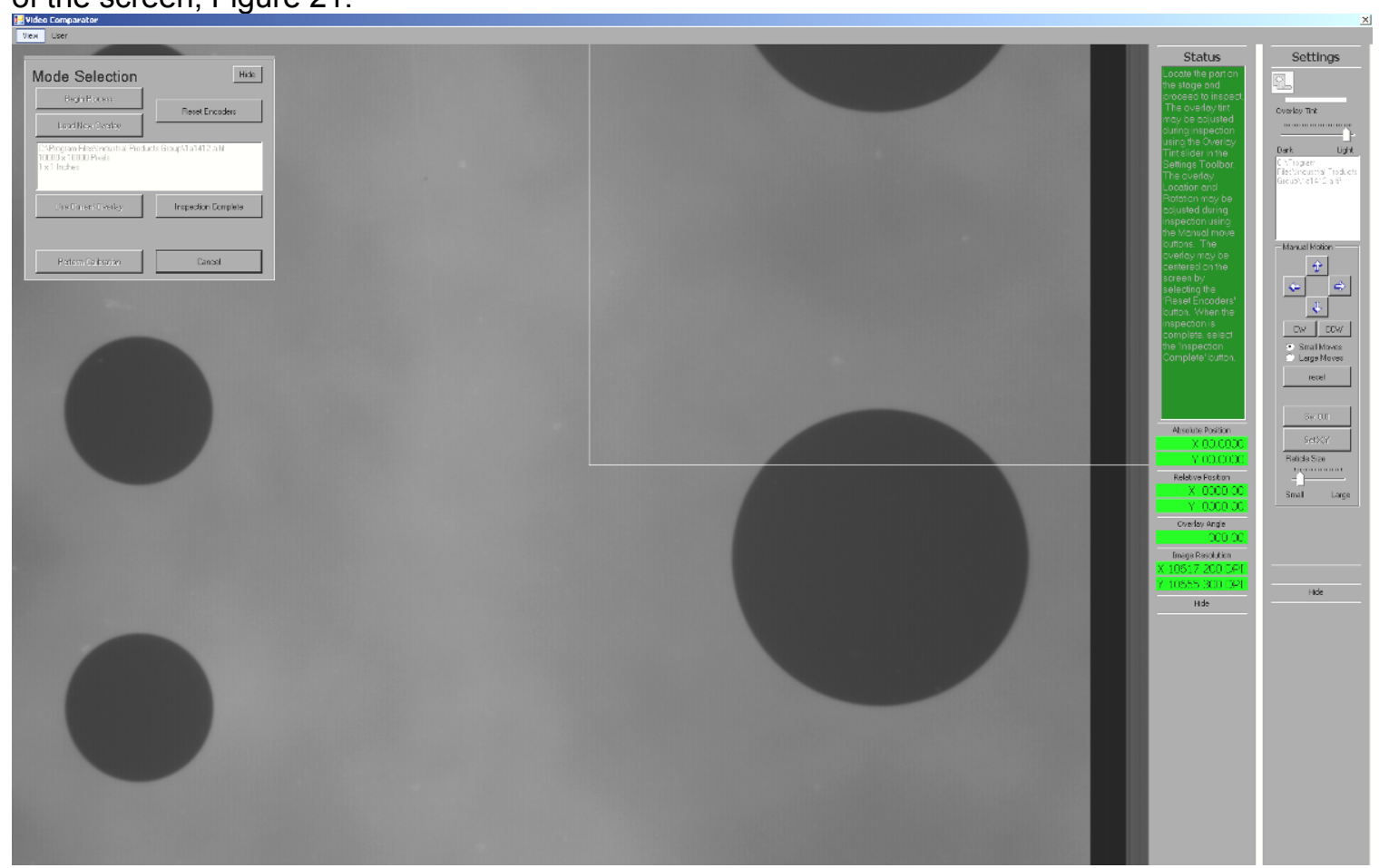

\section{Figure 21}


4. To move the center (or origin) of the overlay to the center of the screen, click "Reset Encoders" in the Mode Selection dialog box, Figure 22.

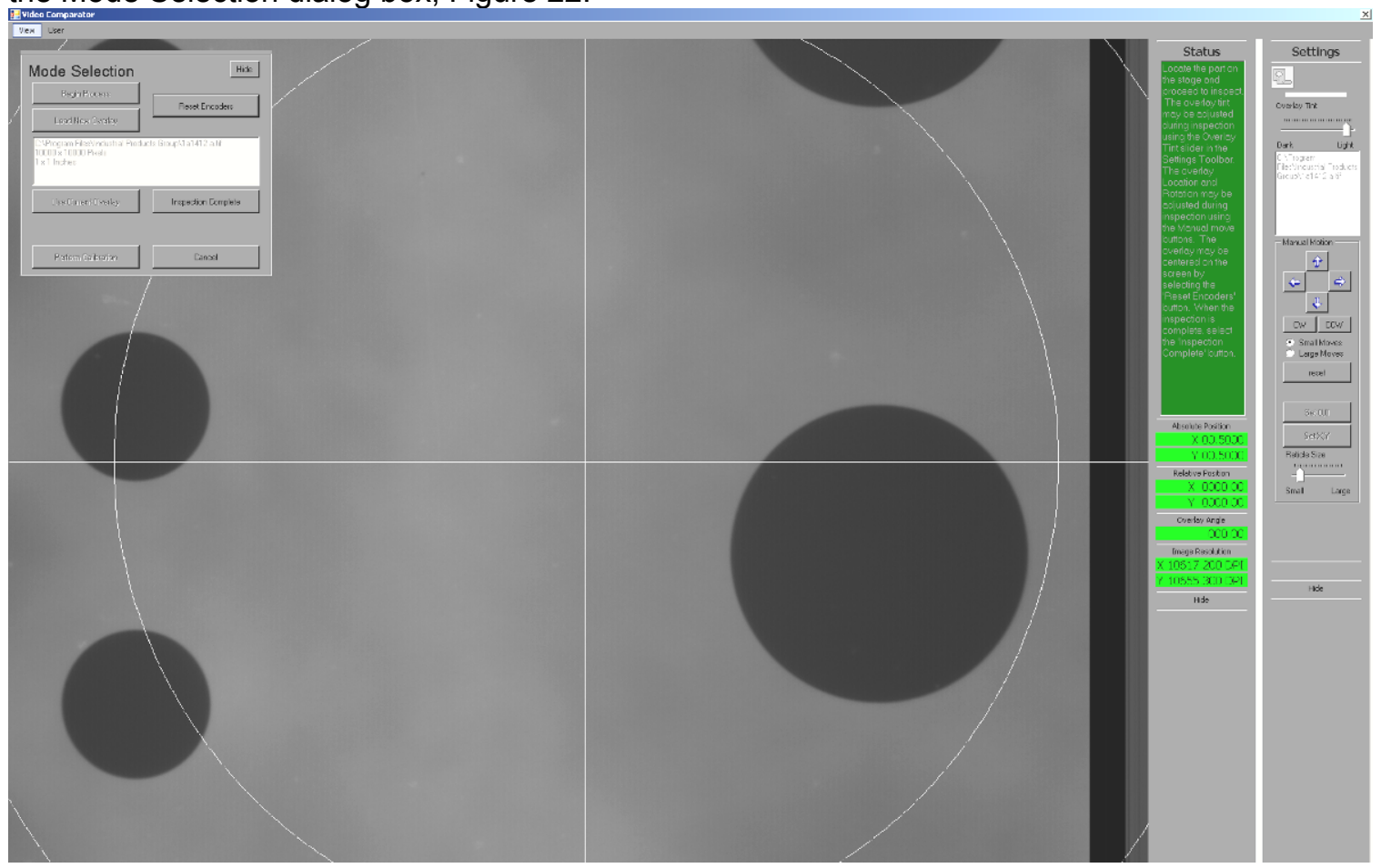

Figure 22

\section{4-D Aligning Part With DXF Overlay}

The procedure for aligning the overlay with the part is as follows:

1. Move the overlay using the arrow buttons on the Manual Motion palette, not the Joystick for the stage, Figure 23.

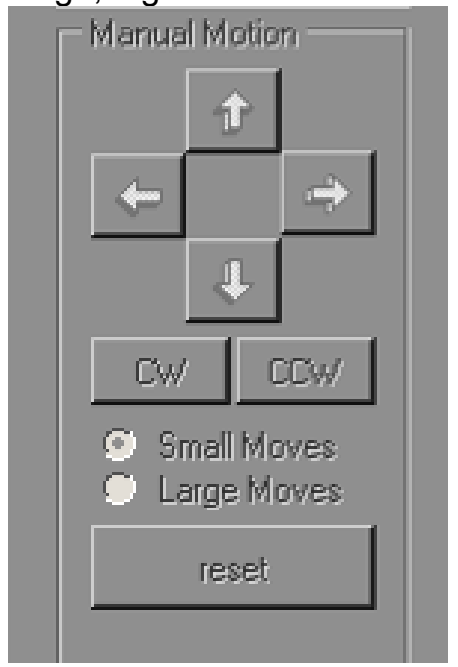

\section{Figure 23}

You may select between small or large moves to adjust how fine or course the overlay image moves for each click of the arrow buttons. 
2. The overlay image can be rotated using the "CW" (clockwise) and "CCW" (counter-clockwise) buttons. It may be easier to center origin of the overlay image over the origin of the live image of the part before rotating the overlay. Once the overlay is positioned in $X$ and $Y$ over the origin of the part, you can move the stage using the joystick so that the feature furthest from the origin is in the field of view. It is now easier to rotate the overlay accurately over the part being inspected.

3. Double check the alignment of the overlay by traversing the stage to different positions on the part. Once you are satisfied the overlay is positioned adequately, your inspection can proceed.

\section{4-E Inspection}

In this section we will go over the general practices for using the video comparator. For general inspections the operator will maneuver the part under the camera using the Joystick while making a visual inspection of the part. If there is a problem seeing the overlay, you have the option to change the overlay tint. Use the slide control on the Settings palette to adjust the overlay tint. Inspection procedure:

1. Use the Joystick to manually traverse the stage to all points of interest of the part under inspection. The speed of the stage can be controlled using the knob directly above the Joystick control. Turn the knob clockwise for faster stage travel

2. You may hide the Mode Selection dialog box by choosing View from the menu and clicking Mode Selection.

3. It is a good practice to return the stage to the origin of the part before completing the inspection.

4. After you are finished inspecting the part, click the "Inspection Complete" button on the Mode Selection dialog box.

5. If you are repeating an inspection of an identical part, click "Use Current Overlay from the Mode Selection dialog box. This will reload the same overlay.

\section{4-F Measurements}

The video comparator has the added capacity to make some basic measurements. As you see on the right hand side of the program window there are $X$ and $Y$ position Readouts. These readouts will automatically set the part origin at zero but you have the ability to set the zero and measure incrementally.

\section{Maintenance}

\section{5-A Cleaning}

Stage:

The video comparator stage is equipped with stepper motors that direct drive a lead screw. The lead screw may require an occasional film of high quality grease. There are covers for both the $\mathrm{X}$-axis and $\mathrm{Y}$-axis the stage. Removing these covers will provide access for lubricating the lead screws and bearings. 


\section{Lens:}

The telecentric lens that the video comparator is equipped with has special lens coatings applied that can be damaged by strong cleaners and oils from exposed skin. To clean use a specially formulated lens cleaner and a lint free cloth.

\section{Monitor:}

Use a soft dry cotton cloth to remove fingerprints and smudges from the screen. If this does not completely remove the dirt and splotches, use a small amount of isopropyl alcohol on the cleaning cloth that has been dampened with water. (A small amount of vinegar can be substituted for the isopropyl alcohol.) Again, spray the liquid on the cloth and never directly onto the screen. Do not use paper towels on an LCD screen as they can scratch the screen. Never use ammonia, ammonia-based cleaners, or strong cleaners, which can cause the surface to yellow and become brittle.

It is good to have a special clean cloth that is dedicated to use on the LCD screen. Don't pick up any old rag, as dirt particles in the rag can scratch the surface of the screen. Special LCD cleaning cloths are available and can be an excellent investment.

\section{5-B Lubrication}

\section{Stage:}

The stage rails require a light coating of thin oil this can be applied with a lint free cloth or a Q-tip works great. Be careful not to leave and debris on the rails such as lint. This can reduce performance of stage.

\section{Camera Bracket:}

The camera bracket requires a light film of oil applied to the linear rail, this can be applied with a lint free cloth or a Q-tip works great. Do not apply oil to the guide shaft part of the camera bracket.

\section{Notes:}




\section{Appendix 2 \\ Computer Code}

D: \VideoComparatorProject -

3\VideoComparatorProject \User Interface \VideoComparator . vb

Public Class VideoComparator

Private Enum eModes

Startup

Star tupcomplete

Homing

HomingComplete

Preparingoverlay

PreparingoverlayComplete

LiveImage

Calibration

CalibrationComplete

Inspection

Inspectioncomplete

Erroroccurred

End Enum

Private Enum eManualMoves

Stopped

MovingDown

Movingup

MovingLeft

MovingRight

RotatingCW

RotatingCCW

End Enum

' Settings Toolstrip variables

Dim bMovingsettingsToolstrip As Boolean

Dim iSettingsLastX As Integer

Dim iSettingsDeltaX As Integer

Dim iSettingsLastY As Integer

Dim isettingsDeltaY As Integer

Dim ioverlayTintTrackBarTopoffset As Integer

Dim ioverlayPathTextboxTopoffset As Integer

Dim iReticleSizeTrackbarTopoffset As Integer

Dim istatusTextboxTopoffset As Integer

' Status Toolstrip variables 
Dim bMovingstatusToolstrip As Boolean

Dim istatuslastX As Integer

Dim istatusdeltaX As Integer

Dim istatuslastY As Integer

Dim istatusdeltaY As Integer

' Overlay Rotate/Translate variables

Dim iTranslateNumberofPixels As Integer

Dim ManualMove As eManualMoves

Private CurrentMode As eModes

Dim Reticle As clsReticle = New clsReticle

Dim Overlay As clsoverlay = New clsoverlay

Dim Camera As clsCamera = New clsCamera

Dim Stepper As clsstepper = New clsstepper

Dim WithEvents QuadBoard As clsquadBoard = New clsQuadBoard

Private Sub VideoComparator_Disposed(ByVal sender As Object, ByVal e As System.

EventArgs) Handles Me.Disposed

My.Settings.Save( )

End Sub

Private Sub VideoComparator_Load(ByVal sender As Object, ByVal e As System. EventArgs)

Handles Me.Load 
D: \VideoComparatorProject -

3\VideoComparatorProject \User Interface \VideoComparator . vb

।

' Sequence of events:

' QuadBoard must be zero'd before loading an overlay.

' An overlay must be loaded before allocating the MILoverlaysized Image

iReticleSizeTrackbarTopoffset $=150$

ioverlayTintTrackBarTopoffset $=280$

iOverlayPathTextboxTopOffset $=350$

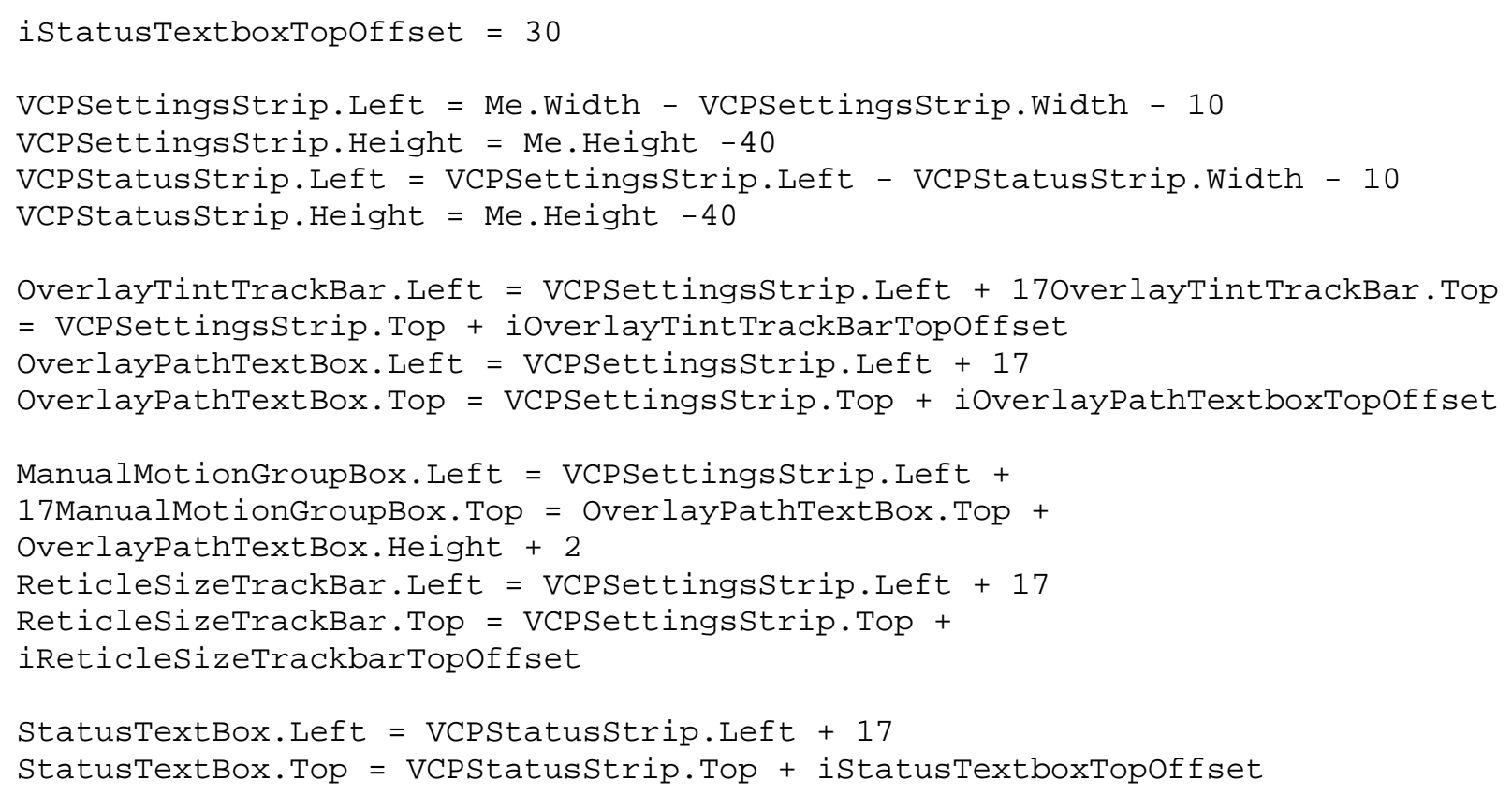

ReticleSizeTrackBar. Minimum = My.Settings.ReticleMinimumSize

ReticleSizeTrackBar. Maximum = My.Settings. ReticleMaximumSize

ReticleSizeTrackBar.Value = My.Settings. ReticleWidth

ReticleSizeLabel.Text = "Reticle Size : " \& ReticleSizeTrackBar.Value

ReticleColorLabel.BackColor = My.Settings.ReticleColor

ReticlePenWidthComboBox.Text = Reticle. ReticlePenWidth

Select Case Reticle.Reticleshape

Case clsReticle.enumReticleshape.Circle

ReticleshapeComboBox. Text = "Circle"

Case clsReticle.enumReticleshape.Square 
ReticleShapeComboBox.Text = "Square"

End Select

Reticle.ReticlePenWidth = My.Settings. ReticlePenWidth

Reticle.ReticleColor = My. Settings. ReticleColor

Reticle.Reticleshape = My. Settings. ReticleShape

Reticle.Reticlewidth = My. Settings. ReticleWidth

Reticle.ReticleHeight $=$ My.Settings. ReticleHeight

QualPinDiameterTextBox.Text = My.Settings.QualificationPinDiameter

CameraSizeXTextBox. Text $=$ My. Settings. CameraSizeX

Camera. CameraSizeX = My. Settings. CameraSizeX

CameraSizeYTextBox. Text = My.Settings. CameraSizeY

Camera. CameraSizeY $=$ My. Settings. CameraSizeY

If Camera.CamerasizeX <= Camera.CameraSizeY Then

Camera. CameraZoom $=$ Math $\cdot$ Round $($ Me.Width / Camera. CameraSizeX, 1)

Else

Camera. CameraZoom = Math. Round (Me.Height / Camera. CameraSizeY, 1)

End If

Camera. CameraResolutionX = My. Settings. CameraResolutionX 
D: \VideoComparatorProject -

3\VideoComparatorProject \User Interface \VideoComparator . vb

Camera. CameraResolutionY $=$ My. Settings. CameraResolutionY

ToolstripResolutionX.Text = Format (Camera.CameraResolutionX, "X 0000.00 DPI")

ToolstripResolutionY.Text = Format (Camera.CameraResolutionY, "Y 0000.00 DPI")

Overlay. OverlayXInches = My. Settings. OverlayInchX

overlay. OverlayYInches $=$

My. Settings. OverlayInchXoverlay. OverlayRotateSourcescale $=$

My.Settings. overlayRotateSourcescale

OverlaySizeComboBox. Text $=$ My.Settings. OverlayInchX

QuadBoard.Encoder InchesPerPulseX = My.Settings.QuadBoardInchesPerCount

QuadBoard.EncoderInchesPerPulseY = My.Settings.QuadBoardInchesPerCount

MILDisplay. Left $=0$

MILDisplay. Top $=0$

MILDisplay. Width = Camera.CameraSizeX * Camera. CameraZoom

MILDisplay. Height = Camera. CameraSizeY * Camera. CameraZoom

MILDisplay.ZoomX = Camera. CameraZoom

MILDisplay. ZoomY = Camera. CameraZoom

MILDisplay. SendToBack( )

Dim pointTemp As New Point

pointTemp. $X=$ MILDisplay.Width $/ 2$

pointTemp.Y = MILDisplay. Height $/ 2$

Reticle.ReticleCenter $=$ pointTemp

Reticle.ReticleCanvas = MILDisplay. Handle

MILImage.SizeX $=$ Me.Width

MILImage. SizeY = Me. Height

MILImage. Allocate( )

MILOver layImage.SizeX = My.Settings. MaxoverlaySizePixels

MILOver layImage. SizeY = My. Settings. MaxoverlaySizePixels

MILOver layImage. Allocate()

MILOver laychunkImage.SizeX = MILImage. SizeX

MILOverlayChunkImage. SizeY $=$ MILImage. SizeY

MILOver layChunkImage. Allocate( )

MILOver layColor Image. SizeX = MILImage. SizeX

MILOver layColor Image. SizeY = MILImage. SizeY

MILOver layColor Image. Allocate()

MILOver layColor Image. Clear (100)

CurrentMode $=$ eModes.Startup

ManualMove $=$ eManualMoves.Stopped 


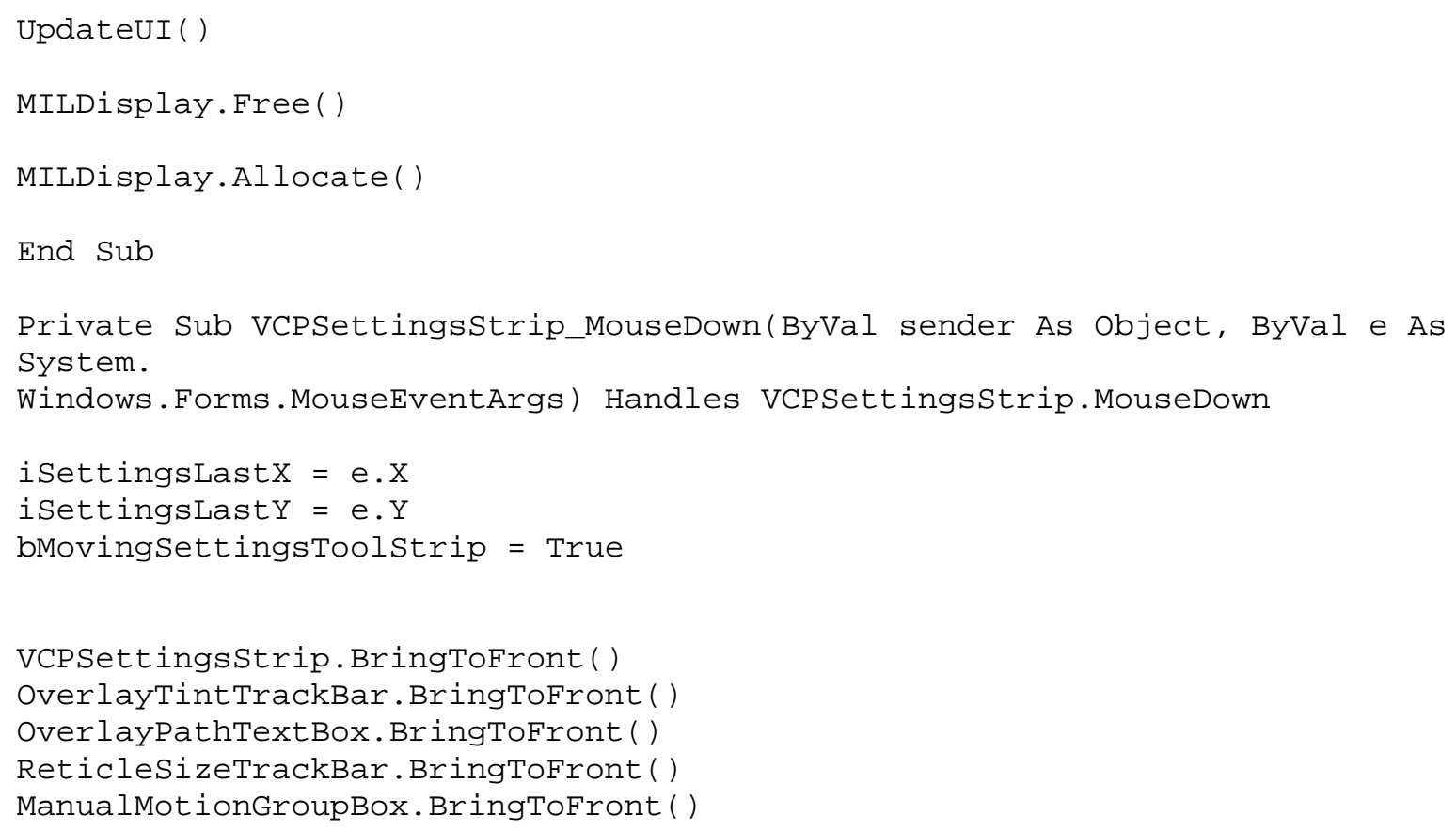


D: \VideoComparatorProject -

3\VideoComparatorProject \User Interface \VideoComparator . vb

End Sub

Private Sub VCPSettingsStrip_MouseLeave(ByVal sender As object, ByVal e As System.

EventArgs) Handles VCPSettingsStrip.MouseLeave

bMovingSettingsToolstrip = False

End Sub

Private Sub VCPSettingsStrip_MouseMove(ByVal sender As object, ByVal e As

System.

Windows.Forms.MouseEventArgs) Handles VCPSettingsStrip.MouseMove

If bMovingsettingsToolstrip Then

iSettingsDeltaX $=$ iSettingsLastX - e.XiSettingsDeltaY $=$ iSettingsLastY - e.Y VCPSettingsStrip.Left = VCPSettingsStrip.Left - iSettingsDeltaX

VCPSettingsStrip.Top = VCPSettingsStrip.Top - iSettingsDeltaY

OverlayTintTrackBar.Left = VCPSettingsStrip. Left + 170verlayTintTrackBar.Top

= VCPSettingsStrip.Top + ioverlayTintTrackBarTopoffset

OverlayPathTextBox. Left $=$ VCPSettingsStrip.Left +17

OverlayPathTextBox.Top = VCPSettingsStrip.Top + ioverlayPathTextboxTopoffset

ManualMotionGroupBox. Left $=$ VCPSettingsStrip. Left +17

ManualMotionGroupBox.Top $=$ OverlayPathTextBox. Top + OverlayPathTextBox. Height

$+2$

ReticleSizeTrackBar.Left = VCPSettingsStrip.Left + 17ReticleSizeTrackBar.Top

= VCPSettingsStrip.Top + iReticleSizeTrackbarTopoffset

End If

End Sub

Private Sub VCPSettingsStrip_MouseUp(ByVal sender As Object, ByVal e As

System. Windows

.Forms.MouseEventArgs) Handles VCPSettingsStrip.Mouseup

bMovingsettingsToolstrip = False

End Sub

Private Sub VCPStatusStrip_MouseDown(ByVal sender As object, ByVal e As System. Windows

.Forms.MouseEventArgs) Handles VCPStatusStrip.MouseDown

iStatuslast $\mathrm{X}=\mathrm{e} \cdot \mathrm{X}$

iStatuslastY $=\mathrm{e} \cdot \mathrm{Y}$

bMovingStatusToolstrip = True

vCPStatusstrip.BringToFront()

StatusTextBox.BringToFront( ) 
End Sub

Private Sub VCPStatusStrip_MouseLeave(ByVal sender As object, ByVal e As System.

EventArgs) Handles VCPStatusStrip.MouseLeave

bMovingstatusToolstrip = False

End Sub

Private Sub VCPStatusStrip_MouseMove(ByVal sender As object, ByVal e As System. Windows

.Forms.MouseEventArgs) Handles VCPStatusStrip.MouseMove

If bMovingstatusToolstrip Then

istatusdeltaX = istatuslastX $-\mathrm{e} \cdot \mathrm{X}$ 
D: \VideoComparatorProject -

3\VideoComparatorProject \User Interface \VideoComparator . vb

istatusdeltaY = istatuslastY - e.Y

VCPStatusStrip.Left = VCPStatusStrip.Left - iStatusdeltaXVCPStatusStrip.Top = VCPStatusStrip.Top - istatusdeltaY

StatusTextBox. Left = VCPStatusStrip. Left + 17StatusTextBox.Top $=$

VCPStatusStrip.Top + istatusTextboxTopoffset

End If

End Sub

Private Sub VCPStatusStrip_MouseUp(ByVal sender As Object, ByVal e As

System. Windows.

Forms.MouseEventArgs) Handles VCPStatusStrip.MouseUp

bMovingstatusToolstrip = False

End Sub

Private Sub ReticleColorMenuItem_Click(ByVal sender As System.Object, ByVal e As

System.EventArgs) Handles ReticleColorMenuItem.Click

If VCPColorDialog. ShowDialog ()$=$ Windows.Forms.DialogResult.OK Then

ReticleColor Label. BackColor = VCPColorDialog. Color

Reticle.ReticleColor = VCPColorDialog.Color

End If

End Sub

Private Sub SettingsToolstripMenuItem_Click(ByVal sender As System.object, Byval e As

System.EventArgs) Handles SettingsToolstripMenuItem.Click

VCPSettingsStrip.Visible $=$ SettingsToolstripMenuItem. Checked OverlayPathTextBox.Visible = SettingsToolstripMenuItem. Checked

ReticleSizeTrackBar.Visible = SettingsToolstripMenuItem. Checked

End Sub

Private Sub SettingsCloseButton_Click(ByVal sender As System.Object, ByVal e As System.EventArgs) Handles SettingsCloseButton.Click

VCPSettingsstrip.Visible $=$ False

OverlayPathTextBox.Visible = False

ReticleSizeTrackBar.Visible = False

SettingsToolstripMenuItem. Checked = False

End Sub

Private Sub StatusToolstripMenuItem_Click(ByVal sender As System.object, Byval e As

System.EventArgs) Handles StatusToolstripMenuItem.Click 
VCPStatusStrip.Visible = StatusToolstripMenuItem. Checked

End Sub

Private Sub StatusCloseButton_Click(ByVal sender As System.Object, ByVal e As System.

EventArgs) Handles StatusCloseButton.Click

VCPStatusStrip.Visible $=$ False

StatusToolstripMenuItem. Checked $=$ False

End Sub

Private Sub ReticleColorLabel_Click(ByVal sender As System.Object, ByVal e As System.

EventArgs) Handles ReticleColorLabel.Click

If VCPColorDialog. ShowDialog ()$=$ Windows.Forms.DialogResult.OK Then 
D: \VideoComparatorProject -

3\VideoComparatorProject \User Interface \VideoComparator . vb

ReticleColor Label.BackColor = VCPColorDialog.Color

Reticle.ReticleColor = VCPColorDialog.Color

End If

End Sub

Private Sub ReticleShapeComboBox_TextChanged(ByVal sender As Object, ByVal e As System.EventArgs) Handles ReticleShapeComboBox.TextChanged

Select Case ReticleShapeComboBox.Text

Case "Circle"

Reticle. Reticleshape $=$ clsReticle $\cdot$ enumReticleShape . Circle

Case "Square"

Reticle.ReticleShape $=$ clsReticle.enumReticleShape. Square

End Select

End Sub

Private Sub ReticlePenWidthComboBox_TextChanged(ByVal sender As Object, ByVal e As

System.EventArgs) Handles ReticlePenWidthComboBox.TextChanged

Reticle.ReticlePenWidth = CInt (ReticlePenWidthComboBox.Text)

End Sub

Private Sub ReticleSizeTrackBar_Scroll(ByVal sender As System.object, ByVal e As

System.EventArgs) Handles ReticleSizeTrackBar.Scroll

ReticleSizeLabel.Text = "Reticle Size : " \& ReticleSizeTrackBar.Value Reticle.ReticleHeight $=$ ReticleSizeTrackBar.ValueReticle. Reticlewidth $=$ ReticlesizeTrackBar. Value

End Sub

Private Sub QualPinDiameterTextBox_TextChanged(ByVal sender As Object, ByVal e As

System.EventArgs) Handles QualPinDiameterTextBox.TextChanged

If IsNumeric(QualPinDiameterTextBox.Text) Then

Reticle.ReticlePinDiameter = QualPinDiameterTextBox.Text

Else

QualPinDiameterTextBox.Text $=$ My.Settings.QualificationPinDiameter 


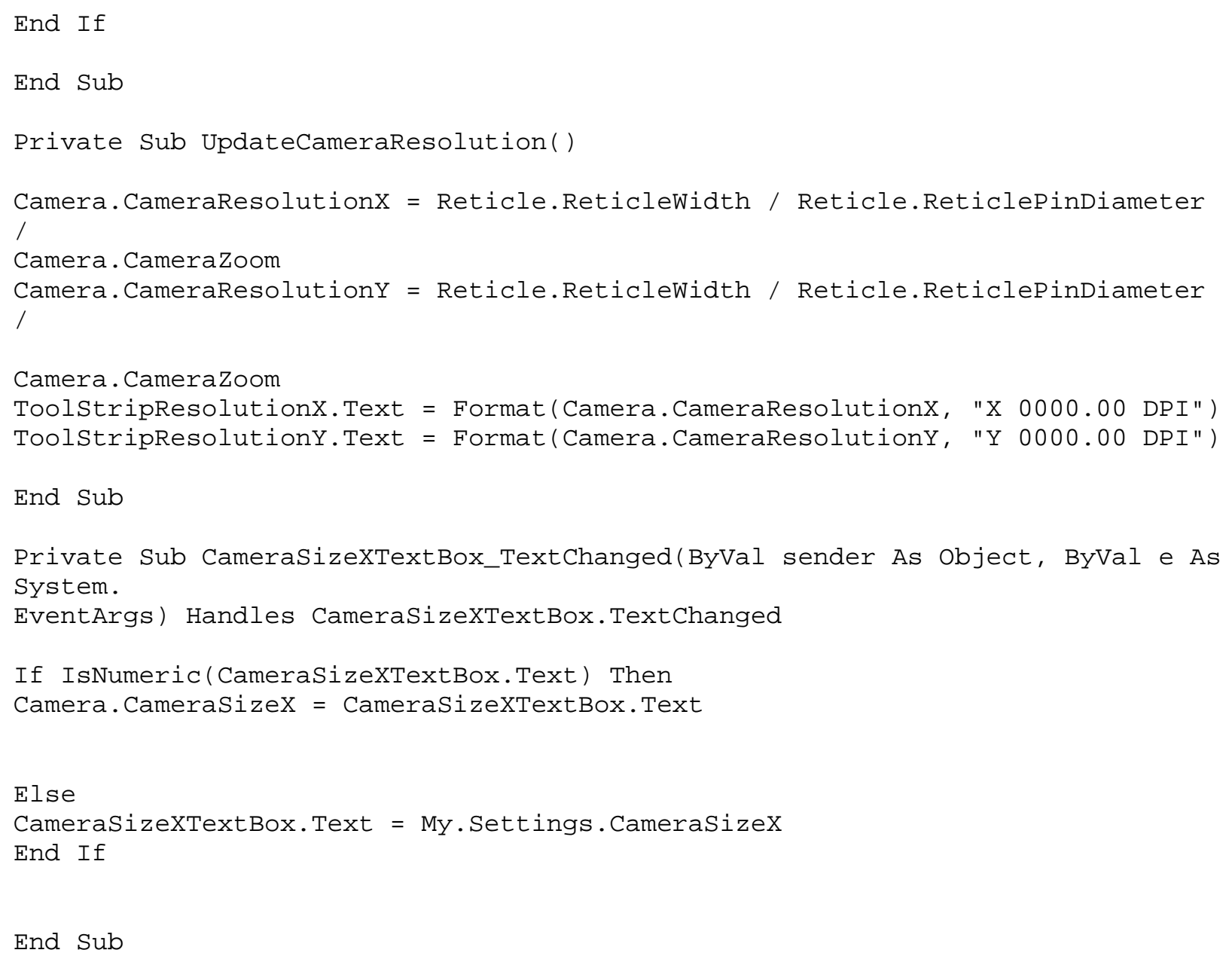


D: \VideoComparatorProject -

3\VideoComparatorProject \User Interface \VideoComparator . vb

Private Sub CameraSizeYTextBox_TextChanged(ByVal sender As Object, ByVal e As System.

EventArgs) Handles CameraSizeYTextBox.TextChanged

If IsNumeric(CameraSizeYTextBox.Text) Then

Camera $\cdot$ CameraSizeY $=$ CameraSizeYTextBox $\cdot$ Text

Else

CameraSizeYTextBox. Text $=$ My.Settings. CameraSizeY

End If

End Sub

Private Sub QuadBoard_Counter1Changed() Handles QuadBoard.Counter1Changed

ToolstripAbsoluteY.Text = Format (QuadBoard.Counter1Inches, "Y $00.0000 "$ ") If CurrentMode = eModes. Inspection Then Overlay. MoveToInches (QuadBoard. Counter2Inches, QuadBoard.Counter1Inches)

End Sub

Private Sub QuadBoard_Counter2Changed() Handles QuadBoard.Counter2Changed

ToolstripAbsoluteX.Text = Format (QuadBoard. Counter2Inches, "X $\odot \odot .0000 ")$ If CurrentMode $=$ eModes. Inspection Then Overlay. MoveToInches (QuadBoard. Counter2Inches, QuadBoard.Counter1Inches)

End Sub

Private Sub UpdateMILOverlayChunk()

MILOver layColor Image. Clear (Over lay. Over layColor)

MILOver laySizedImage.CopyRegion(MILOver layImage, Matrox. ActiveMIL. ImBandConstants .

imAllBands,

Overlay. OverlayRotateRect.X, Overlay.

OverlayRotateRect.Y,

Matrox. ActiveMIL. ImBandConstants.imAllBands, $\odot$

, $\Theta$, -

Overlāy.overlayRotateRect.Width, Overlay.

OverlayRotateRect. Height)

MILOver layRotateImageProcessing.Rotate(Over lay. Over layAngle)

MILOver layReSizedImage. CopyRegion(MILOver laySizedAndRotatedImage, Matrox.ActiveMIL

. ImBandConstants.imAllBands, - 
Overlay. OverlayRotateSourceXBorder - 1, Overlay.

OverlayRotateSourceYBorder - 1, -

Matrox.ActiveMIL. ImBandConstants.imAllBands, $\odot$,

0,

Over lay.OverlaySourceRect.Width, Overlay.

OverlaySourceRect. Height)

MILOver layScaleImageProcessing. Resize(Overlay. ImageTo0verlayScalex, overlay. ImageToover layscaleY, Matrox. ActiveMIL. ImageProcessing.

ImpInterpolationModeConstants.impInterpolatedResize, Matrox. ActiveMIL. ImageProcessing.

ImpoverscanModeConstants. impoverscanDisable)

End Sub

Private Sub ReallocateMILOverlayChunk()

If MILOverlayReSizedImage. IsAllocated Then

MILOverlayReSizedImage. Free( )

MILOver layReSizedImage.SizeX = (Camera. CameraSizeX * Overlay .

Over layToImageScaleX) +1 
D: \VideoComparatorProject -

3\VideoComparatorProject \User Interface \VideoComparator . vb

MILOver layReSizedImage.SizeY = (Camera. CameraSizeY * Overlay.

Over layToImageScaleY) +1

MILOver layReSizedImage. Allocate( )

End If

If MILOverlaysizedAndRotatedImage. IsAllocated Then

MILOver laysizedAndRotatedImage. Free( )

MILOverlaySizedAndRotatedImage.SizeX = overlay.overlayRotateSourceScale *

(Camera.CameraSizeX * Overlay.overlayToImageScaleX) +1

MILOver laySizedAndRotatedImage.SizeY = Overlay. OverlayRotateSourcescale * (Camera.CameraSizeY * Overlay.overlayToImageScaleY) + 1

MILOver laySizedAndRotatedImage. Allocate( )

End If

If MILOverlaySizedImage. IsAllocated Then

MILOver laysizedImage. Free( )

MILOverlaySizedImage. SizeX = Overlay.overlayRotateSourceScale * (Camera.

CameraSizeX * Overlay. Over layToImageScaleX) +1

MILOver laysizedImage.SizeY = Overlay.OverlayRotateSourceScale * (Camera.

CameraSizeY * Overlay. OverlayToImageScaleY) +1

MILOver laySizedImage. Allocate( )

End If

End Sub

Private Sub MILDigitizer_GrabFrameEnd(ByVal sender As Object, ByVal e As System.

EventArgs) Handles MILDigitizer.GrabFrameEnd

Select Case CurrentMode

Case eModes.Calibration

Reticle.Draw()

UpdateCameraResolution( )

Try

MILDigitizer.Grab( )

Catch ex As ExceptionstatusTextBox.Text = "Digitizer Failed. Please confirm camera is

powered on and all cables are correctly connected."

CurrentMode $=$ eModes. ErrorOccurred

UpdateUI ( )

Finally 


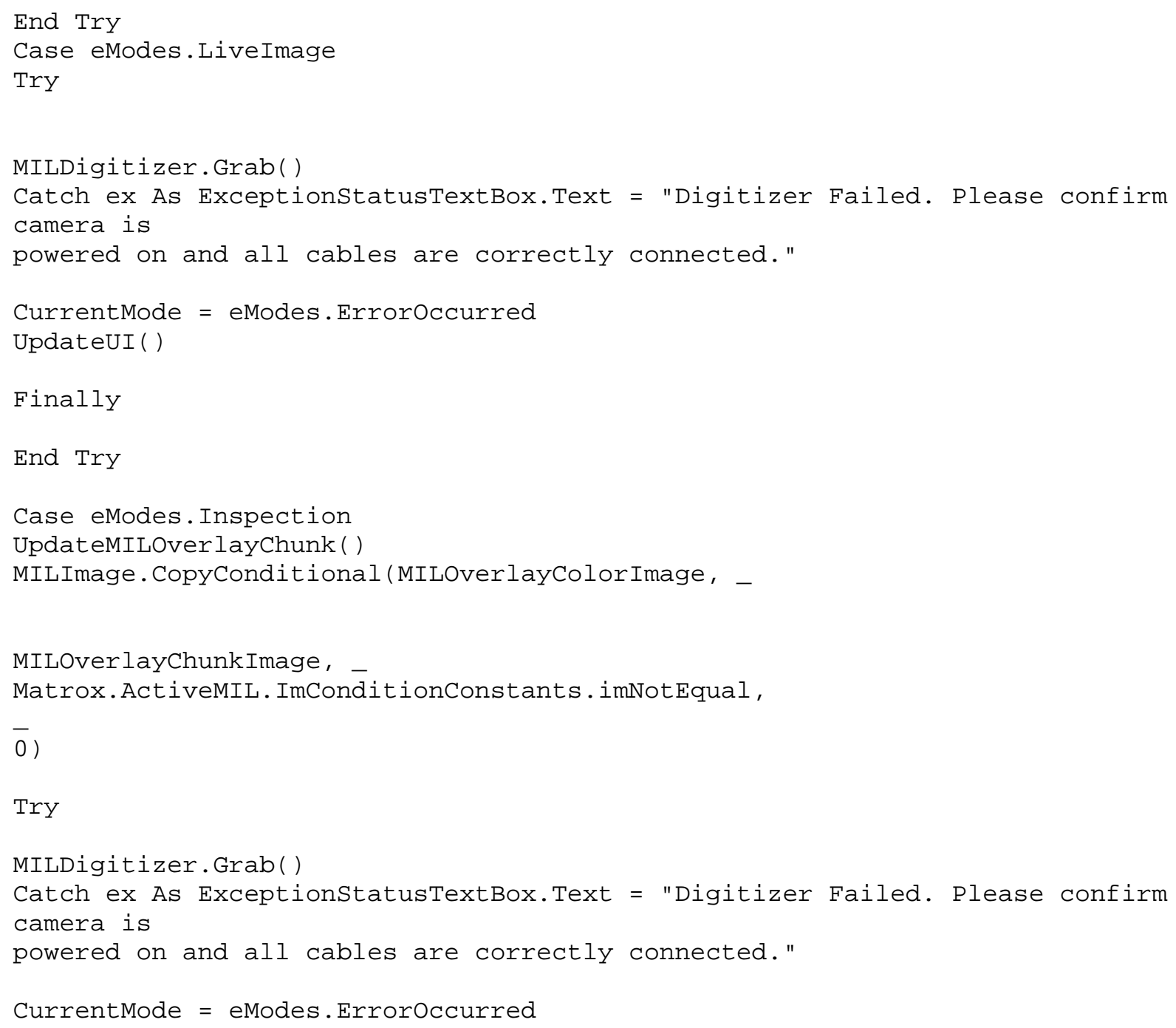


D: \VideoComparatorProject -

3\VideoComparatorProject \User Interface \VideoComparator . vb

UpdateUI( )

Finally

End Try

End Select

End Sub

Private Sub ResetQuadBoardButton_Click(ByVal sender As System.Object, ByVal e As

System.EventArgs) Handles ResetQuadBoardButton.Click

CurrentMode $=$ eModes. Homing

UpdateUI( )

QuadBoard.Initialize( )

CurrentMode $=$ eModes. HomingComplete

UpdateUI( )

End Sub

Private Sub LoadOverlayButton_Click(ByVal sender As System.Object, ByVal e As System.

EventArgs) Handles LoadOverlayButton.Click

If CurrentMode $=$ eModes.Preparingoverlay Then

Savesettings( )

CurrentMode $=$ eModes. Preparingover layComplete

UpdateUI( )

Else

CurrentMode = eModes. Preparingoverlay

UpdateUI( )

VCPOpenDialog.Filter $=$ "image files|*.bmp; *.tif; *.jpg"

VCPOpenDialog.ShowDialog( )

If VCPOpenDialog. FileName <> "" Then

Overlay. OverlayPath $=$ VCPOpenDialog. FileNameOverlayPathTextBox. Text $=$ Over lay. Over layPathMILOver layImage.Clear( )

MILOver layImage. Load(Over lay. Over layPath, True)

Overlayupdate( )

End If

End If

End Sub 
Private Sub UseExistingoverlayButton_Click(ByVal sender As System.Object, Byval e As

System.EventArgs) Handles UseExistingOverlayButton.Click

Savesettings()

CurrentMode $=$ eModes. Preparingover layComplete

UpdateUI( )

End Sub

Private Sub LiveImageButton_Click(ByVal sender As System.object, ByVal e As System.

EventArgs) Handles LiveImageButton.Click

If CurrentMode $=$ eModes. LiveImage Then

CurrentMode $=$ eModes. StartupComplete

UpdateUI( )

Else

CurrentMode $=$ eModes. LiveImage 
D: \VideoComparatorProject -

3\VideoComparatorProject \User Interface \VideoComparator . vb

UpdateUI( )

Try

MILDigitizer.Grab( )

Catch ex As ExceptionstatusTextBox.Text = "Digitizer Failed. Please confirm camera is poweredon and all cables are correctly connected."

CurrentMode $=$ eModes. Erroroccurred

UpdateUI( )

Finally

End Try

End If

End Sub

Private Sub Overlayupdate()

OverlayParametersTextBox.Clear ()

OverlayParametersTextBox.Text = overlay.overlayPath \& vbcrLf

OverlayParametersTextBox.Text $=$ overlayParametersTextBox.Text \&

MILOver layImage.

SizeX \& " $x$ " \& MILOverlayImage.SizeY \& " Pixels" \& vbcrLf

OverlayParametersTextBox.Text $=$ overlayParametersTextBox. Text \&

OverlaySizeComboBox. Text \& " x " \& OverlaySizeComboBox. Text \& " Inches" \& vbCrLf

End Sub

Private Sub UpdateuI()

CameraToolstripButton. Enabled = False

ReticleToolstripButton. Enabled = False

ReticleColorLabel. Enabled = False

ReticleSizeTrackBar. Enabled = False

OverlayToolstripButton. Enabled = False

OverlayColorLabel. Enabled = False

OverlayTintTrackBar. Enabled = False

ManualMotionGroupBox. Enabled $=$ False

ModePanelHideButton. Enabled = False

ResetModeButton. Enabled = False

UseExistingOverlayButton. Enabled = False

ResetQuadBoardButton. Enabled $=$ False

ResetQuadBoardButton. Text $=$ "Reset/Home Encoders"

LoadOverlayButton. Enabled = False

LoadOverlayButton. Text = "Load Overlay"

LiveImageButton. Enabled $=$ False 
LiveImageButton.Text = "Begin Live Image Capture"

CalibrateButton. Enabled = False

CalibrateButton. Text = "Perform Calibration"

InspectButton. Enabled = False

InspectButton. Text = "Perform Inspection"

Select Case CurrentMode

Case eModes.Calibration

StatusTextBox.Text = "Locate the calibration pin on the stage and proceed to calibrate. " \&

"Calibration settings my be adjusted using the Camera

and Reticle pull-downs in the Settings Toolbar. " \& -

"When the Calibration is complete, select the

'Calibration Complete' button."

StatusTextBox.BackColor $=$ Color. DarkGreen

CalibrateButton. Text = "Calibration Complete"

CalibrateButton. Enabled = True

CameraToolstripButton. Enabled = True

ReticleToolstripButton. Enabled = True

ReticleColorLabel. Enabled = True

ReticleSizeTrackBar. Enabled = True 
D: \VideoComparatorProject -

3\VideoComparatorProject \User Interface \VideoComparator . vb

Case eModes.CalibrationComplete

StatusTextBox.Text $=$ "Please select 'Reset/Home Encoders', 'Load New Overlay', 'Use Current Overlay' (if available), 'View Live Image', or 'Calibrate

Camera'"

StatusTextBox.BackColor = Color. DarkGreen

ResetQuadBoardButton. Enabled = True

LoadOverlayButton. Enabled = True

LiveImageButton. Enabled = True

CalibrateButton. Enabled = True

If Overlay.OverlayPath <> "" Then

UseExistingoverlayButton. Enabled = True

End If

Case eModes.Homing

Case eModes.HomingComplete

StatusTextBox.Text $=$ "Please select 'Reset/Home Encoders', 'Load New Overlay', 'Use Current Overlay' (if available), 'View Live Image', or 'Calibrate

Camera'"

StatusTextBox.BackColor = Color. DarkGreen

ResetQuadBoardButton. Enabled = True

LoadoverlayButton. Enabled = True

LiveImageButton. Enabled = True

CalibrateButton. Enabled = True

If Overlay. OverlayPath $<>$ " Then

UseExistingoverlayButton. Enabled = True

End If

Case eModes. InspectionStatusTextBox.Text $=$ "Locate the part on the stage and proceed to inspect.

"\&

"The overlay tint may be adjusted during inspection

using the Overlay Tint slider in the Settings Toolbar. " \&

"When the inspection is complete, select the

'Inspection Complete' button."

StatusTextBox.BackColor $=$ Color $\cdot$ DarkGreen

InspectButton. Text = "Inspection Complete"

InspectButton. Enabled = True

ManualMotionGroupBox. Enabled = True

ManualMoveRepeatTimer. Enabled = True

OverlayTintTrackBar. Enabled = True

ModePanelHideButton. Enabled = True 


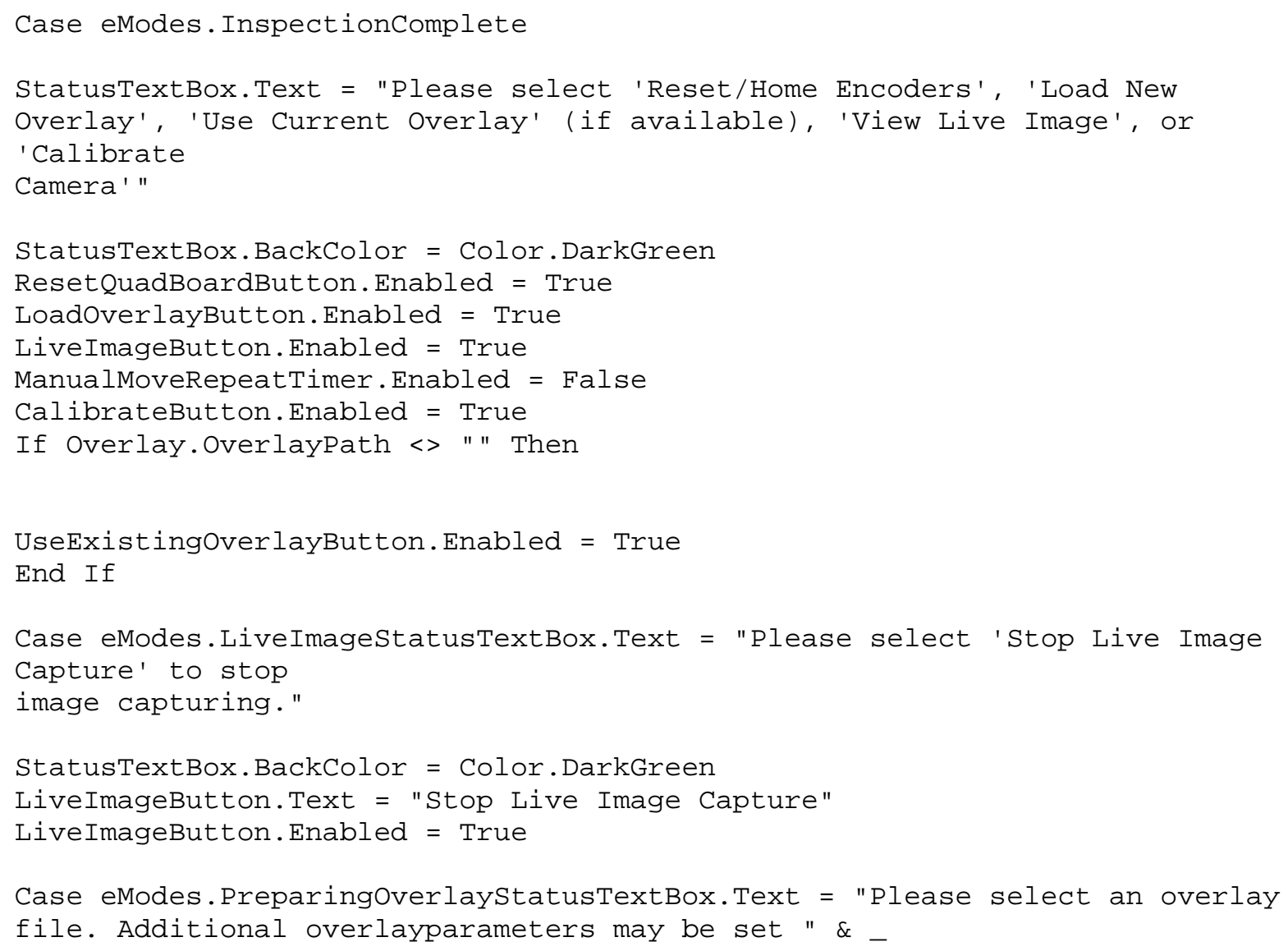


D: \VideoComparatorProject -

3\VideoComparatorProject \User Interface \VideoComparator . vb

"using the Overlay pull-down in the settings Toolbar.

"\&

"When the settings are correct, select the 'Overlay

Load Complete' button."

StatusTextBox.BackColor $=$ Color. DarkGreen

LoadOverlayButton. Text = "Overlay Load Complete"

LoadOverlayButton. Enabled = True

OverlayToolstripButton. Enabled = True

OverlayTintTrackBar. Enabled = True

Case eModes.PreparingoverlayCompletestatusTextBox. Text $=$ "Please select the 'Perform Inspection' button"

StatusTextBox.BackColor = Color. DarkGreen

InspectButton. Enabled = True

Case eModes.StartupstatusTextBox.Text $=$ "Please Home the stage. When complete, click the

'Reset/Home Encoders' button"

StatusTextBox.BackColor $=$ Color. DarkGreen

ResetQuadBoardButton. Enabled = True

Case eModes.StartupComplete

StatusTextBox.Text = "Please select 'Reset/Home Encoders', 'Load New Overlay', 'Use Current Overlay' (if available), 'View Live Image', or 'Calibrate

Camera'"

StatusTextBox.BackColor = Color. DarkGreen

ResetQuadBoardButton. Enabled = True

LoadOver layButton. Enabled = True

LiveImageButton. Enabled = True

CalibrateButton. Enabled = True

If Overlay.OverlayPath <> " "Then

UseExistingOverlayButton. Enabled $=$ True

End If

Case eModes.ErrorOccurred

StatusTextBox. BackColor = Color. Yellow

ManualMoveRepeatTimer. Enabled = False

ResetQuadBoardButton. Enabled = True

ResetModeButton. Enabled = True

End Select

End Sub 
Private Sub ModeSelectionToolstripMenuItem_Click(ByVal sender As System.Object, ByVale As System.EventArgs) Handles

ModeSelectionToolstripMenuItem.Click

ToDoGroupBox.Visible $=$ ModeSelectionToolstripMenuItem. Checked

End Sub

Private Sub ResetModeButton_Click(ByVal sender As System.object, ByVal e As System.

EventArgs) Handles ResetModeButton.Click

Overlay.overlaySourceoffsetXInches $=0$

Overlay. OverlaySourceoffsetYInches $=0$

Overlay. OverlayAngle $=0$

Overlay. OverlayPath $=" "$

OverlayPathTextBox.Text = overlay.overlayPath

CurrentMode $=$ eModes. StartupComplete

UpdateUI ( )

End Sub

Private Sub CalibrateButton_Click(ByVal sender As System.Object, ByVal e As System.

EventArgs) Handles CalibrateButton.Click

If CurrentMode $=$ eModes . Calibration Then 
D: \VideoComparatorProject -

3\VideoComparatorProject \User Interface \VideoComparator . vb

Savesettings( )

CurrentMode $=$ eModes. CalibrationComplete

UpdateUI( )

Else

CurrentMode $=$ eModes . Calibration

UpdateUI ( )

Try

MILDigitizer.Grab( )

Catch ex As ExceptionstatusTextBox.Text = "Digitizer Failed. Please confirm camera is poweredon and all cables are correctly connected."

CurrentMode $=$ eModes. ErrorOccurred

UpdateUI ( )

Finally

End Try

End If

End Sub

Private Sub InspectButton_Click(ByVal sender As System.Object, ByVal e As System.

EventArgs) Handles InspectButton.Click

If CurrentMode $=$ eModes. Inspection Then

CurrentMode $=$ eModes. InspectionComplete

UpdateUI( )

Else

CurrentMode $=$ eModes. Inspection

UpdateUI( )

Overlay. OverlayXPixels = MILOverlayImage.SizeX

overlay. OverlayYPixels = MILOverlayImage.SizeY

Overlay. OverlayXInches $=$ OverlaySizeComboBox. Text

Overlay. OverlayYInches $=$ OverlaySizeComboBox. Text

Overlay. ImageDPIX = Camera. CameraResolutionX

overlay. ImageDPIY = Camera. CameraResolutionY

Overlay. ImageXPixels = Camera. CameraSizeX

overlay. ImageYPixels = Camera. CameraSizeY

Overlay.overlayColor = OverlayTintTrackBar.Value

Overlay.UpdateoverlayParameters( )

ReallocateMILOverlayChunk() 
MILDigitizer.Grab( )

End If

End Sub

Private Sub SaveSettings()

My. Settings. CameraSizeX $=$ Camera. CameraSizeX

My. Settings. CameraSizeY = Camera. CameraSizeY

My.Settings. OverlayInchX = Overlay.overlayXInches

My.Settings. OverlayColor = Overlay. OverlayColor

My.Settings. CameraResolutionX = Camera. CameraResolutionX

My.Settings. CameraResolutionY = Camera.CameraResolutionY

My.Settings.QualificationPinDiameter = Reticle. ReticlePinDiameter

My.Settings. Reticlewidth = Reticle. ReticleWidth

My. Settings. ReticleHeight $=$ Reticle. ReticleHeight

My.Settings.ReticlePenWidth = Reticle.ReticlePenWidth

My.Settings. Reticleshape $=$ Reticle. ReticleShape

My.Settings.ReticleColor $=$ Reticle.ReticleColor

My.Settings.Save( )

End Sub 
D: \VideoComparatorProject -

3\VideoComparatorProject \User Interface \VideoComparator . vb

Private Sub LoadSettings()

,

End Sub

Private Sub UpdateoverlayoffsetDisplay()

ToolstripRelativeX.Text = Format (Overlay. OverlaySourceoffsetXInches, "X $00.000 "$ )

ToolstripRelativeY.Text = Format (Overlay. OverlaySource0ffsetYInches, "Y $00.000 "$ )

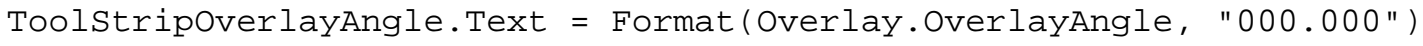

End Sub

Private Sub OverlaySizeComboBox_TextChanged(ByVal sender As Object, ByVal e As System.

EventArgs) Handles OverlaySizeComboBox.TextChanged

If CurrentMode $=$ eModes. Preparingoverlay Then

Overlay. OverlayXInches = OverlaySizeComboBox. Text

overlay. OverlayYInches $=$ OverlaySizeComboBox. Text

Overlayupdate( )

End If

End Sub

Private Sub UpButton_MouseDown(ByVal sender As object, ByVal e As System. Windows. Forms

. MouseEventArgs) Handles UpButton.MouseDown

ManualMove = eManualMoves. Movingup

Updateover layoffsetDisplay( )

End Sub

Private Sub UpButton_MouseUp(ByVal sender As object, ByVal e As System. Windows. Forms.

MouseEventArgs) Handles UpButton.MouseUpManualMove = eManualMoves.Stopped

End Sub

Private Sub LeftButton_MouseDown(ByVal sender As Object, ByVal e As System. Windows.

Forms. MouseEventArgs) Handles LeftButton. MouseDown

ManualMove $=$ eManualMoves. MovingLeft

UpdateoverlayoffsetDisplay( ) 
End Sub

Private Sub LeftButton_Mouseup(ByVal sender As object, ByVal e As System. Windows. Forms . MouseEventArgs) Handles LeftButton.MouseUp

ManualMove $=$ eManualMoves.Stopped

End Sub

Private Sub DownButton_MouseDown(ByVal sender As object, ByVal e As System. Windows.

Forms. MouseEventArgs) Handles DownButton. MouseDown

ManualMove = eManualMoves. MovingDown

Updateover layoffsetDisplay( )

End Sub

Private Sub DownButton_Mouseup(ByVal sender As Object, ByVal e As System. Windows. Forms . MouseEventArgs) Handles DownButton. MouseUp

ManualMove $=$ eManualMoves.Stopped

End Sub

Private Sub RightButton_MouseDown(ByVal sender As object, ByVal e As System.Windows.

Forms.MouseEventArgs) Handles RightButton. MouseDown

ManualMove = eManualMoves. MovingRight

UpdateoverlayoffsetDisplay( )

End Sub

Private Sub RightButton_Mouseup(ByVal sender As object, ByVal e As System. Windows. Forms.MouseEventArgs) Handles RightButton. MouseUpManualMove = eManualMoves.Stopped

End Sub

Private Sub SmallMovesRadioButton_CheckedChanged(ByVal sender As System.Object, ByVale As System.EventArgs) Handles

SmallMovesRadioButton. CheckedChangediTranslateNumberofPixels = My.Settings.overlayTranslateSmallMoveEnd Sub 
D: \VideoComparatorProject -

3\VideoComparatorProject \User Interface \VideoComparator . vb

Private Sub LargeMovesRadioButton_CheckedChanged(ByVal sender As

System.Object, ByVale As System.EventArgs) Handles

LargeMovesRadioButton. CheckedChangediTranslateNumberofPixels =

My.Settings.overlayTranslateLargeMoveEnd Sub

Private Sub ResetButton_Click(ByVal sender As System.Object, ByVal e As

System.

EventArgs) Handles ResetButton.Click

Overlay. OverlaySourceoffsetXInches $=0$

Overlay. OverlaySourceoffsetYInches $=0$

UpdateoverlayoffsetDisplay( )

End Sub

Private Sub ManualMoveRepeatTimer_Tick(ByVal sender As object, ByVal e As System.

EventArgs) Handles ManualMoveRepeatTimer.Tick

Select Case ManualMove

Case eManualMoves. MovingDownover lay. MoveDeltaPixels ( $\odot$,

iTranslateNumberofPixels)

UpdateoverlayoffsetDisplay( )

Case eManualMoves.MovingLeftover lay.MoveDeltaPixels(iTranslateNumberOfPixels, $\odot)$ UpdateoverlayoffsetDisplay( )

Case eManualMoves. MovingRightoverlay. MoveDeltaPixels(-

iTranslateNumberOfPixels, $\odot$ )

UpdateoverlayoffsetDisplay( )

Case eManualMoves.Movingupoverlay.MovedeltaPixels( $\odot$, -

iTranslateNumberofPixels)

UpdateoverlayoffsetDisplay( )

Case eManualMoves.RotatingCCWOverlay.overlayAngle = overlay. OverlayAngle + $\odot .1$

If Overlay.overlayAngle $>=36 \odot$ Then Overlay.overlayAngle $=\odot$

Overlay.MoveDeltaPixels $(\Theta, 0)$

UpdateoverlayoffsetDisplay( )

Case eManualMoves.RotatingCWOverlay.OverlayAngle $=$ Overlay. OverlayAngle -0.1 If Overlay.overlayAngle $<=\odot$ Then Overlay. OverlayAngle $=360$

overlay. MoveDeltaPixels $(\odot, \odot)$

UpdateoverlayoffsetDisplay( )

End Select

End Sub 
Private Sub RotatecCWButton_MouseDown(ByVal sender As object, ByVal e As System.

Windows.Forms.MouseEventArgs) Handles RotatecCWButton. MouseDown

ManualMove $=$ eManualMoves. RotatingCCW

End Sub

Private Sub RotateCCWButton_MouseUp(ByVal sender As object, ByVal e As System. Windows.

Forms.MouseEventArgs) Handles RotateCCWButton. MouseUpManualMove $=$ eManualMoves.Stopped

End Sub

Private Sub RotateCWButton_MouseDown(ByVal sender As object, ByVal e As System. Windows .Forms. MouseEventArgs) Handles RotateCWButton. MouseDown ManualMove = eManualMoves. RotatingCW

End Sub 
D: \VideoComparatorProject -

3\VideoComparatorProject \User Interface \VideoComparator . vb

Private Sub RotateCWButton_MouseUp(ByVal sender As object, ByVal e As System. Windows.

Forms.MouseEventArgs) Handles RotateCWButton. MouseUp

ManualMove $=$ eManualMoves.Stopped

End Sub

Private Sub OverlayTintTrackBar_ValueChanged(ByVal sender As object, ByVal e As System.EventArgs) Handles OverlayTintTrackBar.Valuechanged

OverlayColorLabel.BackColor = Color.FromArgb(OverlayTintTrackBar.Value, overlayTintTrackBar.Value, overlayTintTrackBar.Value)

overlay. OverlayColor = OverlayTintTrackBar.Value

End Sub

Private Sub ModePanelHideButton_Click(ByVal sender As System.Object, ByVal e As System

.EventArgs) Handles ModePanelHideButton.Click

ToDoGroupBox.Visible $=$ False

ModeSelectionToolstripMenuItem. Checked = False

End Sub

Private Sub ResetMILDisplay_Click(ByVal sender As System.object, ByVal e As System.

EventArgs) Handles ResetMILDisplay.Click

MILImage.Free( )

MILImage. Allocate( )

End Sub

End Class 
D: \VideoComparatorProject-3\VideoComparatorProject \app.config

$<$ ?xml version="1.๑" encoding="utf-8" ?>

$<$ configuration>

$<$ configsections>

$<$ sectionGroup name="usersettings"

type="System. Configuration. UserSettingsGroup,

System, Version=2.๑.๑.๑, Culture=neutral, PublickeyToken=b77a5c561934e๑89" >

<section name="WindowsApplication1. My.MySettings" type="System. Configuration . ClientSettingssection, System, Version=2.๑.๑.๑, Culture=neutral, PublickeyToken=

b77a5c561934e089" allowExeDefinition="MachineToLocalUser"

requirePermission="false" />

$</$ sectiongroup $>$

$</$ configsections $>$

<system.diagnostics>

<sources>

$<$ !--This section defines the logging configuration for My.Application. Log -->

<source name="DefaultSource" switchName="DefaultSwitch">

$<$ listeners $>$

$<$ add name="FileLog"/>

$<$ !--Uncomment the below section to write to the Application Event Log

$-->$

$<$ ! - -<add name="EventLog" / - - >

$</$ listeners $>$

$</$ source $>$

$</$ sources $>$

$<$ switches>

$<$ add name="DefaultSwitch" value="Information" />

$</$ switches $>$

<sharedListeners>

$<$ add name="FileLog"

type="Microsoft. VisualBasic. Logging. FileLogTraceListener, Microsoft.

VisualBasic, Version=8.0.0.๑, Culture=neutral,

PublickeyToken=b03f5f7f11d50a3a,

processorArchitecture=MSIL"

initializeData="FileLogWriter"/>

$<$ !--Uncomment the below section and replace APPLICATION_NAME with the name

ofyour application to write to the Application Event Log -->

$<!--<$ add name="EventLog" type="System.Diagnostics. EventLogTraceListener"

initializeData="APPLICATION_NAME"/> --> 


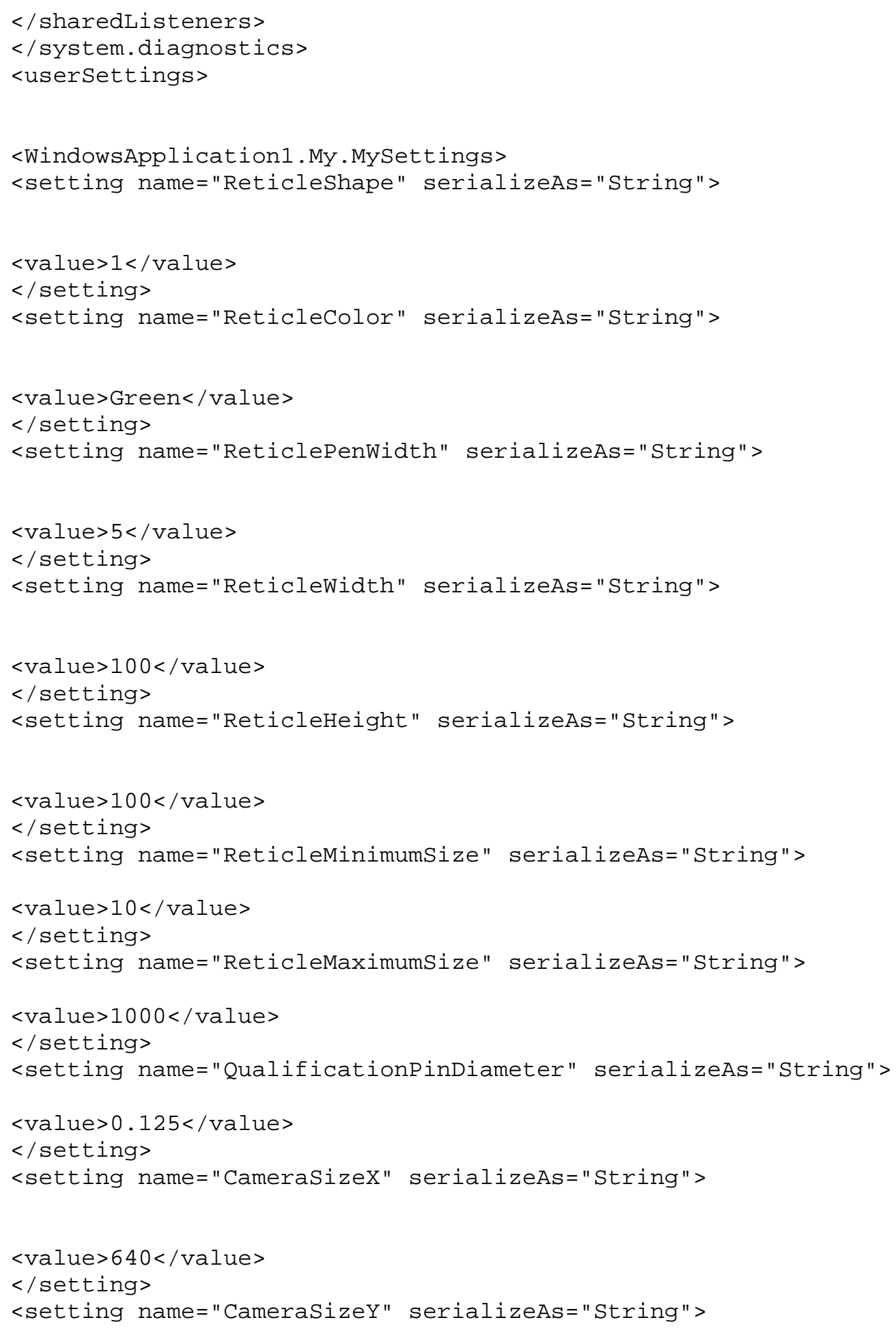




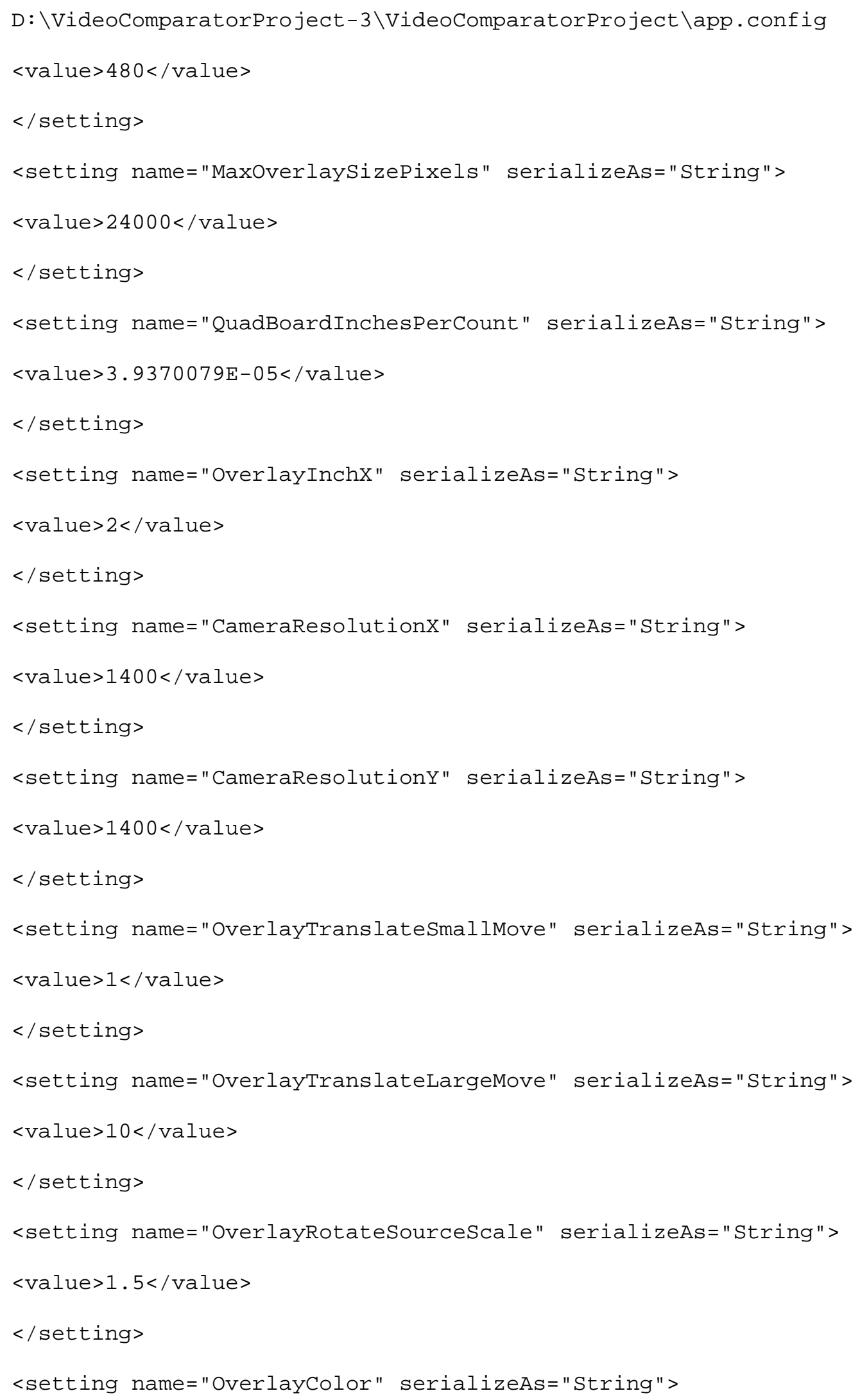


$<$ value $>125<$ /value $>$

$</$ setting $>$

$</$ WindowsApplication1. My. Mysettings $>$

$</$ usersettings $>$

$</$ configuration $>$ 
D: \VideoComparatorProject-3\VideoComparatorProject \Settings. vb

Namespace My

'This class allows you to handle specific events on the settings class:

' The settingchanging event is raised before a setting's value is changed.

' The Propertychanged event is raised after a setting's value is changed.

' The SettingsLoaded event is raised after the setting values are loaded.

' The SettingsSaving event is raised before the setting values are saved.

Partial Friend NotInheritable Class MySettingsEnd Class

End Namespace 
D: \VideoComparatorProject -

3\VideoComparatorProject \WorkingClasses \clsstepper . vb

Public Class clsstepper

Public Sub New()

End Sub

End Class 
D: \VideoComparatorProject -

$3 \backslash$ VideoComparatorProject \WorkingClasses $\backslash c l s R e t i c l e . v b$

Imports System.Windows. Forms

Imports System. Windows

Imports system. Drawing

Public Class clsReticle

Public Enum enumReticleshape

Square

Circle

End Enum

Private_ReticlePen As Pen

Private_ReticleBoundary As Rectangle

Private _ReticlePinDiameter As Double

Private _ReticleCenterPoint As Point

Private_Reticleshape As enumReticleshape

Private_ReticleCanvasHandle As IntPtr

Private_ReticleGraphic As Graphics

Public Sub New()

_ReticlePen $=$ New Pen $($ Color. AntiqueWhite $)$

End Sub

Public Sub Draw()

Select Case_ReticleShape

Case enumReticleshape.Circle

_ReticleGraphic.DrawArc(_ReticlePen,_ReticleBoundary, ๑, 360)

Case enumReticleshape. Square

_ReticleGraphic.DrawRectangle(_ReticlePen,_ReticleBoundary)

End Select

End Sub

Public Property Reticlecanvas() As IntPtr

Get

Return_ReticleCanvasHandle

End Get

Set(Byval value As IntPtr)

_ReticleCanvasHandle = value

_ReticleGraphic = Graphics.FromHwnd(_ReticleCanvasHandle) 


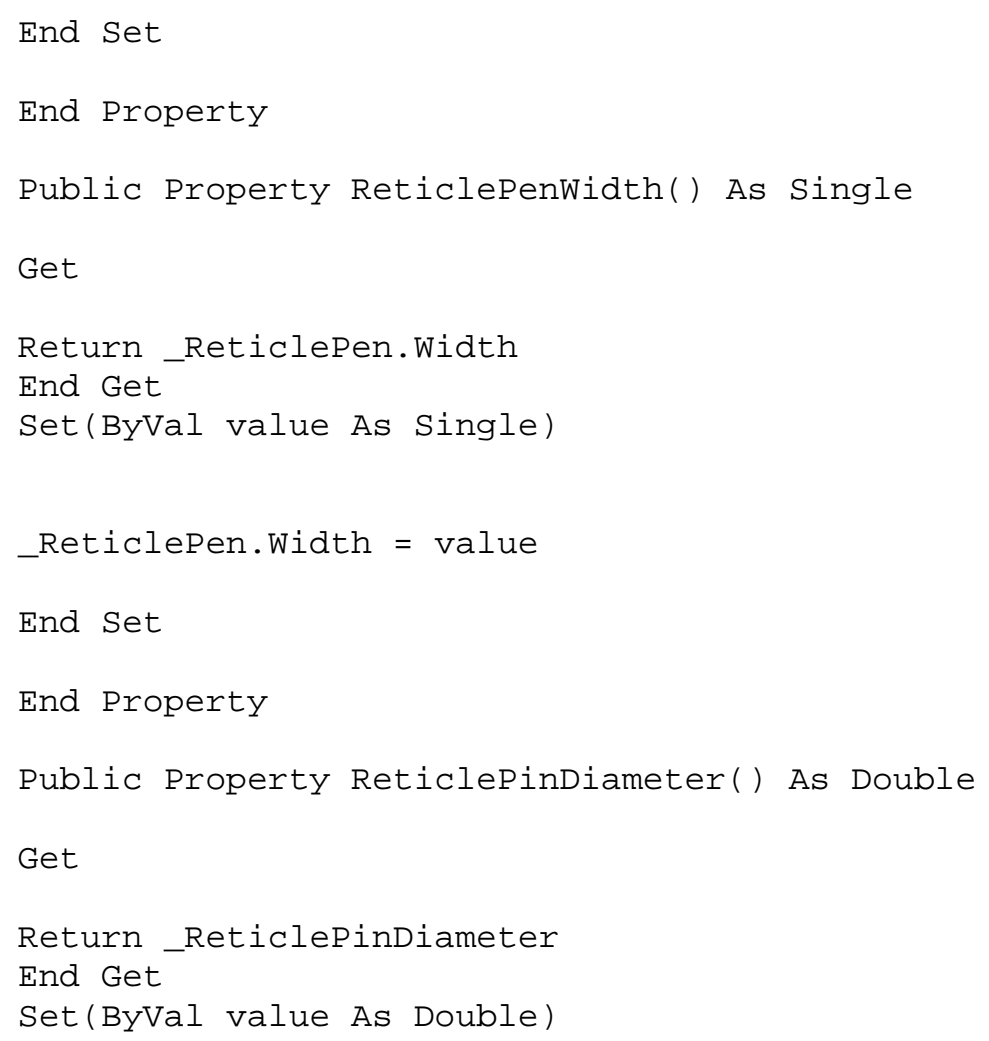


D: \VideoComparatorProject -

3\VideoComparatorProject \WorkingClasses \clsReticle.vb

_ReticlePinDiameter $=$ value

End Set

End Property

Public Property Reticlecolor() As Color

Get

Return_ReticlePen.Color

End Get

Set(Byval value As Color)

_ReticlePen.Color = value

End Set

End Property

Public Property Reticleshape() As enumReticleshape

Get

Return_Reticleshape

End Get

Set(ByVal value As enumReticleshape)

_Reticleshape $=$ value

End Set

End Property

Public Property Reticlewidth() As Integer

Get

Return_ReticleBoundary.Width

End Get

Set(ByVal value As Integer)

_ReticleBoundary. Width = value

_ReticleBoundary.X = _ReticleCenterPoint.X - _ReticleBoundary.Width / 2

End Set

End Property

Public Property ReticleHeight() As Integer 


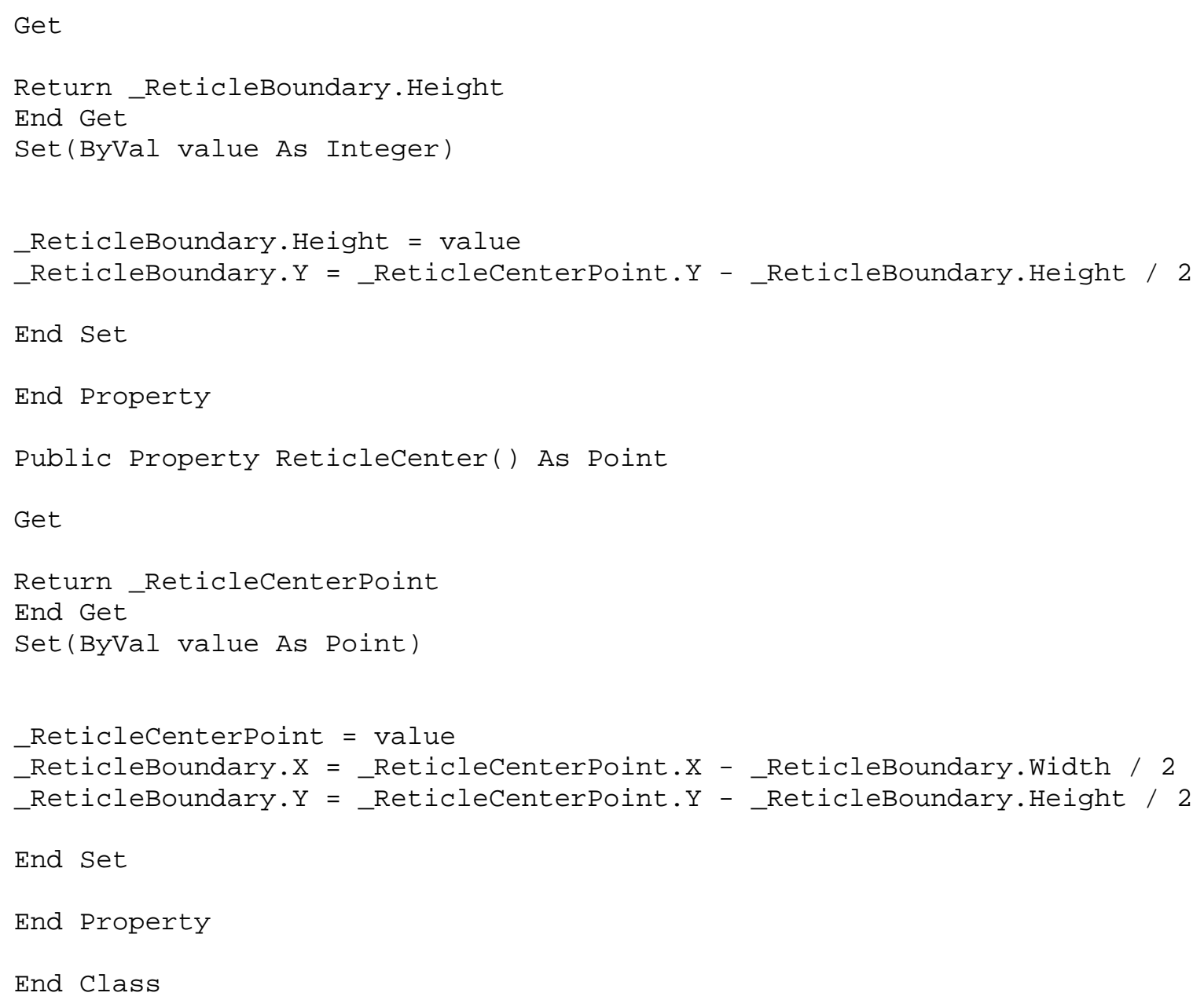


D: \VideoComparatorProject -

3\VideoComparatorProject \WorkingClasses \clsQuadBoard.vb

Imports System. Windows. Forms

Imports System.Timers

Public Class clsQuadBoard

Private _Counter1PreviousCount As Integer

Private _Counter2Previouscount As Integer

Private _Counter1Count As Integer

Private_Counter2Count As Integer

Private _CountersInitialized As Boolean

Private _EncoderInchesPerPulseX As Double

Private EncoderInchesPerPulseY As Double

Private _EncoderPulsesPerInchX As Double

Private _EncoderPulsesPerInchy As Double

Private _EncoderPixelsPerPulseX As Double

Private EncoderPixelsPerPulseY As Double

Private EncoderPulsesPerPixelX As Double

Private EncoderPulsesPerPixely As Double

Public Event Counter1Changed()

Public Event Counter2Changed()

Private WithEvents EncoderTimer As New System.Timers.Timer

Private DaqBoard As MccDaq. MccBoard

Private DaqError As MccDaq.ErrorInfo

Private _CounterWrapPreventer As Integer

Public Sub New()

DaqBoard $=$ New MccDaq $\cdot \operatorname{MccBoard}(\odot)$

DaqError = DaqBoard.C7266Config(1, MccDaq.Quadrature.X4Quad, MccDaq. CountingMode.

NormalMode, MccDaq.DataEncoding.BinaryEncoding, MccDaq. IndexMode.IndexDisabled, MccDaq.Optionstate.Disabled, MccDaq. FlagPins.CarryBorrow, MccDaq.Optionstate.Disabled)

DaqError $=$ DaqBoard.C7266Config(2, MccDaq.Quadrature.X4Quad, MccDaq. CountingMode.

NormalMode, MccDaq.DataEncoding.BinaryEncoding, MccDaq. IndexMode. IndexDisabled, MccDaq.Optionstate.Disabled, MccDaq.FlagPins.CarryBorrow, MccDaq.Optionstate.Disabled)

Counter1PreviousCount $=0$

_Counter2PreviousCount $=0$

EncoderTimer. Interval $=100$

EncoderTimer. Enabled = True

End Sub

Public Readonly Property Counter1Inches() As Double 
Get

Return CDbl(_Counter1Count * EncoderInchesPerPulseY)

End Get

End Property

Public Readonly Property Counter2Inches() As Double Get

Return CDbl(_Counter2Count * EncoderInchesPerPulseX)

End Get

End Property

Public Property EncoderInchesPerPulsex() As Double Get

Return EncoderInchesPerPulseX

End Get

Set(ByVal value As Double)

EncoderInchesPerPulsex = value

EncoderPulsesPerInchX $=1.0 /$ value

End Set

End Property

Public Property EncoderInchesPerPulsey() As Double Get 
D: \VideoComparatorProject -

3\VideoComparatorProject \WorkingClasses \clsQuadBoard.vb

Return_EncoderInchesPerPulseY

End Get

Set(ByVal value As Double)

_EncoderInchesPerPulsey = value

_EncoderPulsesPerInchY $=1.0 /$ value

End Set

End Property

Public Property EncoderPixelsPerPulsex() As Double

Get

Return_EncoderPixelsPerPulsex

End Get

Set(ByVal value As Double)

_EncoderPixelsPerPulsex = value

_EncoderPulsesPerPixelX $=1.0 /$ value

End Set

End Property

Public Property EncoderPixelsPerPulsey() As Double

Get

Return EncoderPixelsPerPulsey

End Get

Set(ByVal value As Double)

_EncoderPixelsPerPulseY = value

_EncoderPulsesPerPixelY $=1.0 /$ value

End Set

End Property

Public Readonly Property CountersInitialized() As Boolean Get

Return_CountersInitialized

End Get 


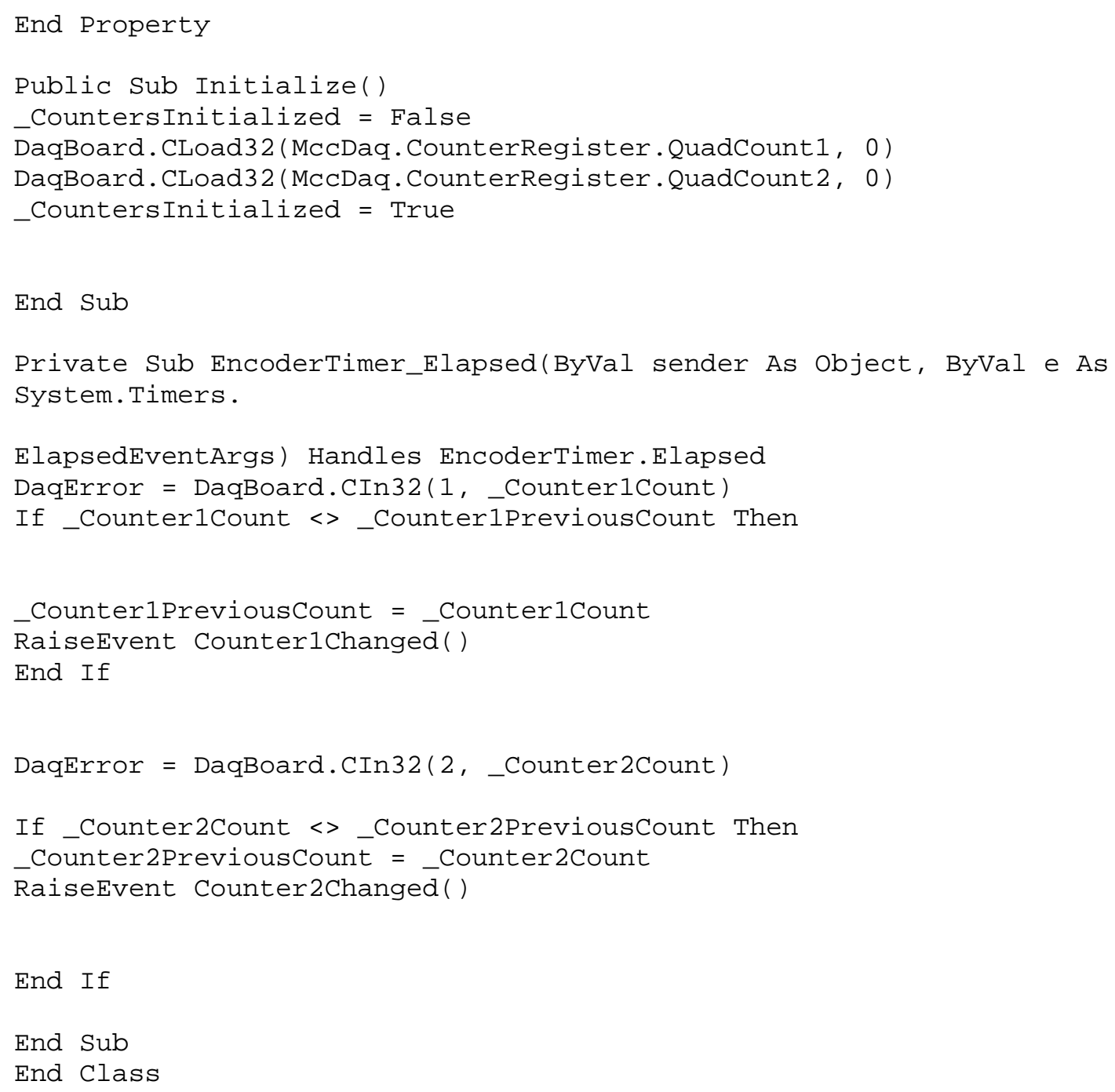


D: \VideoComparatorProject -

$3 \backslash$ VideoComparatorProject \WorkingClasses \clsoverlay. vb

Imports System.Windows. Forms

Public Class clsoverlay

Private _overlayAngleDegrees As Double

Private _overlayAngleRadians As Double

Private _overlayPath As String

Private _ImageXPixels As Integer

Private _ImageYPixels As Integer

Private _ImageDPIX As Double

Private _ImageDPIY As Double

Private _overlayXPixels As Integer

Private _overlayYPixels As Integer

Private _overlayXInches As Double

Private _overlayYInches As Double

Private _overlayDPIX As Double

Private _overlayDPIY As Double

Private _overlayToImagescaleX As Double

Private _overlayToImagescaleY As Double

Private _ImageTooverlayscaleX As Double

Private _ImageTooverlayscaley As Double

Private _overlaySourceRectangle As Rectangle

Private _overlayRotateRectangle As Rectangle

Private _overlayRotateSourcescale As Double

Private _overlayRotateSourceXBorder As Integer

Private _overlayRotateSourceYBorder As Integer

Private _overlaysourceLowerLimit As Point

Private_overlaysourceUpperLimit As Point

Private _overlaysourcecenter As Point

Private _overlaysourceoffsetX As Double

Private _overlaysourceoffsety As Double

Private _overlayCurrentXInches As Double

Private _overlaycurrentYInches As Double

Private _overlayColor As Integer

Private _DeltaxMove As Double

Private _DeltaYMove As Double

Private _DeltaXAdjusted As Double

Private _DeltaYAdjusted As Double

Public Sub New()

End Sub

Public Property Overlaycolor() As Integer

Get

Return_overlaycolor

End Get

Set(ByVal value As Integer) 
_overlayColor $=$ value

End Set

End Property

Public Property OverlayPath() As String

Get

Return _overlayPath

End Get

Set(ByVal value As String)

If Not IsNothing(value) Then

_overlayPath = value

End If

End Set

End Property 


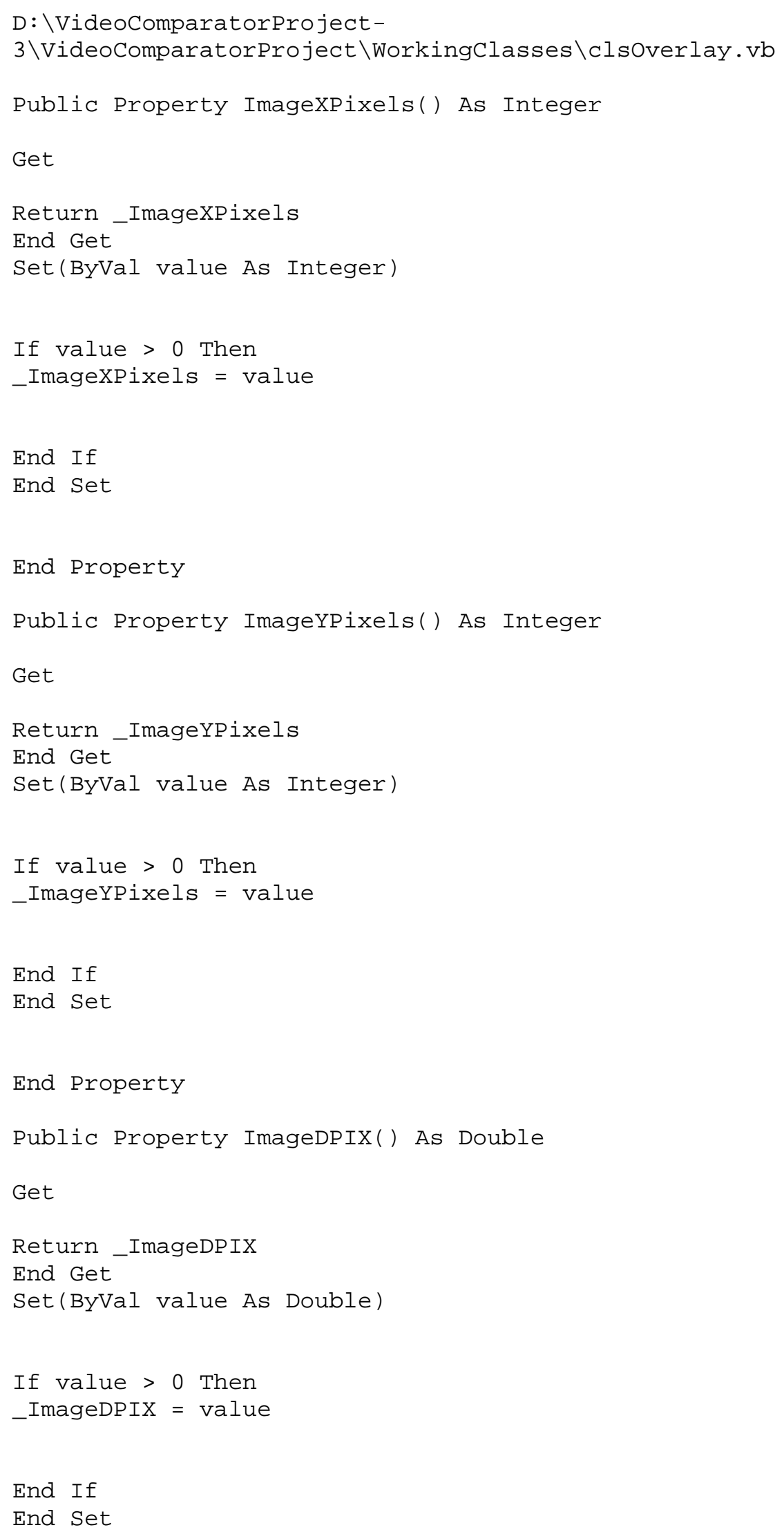




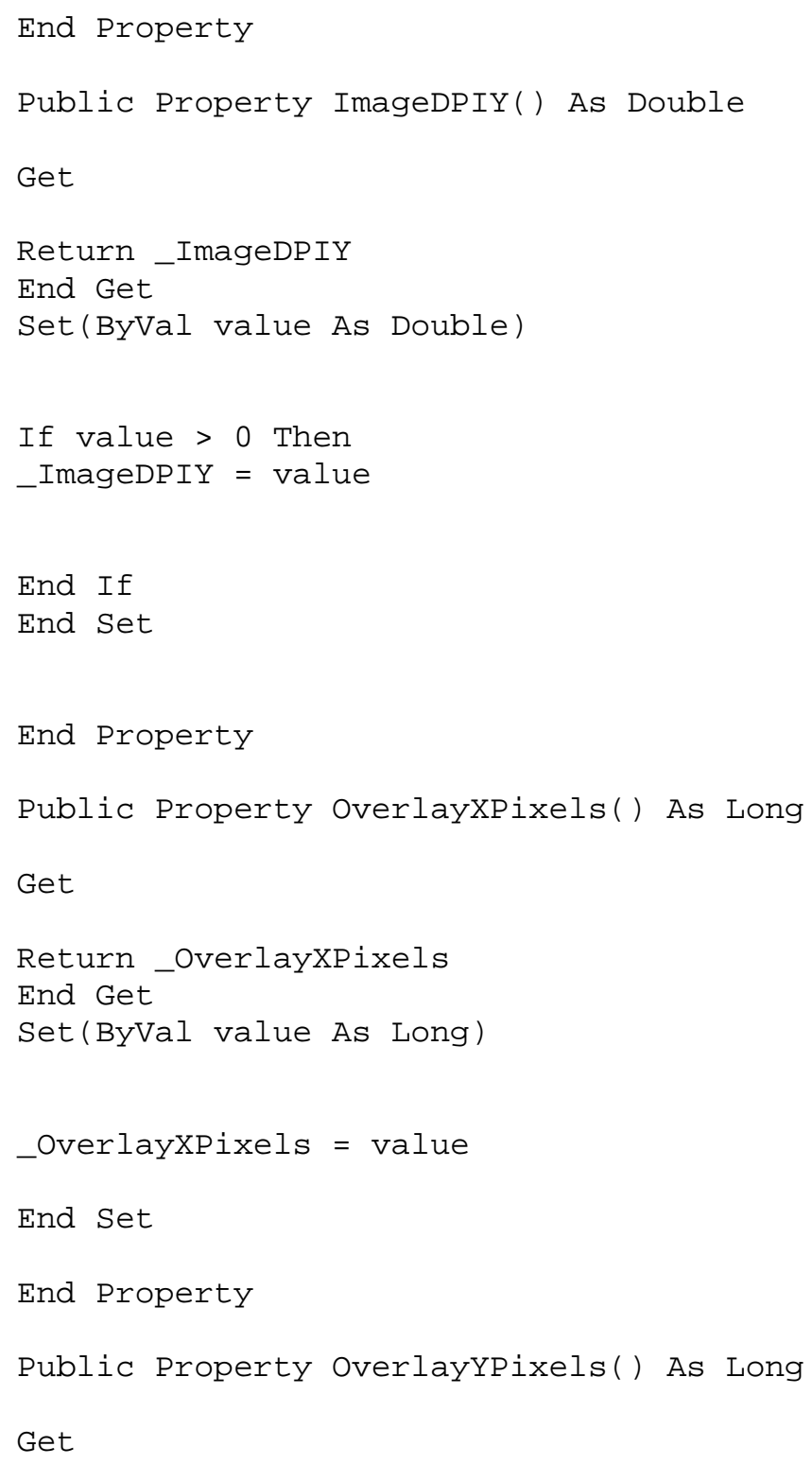


D: \VideoComparatorProject -

3\VideoComparatorProject \WorkingClasses \clsoverlay. vb

Return _overlayYPixels

End Get

Set (ByVal value As Long)

_overlayYPixels = value

End Set

End Property

Public Property OverlayXInches() As Double

Get

Return _overlayXInches

End Get

Set(ByVal value As Double)

_OverlayXInches $=$ value

End Set

End Property

Public Property OverlayYInches() As Double

Get

Return _overlayYInches

End Get

Set(ByVal value As Double)

_overlayYInches $=$ value

End Set

End Property

Public Property OverlayDPIX() As Double

Get

Return_overlayDPIX

End Get

Set(ByVal value As Double)

If value $>\odot$ Then

_overlayDPIX = value 


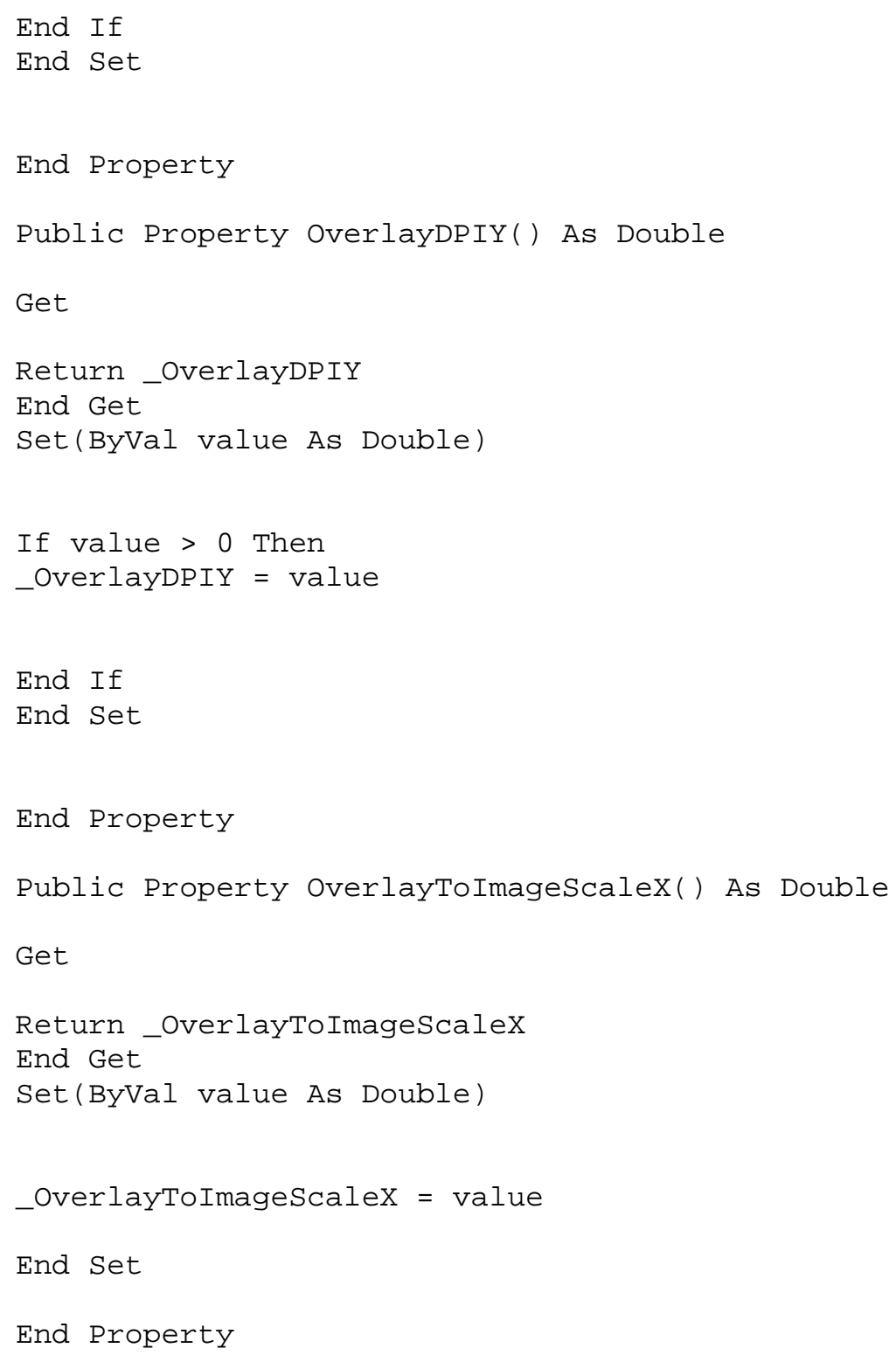


D: \VideoComparatorProject -

3\VideoComparatorProject \WorkingClasses \clsoverlay. vb

Public Property OverlayToImagescaleY() As Double

Get

Return_overlayToImageScaleY

End Get

Set(ByVal value As Double)

_overlayToImageScaleY = value

End Set

End Property

Public Property ImageTooverlayscalex() As Double

Get

Return_ImageTooverlayscaleX

End Get

Set(ByVal value As Double)

_ImageTooverlayscalex = value

End Set

End Property

Public Property ImageTooverlayscaley() As Double

Get

Return_ImageTooverlayscaleY

End Get

Set(ByVal value As Double)

_ImageTooverlayScaleY = value

End Set

End Property

Public Property OverlayAngle() As Double

Get

Return _overlayAngleDegrees

End Get

Set(ByVal value As Double) 


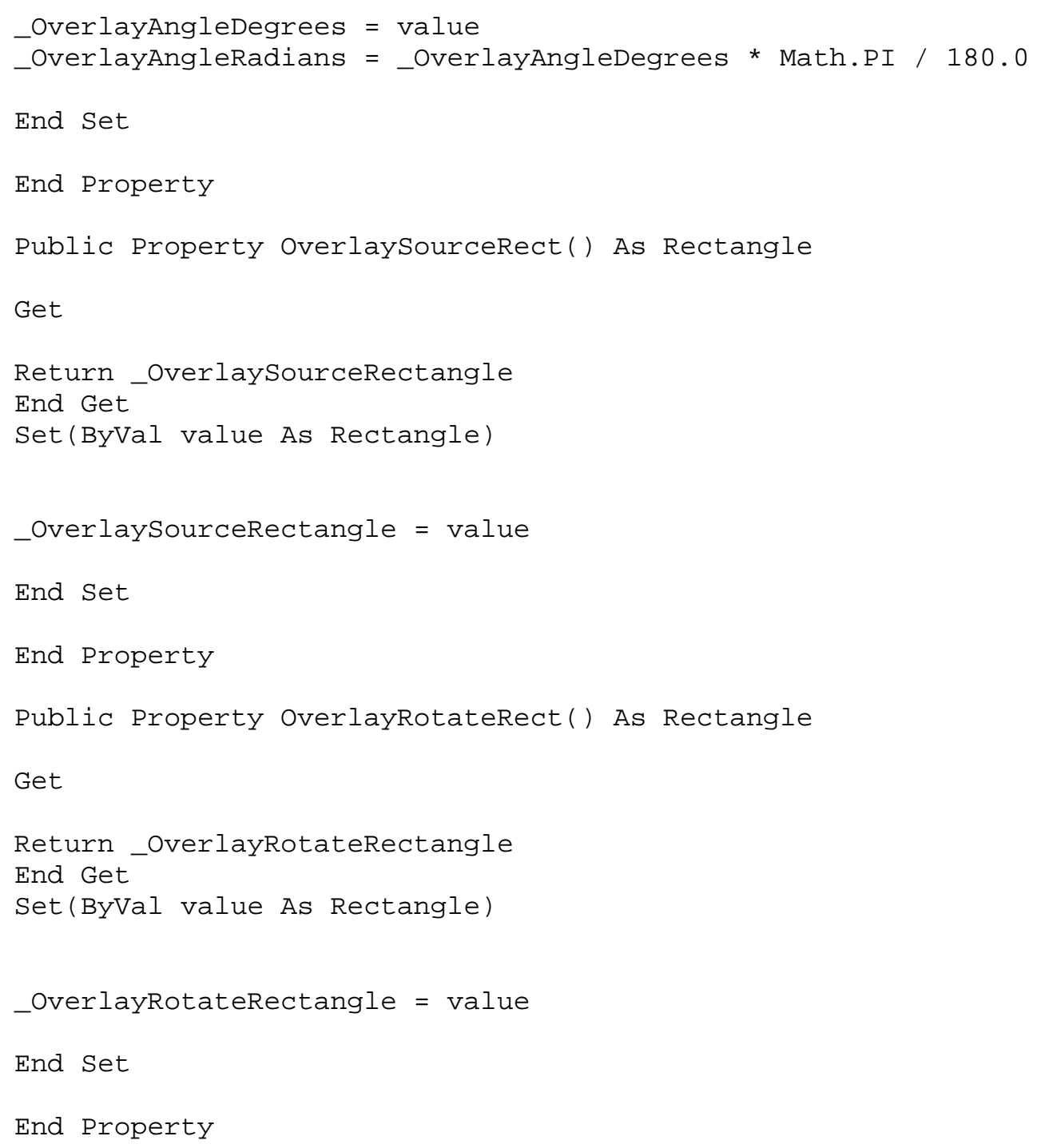


D: \VideoComparatorProject-

3\VideoComparatorProject \WorkingClasses \clsoverlay. vb

Public Property OverlaySourcecenter() As Point

Get

Return_overlaysourcecenter

End Get

Set(ByVal value As Point)

_overlaySourceCenter $=$ value

End Set

End Property

Public Property OverlaySourceoffsetXInches() As Double

Get

Return_overlaysourceoffsetX

End Get

Set(ByVal value As Double)

_overlaySourceoffset $x$ = value

End Set

End Property

Public Property OverlaySourceoffsetYInches() As Double

Get

Return_overlaysourceoffsetY

End Get

Set(ByVal value As Double)

_overlaysourceoffsetY $=$ value

End Set

End Property

Public Property OverlayRotateSourcescale() As Double

Get

Return_overlayRotateSourcescale

End Get

Set (ByVal value As Double) 


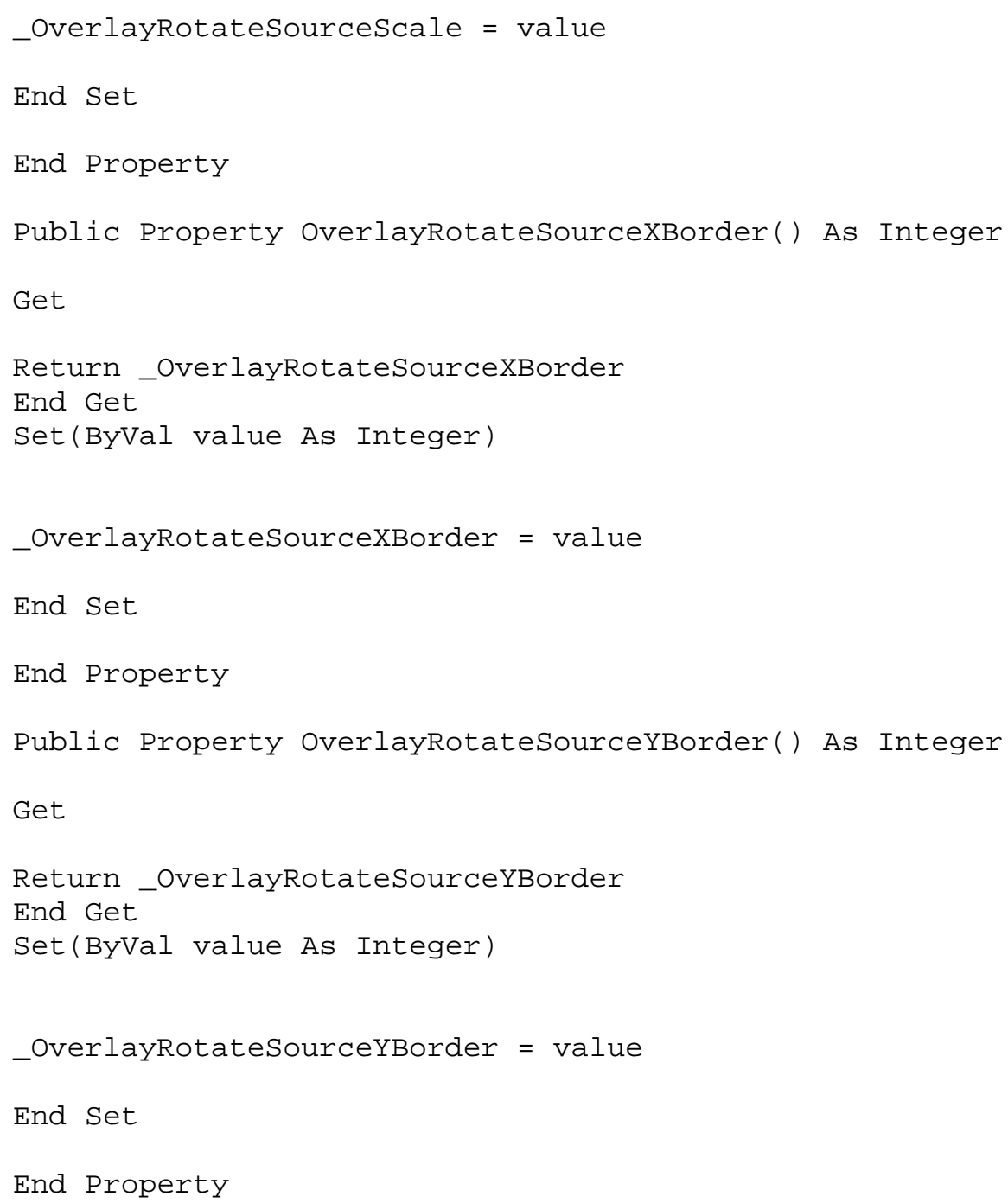


D: \VideoComparatorProject -

3\VideoComparatorProject \WorkingClasses \clsoverlay. vb

Public Sub MoveDeltaPixels(ByVal deltax As Integer, ByVal deltaY As Integer)

_DeltaXMove $=\operatorname{cDbl}(\operatorname{deltaX}) /$ _overlayDPIX

_DeltaYMove $=\operatorname{CDbl}($ deltaY $) /$ _overlayDPIY

${ }_{\star}$ DeltaXAdjusted $=$ _DeltaXMove * Math.Cos(_overlayAngleRadians $)+$ _DeltaYMove

Math.Sin(_overlayAngleRadians)

¿ DeltaYAdjusted = _DeltaYMove * Math.Cos(_overlayAngleRadians) - _DeltaXMove

Math.Sin(_overlayAngleRadians)

_OverlaySourceRectangle.x = _overlaySourceRectangle.X + CInt (_DeltaXAdjusted OverlayDPIX)

If _overlaySourceRectangle.X < _overlaySourceLowerLimit.X Then

_overlaySourceRectangle.X = _overlaySourceLowerLimit.X

If _overlaySourceRectangle. $x>$ _overlaySourceupperLimit. $X$ Then

_overlaySourceRectangle. $X=$ _over laySourceUpperLimit. $X$

‡overlaySourceRectangle.Y = _overlaySourceRectangle.Y - CInt(_DeltaYAdjusted

OverlayDPIY)

If _overlaySourceRectangle.Y < _overlaySourceLowerLimit. Y Then

_overlaySourceRectangle.Y = _overlaySourceLowerLimit.Y

If _overlaysourceRectangle.Y $>$ _overlaySourceUpperLimit. Y Then

_overlaySourceRectangle.Y = _overlaySourceUpperLimit.Y

_overlaysourceoffsetX $=$ _overlaySourceoffsetX $+\operatorname{cDbl}(\operatorname{deltaX}) /$ _overlayDPIX

_overlaysourceoffsetY $=$ _overlaysourceoffsetY $+\operatorname{CDbl}($ deltaY $) /$ _overlayDPIY

_overlayRotateRectangle.x = _overlaySourceRectangle.x -

_overlayRotateSourceXBorder

_OverlayRotateRectangle.Y = _overlaySourceRectangle.Y -

_overlayRotateSourceYBorder

MoveToInches(_overlayCurrentXInches, _overlayCurrentYInches)

End Sub

Public Sub MoveToInches(ByVal ToX As Double, ByVal ToY As Double)

_DeltaXMove $=$ ToX - _overlayCurrentXInches

_DeltaYMove $=$ ToY - _overlayCurrentYInches

${ }_{*}$ DeltaXAdjusted $=$ _DeltaXMove * Math.Cos (_OverlayAngleRadians $)+$ _DeltaYMove

Math.Sin(_overlayAngleRadians)

-DeltaYAdjusted =_DeltaYMove * Math.Cos(_overlayAngleRadians) - _DeltaXMove 


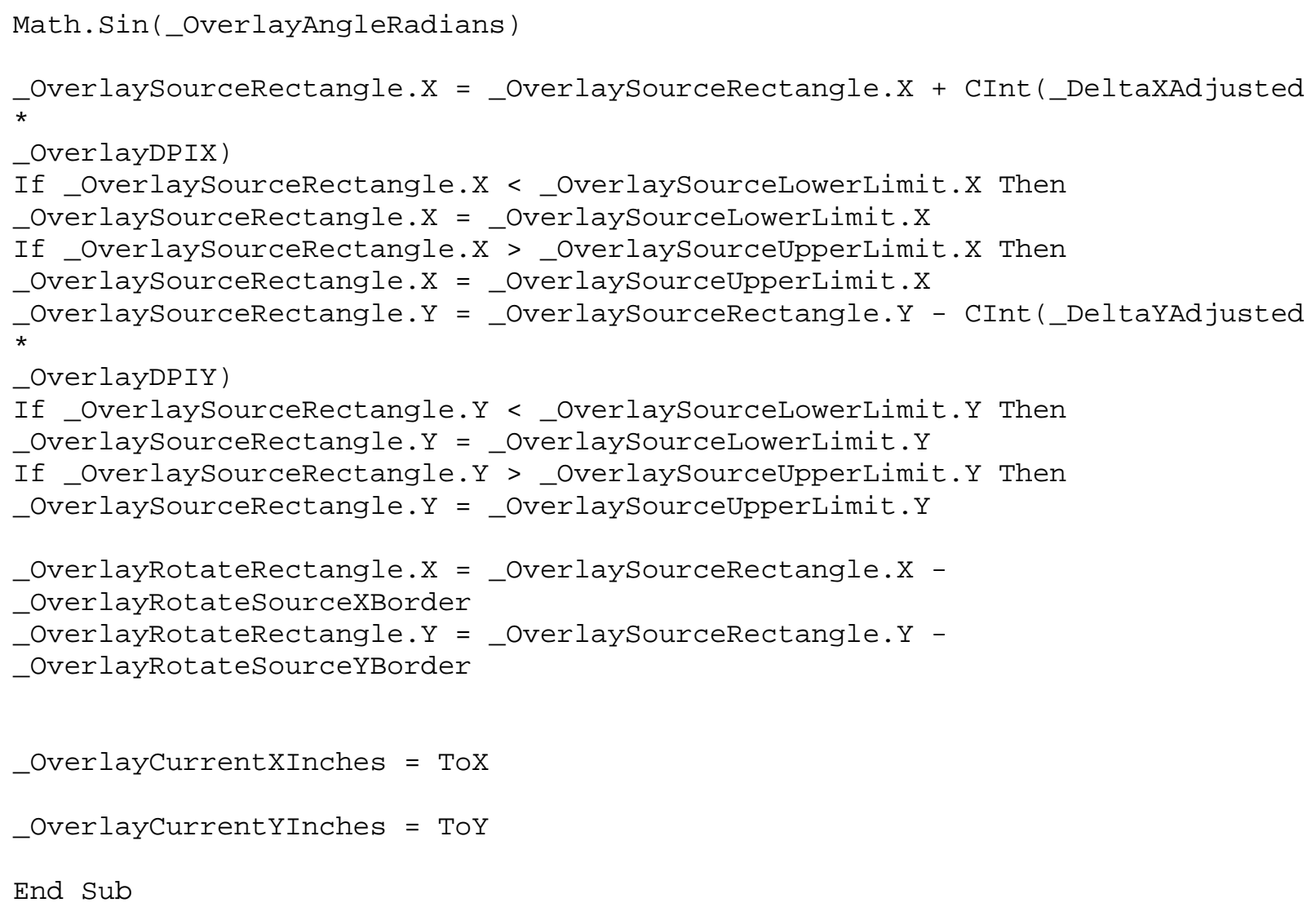


D: \VideoComparatorProject -

$3 \backslash$ VideoComparatorProject \WorkingClasses \clsoverlay. vb

Public Sub UpdateoverlayParameters()

_overlayDPIX = _overlayXPixels / _overlayXInches

_overlayDPIY = _overlayYPixels / _overlayYInches

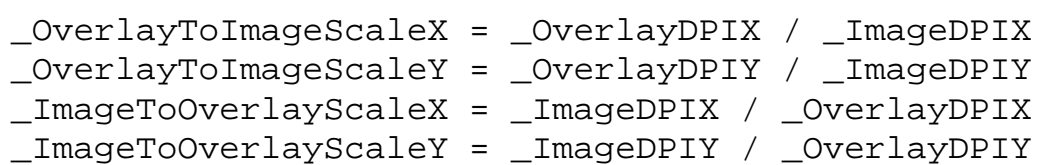

_overlaySourceRectangle.Width = _ImageXPixels *_overlayToImageScaleX

-overlaySourceRectangle.Height = _ImageYPixels * ${ }^{-}$-OverlayToImageScaleY

_overlayRotateRectangle.Width = _overlaySourceRectangle.Width *

OverlayRotateSourcescale

_overlayRotateRectangle.Height = _overlaySourceRectangle. Height *

_overlayRotateSourcescale

_overlayRotateSourceXBorder = (_overlayRotateRectangle.Width -

-OverlaysourceRectangle.Width) $/ 2$

_overlayRotateSourceYBorder = (_overlayRotateRectangle. Height -

_overlaysourceRectangle. Height) / 2

OverlaySourceLowerLimit. $X=0+$ _overlayRotateSourceXBorder +1

-OverlaySourceLowerLimit.Y $=0+$ _overlayRotateSourceYBorder +1

_overlaySourceUpperLimit.X = _overlayXPixels -_overlaySourceRectangle.Width -

OverlayRotateSourceXBorder - 1

_overlaySourceUpperLimit.Y = _overlayYPixels - _overlaySourceRectangle.Height

_overlayRotateSourceYBorder - 1

_overlaySourceRectangle.X = _overlaySourceLowerLimit. $X$

_overlaySourceRectangle.Y = _overlaySourceUpperLimit.Y

_OverlayAngleRadians $=$ _overlayAngleDegrees * Math.PI / 180.0

End Sub

End Class 
D: \VideoComparatorProject -

3\VideoComparatorProject \WorkingClasses \clsCamera.vb

Imports System.Windows. Forms

Public Class clscamera

Private _CameraInitialized As Boolean

Private _GrabbingImages As Boolean

Private _CameraSizePixelsX As Long

Private _CameraSizePixelsY As Long

Private _CameraResolutionX As Double

Private _CameraResolutionY As Double

Private _CameraZoom As Double

Public Sub New()

End Sub

Public Property CameraInitialized() As Boolean

Get

Return_CameraInitialized

End Get

Set(ByVal value As Boolean)

_CameraInitialized $=$ value

End Set

End Property

Public Property CameraSizex() As Long

Get

Return_CameraSizePixelsX

End Get

Set(ByVal value As Long)

_CameraSizePixelsX = value

End Set

End Property

Public Property CameraSizeY() As Long

Get

Return_CameraSizePixelsy

End Get 
Set(ByVal value As Long)

_CameraSizePixelsy = value

End Set

End Property

Public Property CameraResolutionx() As Double

Get

Return_CameraResolutionx

End Get

Set(ByVal value As Double)

_CameraResolutionX = value

End Set

End Property

Public Property CameraResolutionY() As Double

Get

Return_CameraResolutionY

End Get

Set(ByVal value As Double)

CameraResolutionY $=$ value 
D: \VideoComparatorProject -

3\VideoComparatorProject \WorkingClasses \clsCamera.vb

End Set

End Property

Public Property CameraZoom() As Double

Get

Return_CameraZoom

End Get

Set(ByVal value As Double)

_Camerazoom $=$ value

End Set

End Property

End Class 
D: \VideoComparatorProject-3\VideoComparatorProject \Settings. vb

Namespace My

'This class allows you to handle specific events on the settings class:

' The settingchanging event is raised before a setting's value is changed.

' The Propertychanged event is raised after a setting's value is changed.

' The SettingsLoaded event is raised after the setting values are loaded.

' The SettingsSaving event is raised before the setting values are saved.

Partial Friend NotInheritable Class MySettingsEnd Class

End Namespace 